
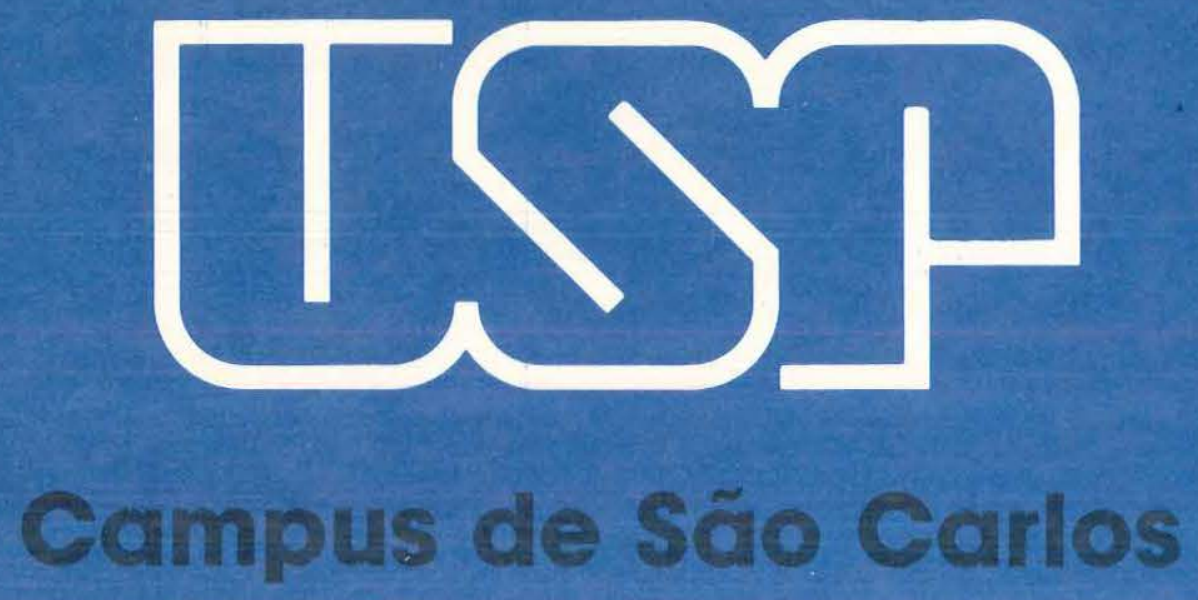

O MÉTODO PRIMAL DUAL BARREIRA LOGARÍTMICA APLICADO AO PROBLEMA DE FLUXO DE CARGA ÓTIMO

Autora: Alessandra Macedo de Souza

Orientador: Prof. Dr. Geraldo Roberto Martins da Costa
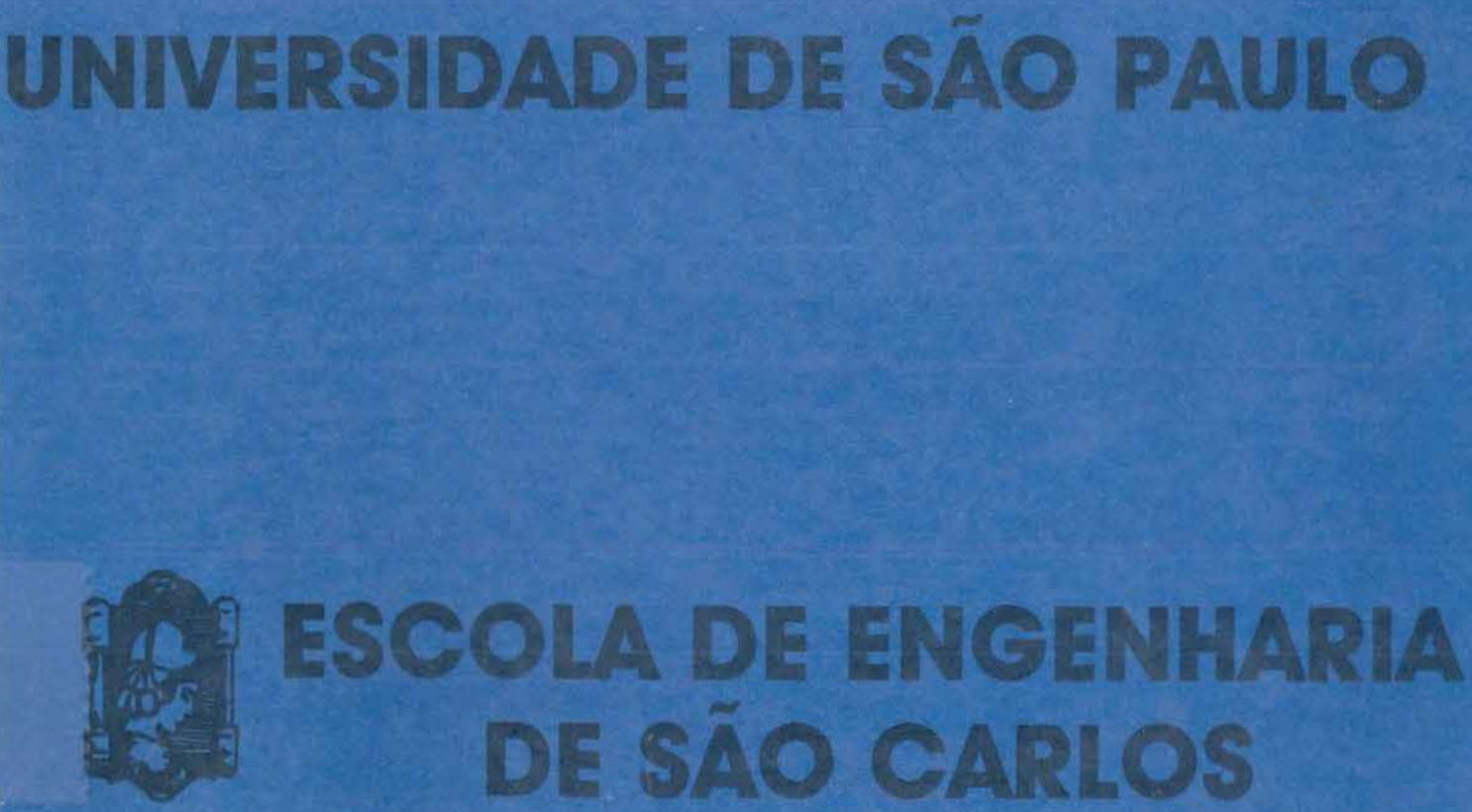


\author{
Universidade de São Paulo \\ Escola de Engenharia de São Carlos \\ Departamento de Engenharia Elétrica
}

\title{
O MÉTODO PRIMAL DUAL BARREIRA LOGARÍTMICA APLICADO AO PROBLEMA DE FLUXO DE CARGA ÓTIMO
}

Alessandra Macedo de Souza

Dissertação apresentada à Escola de Engenharia de São Carlos da Universidade de São Paulo, como parte dos requisito para obtenção do título de Mestre em Engenharia Elétrica.

ORIENTADOR: Prof. Dr. Geraldo Roberto Martins da Costa

São Carlos

1998

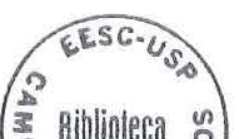




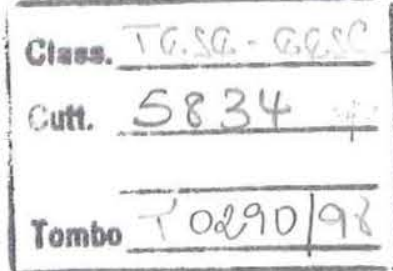

$51 / 5944259$

Ficha catalográfica preparada pela Seção de Tratamento da Informação do Serviço de Biblioteca - EESC-USP

Souza, Alessandra Macedo de

5729m O método primal dual barreira logarítmica aplicado ao problema de fluxo de carga ótimo / Alessandra Macedo de Souza. -- São Carlos, 1998.

Dissertação (Mestrado) -- Escola de Engenharia de São Carlos-Universidade de São Paulo, 1998. Área: Engenharia Elétrica.

Orientador: Prof. Dr. Geraldo Roberto Martins da Costa.

1. Sistemas elétricos de potência. 2. Programação não-linear. 3. Método dos Pontos interiores. 4. Esparsidade. I. Título. 
Candidata: Engenheira ALESSANDRA MACEDO DE SOUZA

Dissertação defendida e aprovada em 18-02-1998 pela Comissão Julgadora:

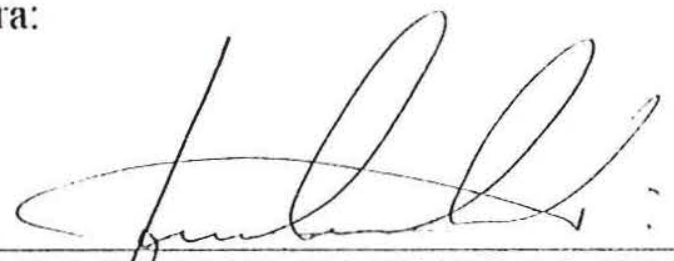

Prof. Doutor GERALDO ROGERTO MAR'TINS DA COSTA (Orientador) (Escola de Engenharia de Sã Carlos - Universidade de São Paulo)

Gaviur

Prof. Doutor CARLOS ÁLBERTO DE CASTRO JUNIOR

(Universidade Estadual de Campinas - UNICAMP)

MPbD

Profa. Doutora CASSWDA MIAIRIA RIBETRO

(Instituto de Ciências Matemáticas de São Carlos - Universidade de São Paulo)

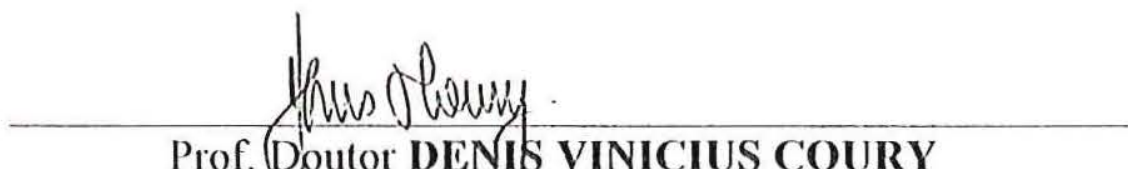

Prof. (Doutor DENIS VINICIUS COURY

Coordenador da Área de Engenharia Elétrica

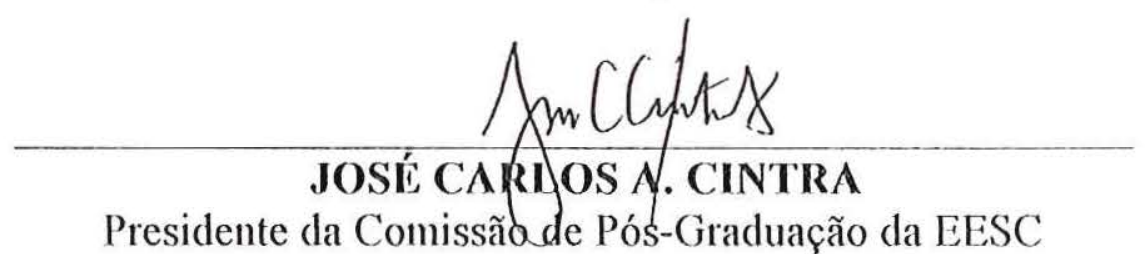


À Deus,

pois Ele é a fonte da vida e inspiração para todo aquele que nEle crê. 
Voz que diz: Clama; e alguém disse: Que hei de clamar? Toda carne é erva, e toda a sua beleza, como as flores do campo. Seca-se a erva, e caem as flores, soprando nelas o hálito do SENHOR. Na verdade o povo é erva. Seca-se a erva e caem as flores, mas a palavra do nosso Deus subsiste eternamente Isaías 40, 6 - 8 


\section{AGRADECIMENTOS}

Ao Professor Dr. Geraldo Roberto Martins da Costa, pela orientação fornecida durante a elaboração deste trabalho.

Aos meus pais, José Trabulo e Graça por todo apoio e incentivo que me deram neste período.

Ao engenheiro e amigo Carlos Costa, pela ajuda na implementação do programa, e pelas valiosas discussões sobre o assunto.

Ao meu irmão Fabrício, as minhas avós Iracilda e Olímpia, aos meus tios e primos, pois reconheço que torceram para a realização deste trabalho.

A todos os professores e funcionários do Departamento de Engenharia Elétrica da EESC - USP - São Carlos pela colaboração.

À Coordenadoria de Aperfeiçoamento de Pessoal de Nível Superior - CAPES, através do programa PICDT, pela bolsa de estudo concedida. 


\section{SUMÁRIO}

LISTA DE FIGURAS ......................................................................... viii

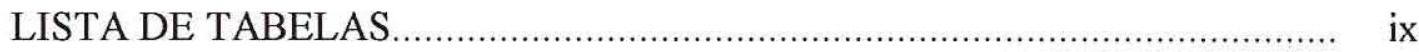

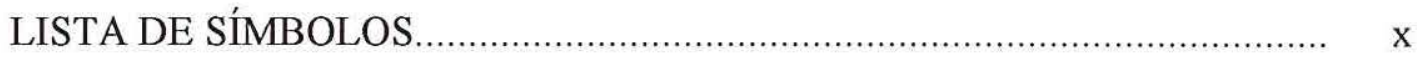

RESUMO

ABSTRACT

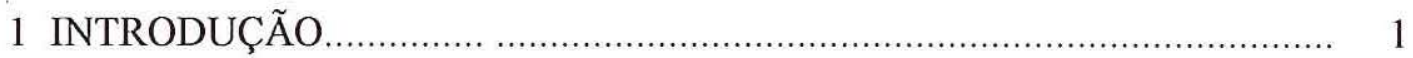

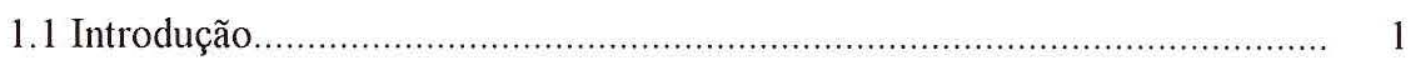

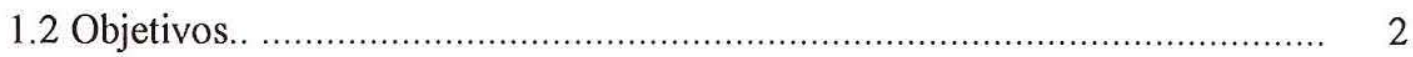

1.3 Organização do Trabalho.........................................................................

2 ESTADO DA ARTE DO PROBLEMA DE FLUXO DE CARGA ÓTIMO.... 4

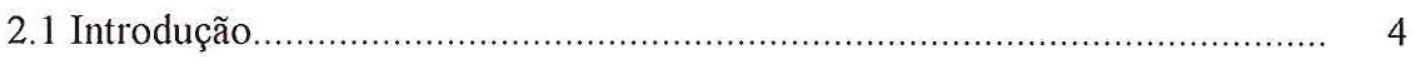

2.2 Histórico

3 O MÉTODO DOS PONTOS INTERIORES............................................... 10

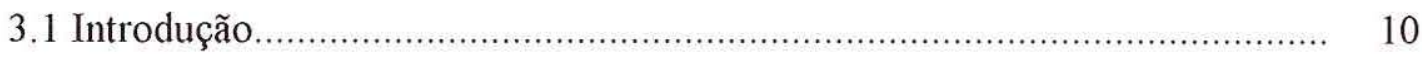

3.2 Método da Função Barreira de Newton Projetada ....................................... 11

3.3 Método Projetivo de Karmarkar …………………………..................... 15

3.4 Relação entre o Algoritmo de Karmarkar e o Método da Barreira de Newton Projetada ........................................................................ 20

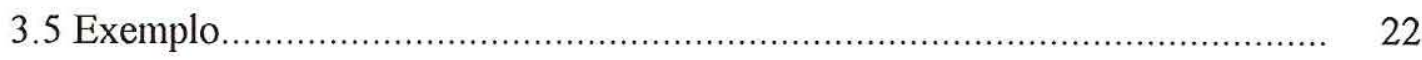


4 FLUXO DE CARGA ÓTIMO UTILIZANDO O MÉTODO DOS PONTOS

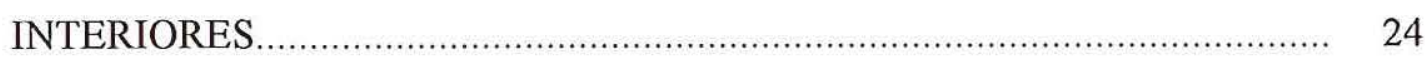

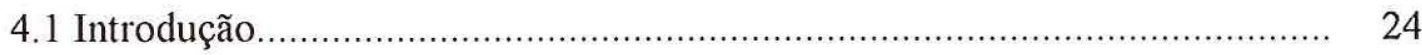

4.2 O Problema de Fluxo de Carga …....................................................... 25

4.3 O Problema de Fluxo de Carga Ótimo ....................................................... 28

4.4 O Método Primal - Dual Barreira - Logarítmica ....................................... 30

4.4.1 Tamanho do Passo ......................................................................... 34

4.4.2 O Parâmetro de Barreira............................................................... 36

4.4.3 Inicialização das Variáveis......................................................... 37

4.5 Algoritmo Simplificado ................................................................ 37

4.6 Implementação Computacional ............................................................ 38

4.7 Rotinas Utilizadas na Solução do Sistema .................................................... 40

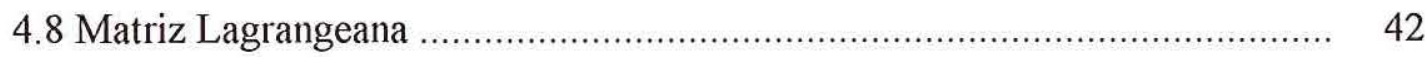

5 TESTES E RESULTADOS ..................................................................... 43

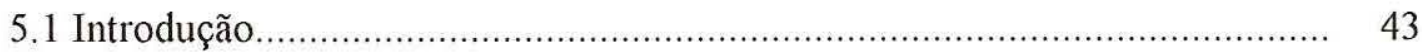

5.2 Sistema de 3 Barras ................................................................................. 43

5.3 Sistema de 14 Barras .......................................................................... 51

5.4 Sistema de 30 Barras ........................................................................... 55

5.5 Sistema de 118 Barras ............................................................................... 59

5.6 Análise entre o fluxo de carga ótimo e o fluxo de carga convencional .......... 62

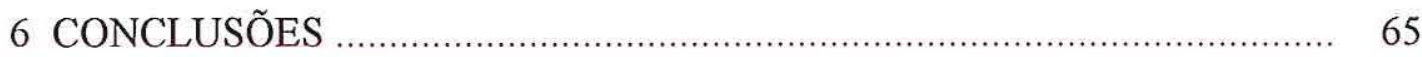

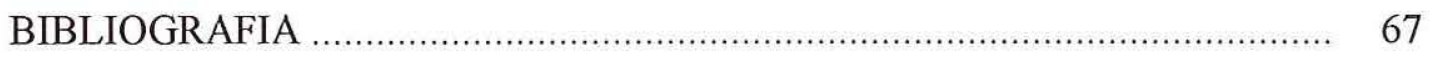

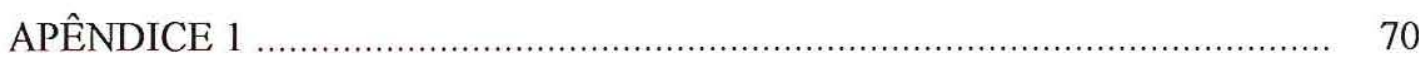

APÊNDICE 2 


\section{LISTA DE FIGURAS}

FIGURA 1 - Ilustração dos métodos pontos interiores e simplex .................... 10

FIGURA 2 - Etapa principal do algoritmo de Karmarkar .............................. 18

FIGURA 3 - Sistema elétrico de 3 barras ......................................................... 44

FIGURA 4 - Função objetivo do sistema de 3 barras ...................................... 50

FIGURA 5 - Convergência de $\mathrm{V}_{1}$ e $\mathrm{V}_{2}$ para o sistema de 3 barras (p.u.) .......... 51

FIGURA 6 - Sistema elétrico de 14 barras ................................................. 52

FIGURA 7 - As restrições $\Delta \mathrm{P}$ e $\Delta \mathrm{Q}$ a cada iteração ......................................... 54

FIGURA 8 - Função objetivo ................................................................. 54

FIGURA 9 - Sistema elétrico de 30 barras ................................................. 56

FIGURA 10 - As restrições $\Delta \mathrm{P}$ e $\Delta \mathrm{Q}$ a cada iteração ........................................... 58

FIGURA 11 - Função objetivo ................................................................... 58

FIGURA 12 - As restrições $\Delta \mathrm{P}$ e $\Delta \mathrm{Q}$ a cada iteração ....................................... 61

FIGURA 13 - Função objetivo ........................................................................... 61 


\section{LISTA DE TABELAS}

TABELA 1 - Resultados dos métodos de Karmarkar e barreira de Newton projetada .............................................................................. 23

TABELA 2 - Sistema exemplo ............................................................... 41

TABELA 3 - Valores das variáveis utilizadas nos cálculos ............................. 46

TABELA 4 - Testes para o sistema de 14 barras........................................... 53

TABELA 5 - Testes para o sistema de 30 barras......................................... 57

TABELA 6 - Sistema de 3 barras (FCO x FCC) ............................................... 63

TABELA 7 - Sistema de 14 barras (FCO x FCC).......................................... 63

TABELA 8 - Sistema de 30 barras (FCO x FCC) ....................................... 63

TABELA 9 - Sistema de 118 barras (FCO x FCC) ...................................... 63

TABELA 10 - Restrições ativas na solução .......................................................... 66 


\section{LISTA DE SÍMBOLOS}

SL - barra de referência

CR - barra de controle de reativo

CG - barra de carga

$\mathrm{A}^{\max }$ - limite máximo da grandeza $\mathrm{A}$

$\mathrm{A}^{\mathrm{min}}$ - limite mínimo da grandeza $\mathrm{A}$

nb - número de barras do sistema

ncr - número de barras de controle de reativo

$\mathrm{V}$ - vetor das magnitudes das tensões

$\theta$ - vetor dos ângulos das tensões

$\mathrm{t}$ - vetor dos taps dos transformadores

s - vetor das variáveis de folga

$\lambda$ - multiplicador de Lagrange para as restrições de igualdade

$\pi$ - multiplicador de Lagrange para as restrições de desigualdade, transformadas em igualdade pelas variáveis de folga

$\mu$ - parâmetro de barreira

$\beta$ - parâmetro utilizado na atualização de $\mu$

$\alpha_{p}$ - tamanho do passo primal

$\alpha_{d}$ - tamanho do passo dual 


\section{RESUMO}

SOUZA, A. M. (1998). O Método Primal-Dual Barreira-Logarítmica Aplicado ao Problema de Fluxo de Carga Ótimo. São Carlos, 1998. 93p. Dissertação (Mestrado) - Escola de Engenharia de São Carlos, Universidade de São Paulo.

Neste trabalho será apresentado um algoritmo de pontos interiores para a solução do problema de fluxo de carga ótimo (FCO). A abordagem proposta é o método primal dual barreira logarítmica. As restrições de desigualdade do problema de FCO são transformadas em igualdades pelo uso de variáveis de folga, e estas são incorporadas na função objetivo através da função barreira logarítmica. A esparsidade da matriz Lagrangeana é explorada e o processo de fatoração é feito por elementos e não por submatrizes. Resultados numéricos de testes realizados em sistemas de 3, 14, 30 e 118 barras serão apresentados com o objetivo de mostrar a eficiência do método.

Palavras-chave: sistemas elétricos de potência, programação não linear, método dos pontos interiores, esparsidade. 


\begin{abstract}
SOUZA, A. M. Optimal Power Flow by a Logarithmic-Barrier Primal-Dual

Method. São Carlos, 1998, 93p. Dissertação (Mestrado) - Escola de

Engenharia de São Carlos, Universidade de São Paulo.
\end{abstract}

In this thesis an interior point algorithm is presented for the solution of the optimal power flow problem (OPF). The approach proposed here is the logarithmicbarrier primal-dual method. The inequality constraints of the optimal power flow problem are transformed into equalities by slack variables that are incorporated into the objective function through the logarithmic barrier function. The sparsity of the Lagrangian matrix is explored and the factorization process is carried out by elements rather than submatrices. Numerical tests results obtained with systems of 3, 14, 30 and 118 buses are presented to show the efficiency of the method.

Keywords: power systems, nonlinear programming, interior point method, sparsity. 


\section{CAPÍTULO 1}

\section{INTRODUÇÃO}

\section{1) INTRODUÇÃO}

As empresas concessionárias de energia elétrica têm por objetivo atender toda a demanda do sistema, fornecendo energia dentro de padrões de qualidade e confiabilidade. Uma ferramenta muito utilizada pelas concessionárias para conhecer o ponto de operação do sistema é o programa de fluxo de carga. Este programa fornece como resultado os valores das tensões e ângulos em todas as barras dos sistemas e taps dos transformadores.

Qualquer mudança que ocorra no sistema de potência, como alterações na geração ou na carga, implicará em uma alteração no ponto de operação do sistema. Um ponto de operação indesejado poderá ser melhorado pelo operador do sistema com base em algum critério local previamente estabelecido. Porém, para sistemas de grandes dimensões, o ajuste das variáveis de controle, utilizando o fluxo de carga convencional, se tornará um processo exaustivo de tentativas e erros, além de não otimizar nenhuma função objetivo. Neste contexto o fluxo de carga ótimo surge como uma ferramenta muito atrativa para a solução das equações do fluxo de carga convencional, ajustando automaticamente e de maneira ótima todas as variáveis de controle do sistema, além de otimizar alguma função objetivo, como por exemplo as perdas de potência ativa na transmissão. 
O fluxo de carga ótimo é um problema de otimização não linear, não convexo e de grande porte, com restrições lineares e não lineares. O problema de FCO foi inicialmente apresentado por CARPENTIER (1962). Desde então muitas pesquisas vêm sendo desenvolvidas com o objetivo de resolvê-lo, como por exemplo os trabalhos de DOMMEL \& TINNEY (1968), SASSON et al. (1973), SUN et al. (1984), GRANVILLE (1994) entre outros. Todas as técnicas propostas tentam resolver o problema de FCO da maneira mais eficiente possível. Devido este problema ser de programação não linear, os algoritmos desenvolvidos para resolvê-lo geralmente exigem que o usuário forneça, de maneira empírica, algum valor para um dado parâmetro da técnica utilizada. No entanto caso ocorra uma escolha errada deste valor, o algoritmo poderá divergir.

\section{2) OBJETIVO}

Este trabalho tem por objetivo apresentar uma aplicação do método dos pontos interiores para a solução do problema de fluxo de carga ótimo. O método de pontos interiores utilizado se chama primal-dual barreira-logarítmica, proposto por GRANVILLE (1994). Esta técnica de otimização procura satisfazer as restrições canalizadas do problema, durante o processo iterativo. Portanto, a convergência pela região factível, característica dos métodos dos pontos interiores, é válida somente para as restrições canalizadas do problema.

A escolha do método primal-dual barreira-logarítmica se deu pelo fato de apresentar rápida convergência, fornecendo uma solução que satisfaz as restrições do problema. A fatoração da matriz Lagrangeana é feita por elementos e não por estruturas em blocos, como utilizado por GRANVILLE (1994). 


\section{3) ORGANIZAÇÃO DO TRABALHO}

No capítulo 1, apresentou-se o problema de fluxo de carga ótimo e definiu-se o objetivo do trabalho.

No capítulo 2, tem-se o estado da arte do problema de fluxo de carga ótimo, onde apresenta-se um breve histórico do problema de fluxo de carga ótimo e também algumas metodologias desenvolvidas na tentativa de resolvê-lo.

No capítulo 3, apresenta-se o método dos pontos interiores inicialmente proposto por KARMARKAR (1984) e também o método da barreira de Newton projetada. O capítulo termina mostrando que sob certas condições ambos os métodos seguem a mesma trajetória.

No capítulo 4, apresenta-se o método primal-dual barreira-logarítmica aplicado ao problema de fluxo de carga ótimo. Discute-se sobre inicialização das variáveis e sobre o mal condicionamento da matriz Lagrangeana.

No capítulo 5, apresentam-se os resultados obtidos com a aplicação da metodologia proposta. Os sistemas elétricos de potência utilizados foram de 3, 14, 30 e 118 barras.

No capítulo 6, apresentam-se as conclusões obtidas com a aplicação deste método e sugestões para futuros trabalhos a serem realizados na área de fluxo de carga ótimo. 


\section{ESTADO DA ARTE DO PROBLEMA DE FLUXO DE CARGA ÓTIMO}

\section{1) INTRODUÇÃO}

O problema de fluxo de carga ótimo foi definido no início da década de 60 . Sua formulação foi inicialmente proposta por CARPENTIER (1962), o qual elaborou o problema de FCO com base no problema de Despacho Econômico, que compreende o quanto cada gerador tem que produzir de potência ativa para atender a demanda do sistema. O FCO é um problema de otimização não-linear, não-convexo e de grande porte, cujo desenvolvimento segue de perto os avanços das técnicas de otimização. Tem por objetivo determinar o ponto de operação ótimo de um Sistema Elétrico de Potência (SEP), otimizando uma função objetivo e satisfazendo as restrições de operação.

Pode ser representado matematicamente por:

$$
\begin{array}{ll}
\min & f(x) \\
\text { s. a } & g(x)=0 \\
& h(x) \leq 0 \\
& 1 \leq x \leq u
\end{array}
$$


onde:

$\mathrm{f}(\mathrm{x})$ é a função objetivo que representa o desempenho do sistema, a qual pode ser o custo de potência ativa gerada, as perdas de potência ativa do sistema, entre outras; $\mathrm{g}(\mathrm{x})$ é o vetor que corresponde às equações do problema de fluxo de carga; $h(x)$ é o vetor que corresponde às inequações funcionais do problema; u e 1 são os limites superiores e inferiores das variáveis do problema; $\mathrm{x}$ é o vetor das variáveis de estado do problema, as quais estão divididas em dois grupos:

- variáveis dependentes:

- tensão em todas as barras de carga do sistema;

- ângulo em todas as barras do sistema;

- variáveis de controle:

- tensão em todas as barras de controle de reativo do sistema;

- potência reativa gerada nas barras de controle de reativo do sistema;

- tap dos transformadores.

A seguir será apresentado um breve histórico de algumas técnicas de otimização já implementadas para a solução do problema (2.1). 


\section{2) HISTÓRICO}

Desde sua formulação inicial na década de 60 , muitos métodos têm sido propostos para resolver o problema de FCO. Alguns deles serão destacados a seguir:

O Método do Gradiente Reduzido, proposto por DOMMEL \& TINNEY (1968), consiste em conhecer a priori um ponto que satisfaça $\mathrm{g}(\mathrm{x})=0$, ou seja, há a necessidade de executar um fluxo de carga antes de dar início ao método. O método consiste em se deslocar de um ponto factível em direção oposta ao gradiente da função objetivo (descida rápida) para um novo ponto também factível, o qual minimiza a função objetivo. Trata as variáveis canalizadas da seguinte maneira: se o limite de um dado parâmetro for alcançado, o mesmo é ajustado para o valor limite que foi violado, conforme abaixo:

$$
x_{i}^{\text {novo }}=\left\{\begin{array}{lll}
u_{i}, & \text { se } & x_{i}^{\text {velho }}+\Delta x_{i}>u_{i} \\
l_{i}, & \text { se } & x_{i}^{\text {velho }}+\Delta x_{i}<1_{i} \\
x_{i}+\Delta x_{i} & \text { caso contrário }
\end{array}\right.
$$

As restrições funcionais de desigualdade são tratadas por parâmetros de penalidade e multiplicadores de Lagrange são usados para associar as equações de fluxo de carga à função objetivo. Se a solução do problema for obtida em uma das duas primeiras condições de (2.2), o componente do vetor gradiente $\partial \mathrm{L} / \partial \mathrm{x}_{\mathrm{i}}$ será diferente de zero. A desvantagem deste método de primeira ordem é a sua lența convergência para alguns tipos de funções, pois as direções de deslocamentos d são ortogonais, isto é, $d_{k+1}^{t} \cdot d=0$, podendo até mesmo resultar em oscilações em torno de um ponto, comprometendo assim a convergência do método. 
O Método da Penalidade, proposto por SASSON et al.(1973), surgiu com o objetivo principal de acelerar a convergência do método utilizado para solução do fluxo de carga ótimo, até então muito lento devido ao fato de ser de primeira ordem. SASSON et al.(1973) propuseram uma técnica de segunda ordem para resolver o problema (2.1), ou seja, utilizar a matriz Hessiana. Esta técnica tem o objetivo de tornar o problema restrito em irrestrito, penalizando todas as restrições de desigualdade que são violadas e as de igualdade que ainda estão longe de zero. A ação da penalidade faz com que o valor da função objetivo aumente quando uma restrição é violada. O algoritmo tentará minimizar a violação e trazer as variáveis de volta para a região factível. Com o aumento do número de iterações estes fatores de penalidade vão aumentando, o que pode causar mal condicionamento da matriz Hessiana, ou seja, elementos muito elevados na diagonal principal, comprometendo a convergência do método.

O Método do Gradiente de Segunda Ordem, proposto por RASHED \& KELLY (1974), tem por objetivo corrigir as deficiências do método proposto por DOMMEL \& TINNEY (1968) através do uso da matriz Hessiana. Este método utiliza multiplicadores de Lagrange para as restrições de igualdade, penalidade para as restrições funcionais de desigualdade e trata as inequações lineares como mostrado em (2.2).

O Método de Newton, proposto por SUN et al.(1984), descreve uma solução para o problema de FCO baseado na formulação de Newton. A cada iteração a função Lagrangeana é aproximada por uma quadrática. O conjunto de restrições de desigualdade é dividido em dois grupos: restrições penalizadas, aquelas introduzidas na função objetivo através de termos de penalidades quadráticos, ou seja, são restrições violadas, e restrições ativas na solução, aquelas que fazem parte do conjunto de restrições de igualdade do problema, ou seja, as equações do fluxo de carga convencional. O ponto ótimo do problema ocorre quando as condições de otimalidade de Karush-Kuhn-Tucker (KKT) estão satisfeitas e as equações do fluxo de carga convencional estão dentro de determinada tolerância. O método apresenta 
convergência de segunda ordem e tem como deficiência a necessidade de identificar as restrições de desigualdade que são ativas no ponto ótimo.

O Método da Lagrangeana Aumentada, proposto por SANTOS et al. (1988), é um método cuja trajetória ocorre pela região infactível do problema. A cada iteração a função Lagrangeana Aumentada é minimizada em relação às variáveis primais utilizando o método de Newton. Os multiplicadores de Lagrange são atualizados visando a maximização da função Lagrangeana Aumentada Dual associada ao problema original. Este método pode ser visto como sendo uma melhora do método proposto por SASSON et al.(1973), pois não há a necessidade de parâmetros de penalidade muito elevados e a matriz Hessiana se torna "bem comportada", ou seja, não há o problema do mal condicionamento da matriz. A solução deste método é obtida quando todas as restrições de igualdade e de desigualdade estão sendo satisfeitas, dentro de uma tolerância especificada.

O Método de Newton Modificado Associado à Função Langrageana Aumentada, proposto por COSTA et al.(1996), tem por objetivo associar os métodos propostos por SUN et al.(1984) e SANTOS et al. (1988). Este método não apresenta a necessidade de identificar as restrições de desigualdade que são ativas na solução, eliminado assim a deficiência do algoritmo de SUN et al. (1984). As restrições de igualdade são tratadas pelo método de Newton, desta forma, estas restrições não serão penalizadas evitando assim o aumento da não linearidade associado ao método da Lagrangeana Aumentada. 
O Método Primal-Dual Barreira-Logarítmica, proposto por GRANVILLE (1994), é um método de pontos interiores que consiste em encontrar o ponto ótimo satisfazendo as restrições canalizadas durante o processo iterativo. Utiliza multiplicadores de Lagrange para as restrições de igualdade e transforma as desigualdades em equações através do uso de variáveis de folga. Estas variáveis são incorporadas na função objetivo através da função barreira logarítmica e penalizadas com o parâmetro de barreira, o qual tenderá a zero com o aumento de iterações. Este algoritmo apresenta muita sensibilidade quanto a escolha do parâmetro de barreira, podendo até mesmo divergir em alguns casos. A solução é encontrada quando todas as restrições do problema original estão satisfeitas. Desataca-se também nesta mesma área de pontos interiores o trabalho de WU et al. (1994), o qual sugere uma extensão do método primal-dual. Seu algoritmo é chamado de método predictor-corrector, e sua diferença em relação ao método primal-dual, é a introdução de termos nãolineares nas condições de otimalidade. Os trabalhos de QUINTANA et al. (1995) e IRISARRI et al. (1997), também contribuem para o avanço das técnicas de otimização que utilizam pontos interiores, visto que esta técnica tem se mostrado muito eficiente para solução de (2.1). 


\section{CAPÍTULO 3}

\section{O MÉTODO DOS PONTOS INTERIORES}

\section{1) INTRODUÇÃO}

O método simplex apresentado por George Dantzig na década de 40, não tinha concorrentes até 1984, quando KARMARKAR (1984) propôs um novo algoritmo para solução de problemas lineares de otimização, cuja filosofia divergia radicalmente do método simplex.

O algoritmo de George Dantzig busca uma solução ótima $x^{*}$, através de deslocamentos entre os pontos extremos da região factível, enquanto que o algoritmo de Karmarkar raramente visita muitos pontos extremos antes de encontrar a solução ótima $x^{*}$. Este método tem por objetivo caminhar pelo interior da região factível, até encontrar o ponto ótimo. Por isso o algoritmo de Karmarkar também é conhecido como o método dos pontos interiores. A figura 1 ilustra a diferença entre as citadas técnicas de otimização.

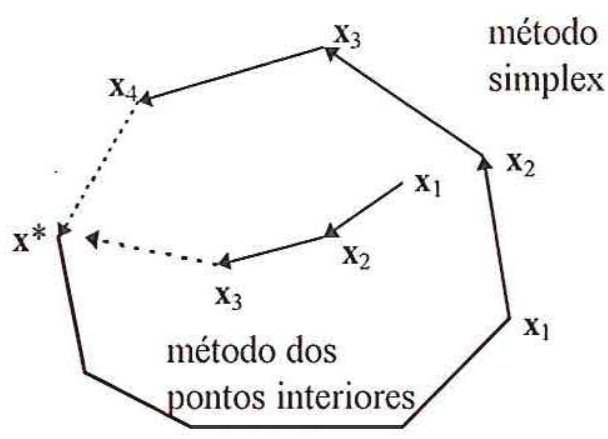

FIGURA 1 - Ilustração dos métodos dos pontos interiores e simplex 
SHERKAT \& IKURA (1994) comprovaram que o método dos pontos interiores é bastante competitivo com o simplex, para problemas de pequeno e médio porte, porém definitivamente superior para problemas de grande dimensão.

Uma grande quantidade de algoritmos diferenciados usando a técnica dos pontos interiores foram elaborados. Como exemplo, podem ser citados os métodos de escala-afim, primal de centros, primais-duais de redução de potencial, entre outros como mencionado em MATUMOTO (1996). Todos estes métodos possuem a preocupação em manter-se longe da fronteira da região factivel. Para isso, uma ferramenta apresentada inicialmente por FRISCH (1955), a função barreira logarítmica, tornou-se muito importante e é definida por:

$$
f(x)=-\sum_{i=1}^{n} \ln x_{i}, x \in \mathfrak{R}^{n}, x>0
$$

GILL et al. (1986) utilizaram esta ferramenta para desenvolver o método da barreira de Newton projetada para solução de problemas lineares de otimização, e também provaram que para determinados tipos de problemas de programação linear e sob certas condições, o seu algoritmo é equivalente ao de Karmarkar.

O método da barreira de Newton projetada, o algoritmo de Karmarkar e a relação entre estes dois métodos, serão apresentados a seguir.

\section{2) MÉTODO DA FUNÇÃO BARREIRA DE NEWTON PROJETADA}

O método da barreira de Newton projetada, tem por objetivo tratar as restrições de desigualdade dos problemas de otimização, através da criação da função barreira, definida em (3.1). 
Considere o seguinte problema de otimização, com função objetivo e restrições lineares:

$$
\begin{array}{ll}
\min & c^{t} x \\
\text { s. } a & A x=b \\
& x \geq 0
\end{array}
$$

onde:

A é uma matriz $\mathrm{m} \mathrm{x} \mathrm{n;}$

b e c são vetores de dimensão $m$ e $n$ respectivamente.

Aplicando a função barreira definida em (3.1) no problema (3.2), o novo problema a ser otimizado é:

$$
\min F(x)=c^{t} x-\mu \sum_{i=1}^{n} \ln x_{i}
$$

s. a $\quad \mathrm{Ax}=\mathrm{b}$

onde:

$\mathrm{F}(\mathrm{x})$ é a função objetivo do novo problema, a qual é uma combinação da função objetivo do problema original e da função barreira penalizada com o parâmetro $\mu$; $\mu(\mu>0)$ é o parâmetro de barreira; $\mathrm{x}$ é um vetor estritamente positivo.

O problema com função objetivo não-linear e restrições lineares, mostrado em (3.3), pode ser resolvido por um método de direções factíveis. No método da barreira de Newton projetada, as variáveis são atualizadas da seguinte forma:

$$
x_{k+1}=x_{k}+\alpha d_{k}
$$


onde:

d é o vetor direção de dimensão n;

$\alpha(\alpha>0)$ é o tamanho do passo.

O vetor de direção d, proposto por GILL et al. (1986), é definido como sendo a solução do problema de programação quadrática mostrado em (3.5), onde a função objetivo é uma aproximação quadrática de $\mathrm{F}(\mathrm{x})$ obtida através da série de Taylor.

$$
\min \nabla F(x) d+\frac{1}{2} d^{t} H(x) d
$$

s. a $\quad \mathrm{Ad}=0$

Com o objetivo de tornar o problema restrito (3.5) em um problema irrestrito, multiplicadores de Lagrange são usados para incorporar as restrições à função objetivo, e desta forma tem-se que a função Lagrangeana é dada por:

$L(d, \lambda)=\nabla F(x) d+\frac{1}{2} d^{t} H(x) d-\lambda(A d)$

onde:

$\lambda$ é o vetor dos multiplicadores de Lagrange associado à restrição de igualdade.

Aplicando as condições de otimalidade em (3.6), tem-se o seguinte sistema de equações:

$$
\begin{aligned}
& \nabla_{\mathrm{d}}=\nabla \mathrm{F}(\mathrm{x})+\mathrm{d} H(\mathrm{x})-\lambda \mathrm{A}=0 \\
& \nabla_{\lambda_{\mathrm{s}}}=-(\mathrm{Ad})=0
\end{aligned}
$$


Reescrevendo (3.7) no formato matricial, tem-se que:

$$
\left(\begin{array}{cc}
H(x) & A^{t} \\
A & 0
\end{array}\right)\left(\begin{array}{c}
-d \\
\lambda
\end{array}\right)=\left(\begin{array}{c}
\nabla F(x) \\
0
\end{array}\right)
$$

$\mathrm{O}$ vetor gradiente e a matriz Hessiana de $\mathrm{F}(\mathrm{x})$ só existem para todo $\mathrm{x}_{\mathrm{i}} \neq 0$. Desde que $\mathrm{H}(\mathrm{x})$ é definida positiva quando $\mathrm{x}>0$, a direção $\mathrm{d}$ de Newton é de descida e única para $\mathrm{F}(\mathrm{x})$, isto é $\nabla \mathrm{F}(\mathrm{x}) \mathrm{d}<0$. O vetor gradiente e a matriz Hessiana de $\mathrm{F}(\mathrm{x})$ são dados por:

$$
\begin{aligned}
& \nabla \mathrm{F}(\mathrm{x})=\mathrm{c}-\mu \mathrm{D}^{-1} \mathrm{e} \\
& \mathrm{H}(\mathrm{x})=\mu \mathrm{D}^{-2}
\end{aligned}
$$

onde:

$\mathrm{D}=\operatorname{diag}\left\{\mathrm{x}_{1}, \mathrm{x}_{2}, \ldots, \mathrm{x}_{\mathrm{n}}\right\}$;

$\mathrm{e}^{\mathrm{t}}=(1,1, \ldots, 1)$

Substituindo (3.9) em (3.8), o sistema pode ser reescrito da seguinte maneira:

$$
\left(\begin{array}{cc}
\mu \mathrm{D}^{-2} & \mathrm{~A}^{\mathrm{t}} \\
\mathrm{A} & 0
\end{array}\right)\left(\begin{array}{c}
-\mathrm{d} \\
\lambda
\end{array}\right)=\left(\begin{array}{c}
\mathrm{c}-\mu \mathrm{D}^{-1} \mathrm{e} \\
0
\end{array}\right)
$$

Inicialmente se escolhe um valor para o parâmetro de barreira, resolve-se o sistema (3.10) e a atualização de $\mu$ ocorre empiricamente. A cada iteração seu valor deve ser reduzido, isto é, deve-se multiplicá-lo por um fator constante e menor que um. Quando $\mu$ tende a zero, a solução ótima do problema (3.3) converge para a solução ótima do problema original (3.2), conforme FIACCO \& McCORMICK (1968). 
O método dos pontos interiores proposto por Karmarkar e a relação que há entre este método e o algoritmo proposto por GILL et al. (1986), serão mostrados a seguir.

\section{3) MÉTODO PROJETIVO DE KARMARKAR}

Como apresentado por BAZARAA et al. (1990), para a aplicação do algoritmo de pontos interiores proposto por Karmarkar, deve-se assumir que o problema linear esteja na seguinte forma:

$\min c^{t} x$

$$
\begin{array}{ll}
\text { s. } & \text { A } x=0 \\
& e^{t} x=1 \\
& x \geq 0
\end{array}
$$

onde:

A é uma matriz $\mathrm{m} \times \mathrm{n}$;

c e A são inteiros;

$e^{t}=(1,1, \ldots, 1)$.

Neste método algumas suposições devem ser satisfeitas.

S.1) O valor inicial $x_{o}^{t}=\left(\frac{1}{n}, \ldots ., \frac{1}{n}\right)$ é factível para o problema (3.11);

S.2) $\sum_{i=1}^{n} x_{i}=1$;

S.3) Seja $x^{*}$ a solução ótima de (3.11) então $c^{t} x^{*}=0$; 
S.4) A soma dos coeficientes das restrições homogêneas deve ser nula, ou seja, $\mathrm{A} \mathrm{e}=0$.

Este método trabalha com transformações de variáveis através das seguintes relações:

- transformação do espaço-x para o espaço-y

$y=\frac{D_{k}^{-1} x}{e^{t} D_{k}^{-1} x}$

- transformação do espaço-y para o espaço-x

$x=\frac{D_{k} y}{e^{t} D_{k} y}$

onde:

$\mathrm{D}_{\mathrm{k}}=\operatorname{diag}\left\{\mathrm{x}_{\mathrm{k} 1}, \ldots, \mathrm{x}_{\mathrm{kn}}\right\}$;

k é a iteração.

Utilizando a transformação (3.13), o problema (3.11) pode ser transformado no seguinte problema no espaço-y:

$$
\begin{array}{ll}
\min & c^{t} \frac{D_{k} y}{e^{t} D_{k} y} \\
\text { s. a } & A D_{k} y=0 \\
& e^{t} y=1 \\
& y \geq 0
\end{array}
$$

Apesar das restrições permanecerem lineares, a função objetivo passou a ser um quociente de funções lineares. Este tipo de problema é conhecido como 
programação linear fracional. Contudo, pela suposição S.3, o valor da função objetivo de (3.14) é nulo no ponto ótimo, e por isso apenas o numerador deve ser minimizado, desde que o denominador tem que ser estritamente maior do que zero. Assim, (3.14) pode ser reescrito da seguinte maneira:

$$
\begin{array}{ll}
\min & \overline{\mathrm{c} y} \\
\text { s.a } & \text { Py }=\mathrm{P}_{\mathrm{o}} \\
& \mathrm{y} \geq 0
\end{array}
$$

onde:

$\overline{\mathrm{c}}=\mathrm{c}^{\mathrm{t}} \mathrm{D}_{\mathrm{k}}$;

$P=\left[\begin{array}{c}A D_{k} \\ e^{t}\end{array}\right]$

$P_{0}=\left[\begin{array}{c}0 \\ e^{t}\end{array}\right]$

Para solucionar o problema (3.15), o algoritmo introduz uma nova restrição, a qual é definida como sendo uma esfera ou circunferência $B\left(y_{0}, r\right)$ de dimensão $n$, com centro em $y_{o}^{t}=(1 / n, \ldots ., 1 / n)$ e de raio r. A interseção desta circunferência com $S_{y}=\left\{y: e^{t} y=1, y \geq 0\right\}$, é uma circunferência de dimensão (n-1) inscrita em $S_{y}$ com o mesmo centro e raio, como mostra a figura 2. 


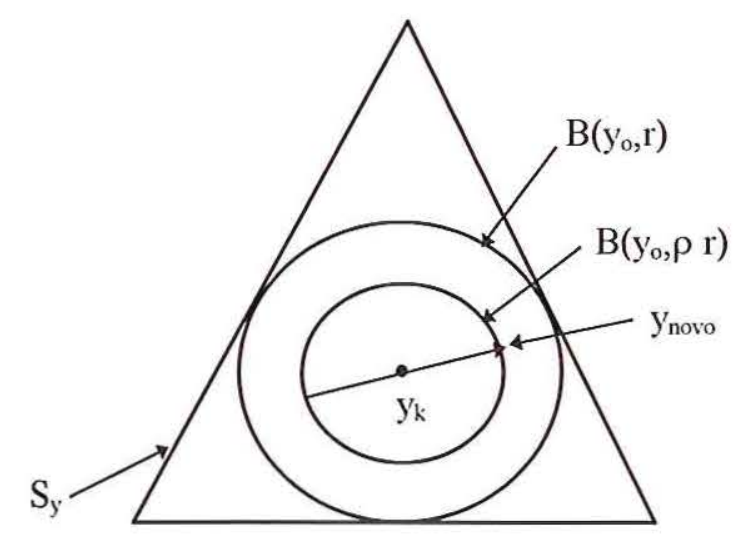

FIGURA 2 - Etapa principal do algoritmo de Karmarkar

O raio $\mathrm{r}$ é a distância do centro $(1 / \mathrm{n}, \ldots \ldots, 1 / \mathrm{n})$ do $\mathrm{S}_{\mathrm{y}}$ para o centro de uma de suas faces, cujas dimensões são (n-1). Assim, considerando a face na qual o componente $\mathrm{y}_{1}$ é nulo, por exemplo, o raio $\mathrm{r}$ será dado pela distância do ponto $(1 / \mathrm{n}, \ldots ., 1 / \mathrm{n})$ ao ponto $(0,1 /(\mathrm{n}-1), \ldots ., 1 /(\mathrm{n}-1)), \operatorname{logo}$ :

$\mathrm{r}=\frac{1}{\sqrt{\mathrm{n}(\mathrm{n}-1)}}$

Definindo $c_{p}$ como a projeção de $\bar{c}^{\mathrm{t}}$, o novo ponto encontrado no espaço-y será dado por:

$y_{\text {novo }}=y_{o}-\rho r \frac{c_{p}}{\left\|c_{p}\right\|}$

onde:

$c_{p}=\left[I-P^{t}\left(P P^{t}\right)^{-1} P\right] \bar{c}^{t}$

$\rho=\frac{(n-1)}{3 n} ; 0<\rho<1$ 
Resumindo, podemos apresentar o algoritmo de Karmarkar da seguinte maneira:

Inicialização:

Calcular $\rho, \mathrm{r}$ e inicializar $\mathrm{x}_{\mathrm{o}}^{\mathrm{t}}=(1 / \mathrm{n}, \ldots \ldots, 1 / \mathrm{n})$. Determinar $\varepsilon$, que será a tolerância desejada.

Passo Principal:

1. Se $c^{t} x<\varepsilon$ então PARE

2. Calcular

$$
\begin{aligned}
& \mathrm{D}_{\mathrm{k}}=\operatorname{diag}\left\{\mathrm{x}_{\left.\mathrm{k} 1, \ldots . ., \mathrm{x}_{\mathrm{kn}}\right\}}\right. \\
& \mathrm{y}_{\mathrm{o}}^{\mathrm{t}}=\left(\frac{1}{\mathrm{n}}, \ldots \ldots, \frac{1}{\mathrm{n}}\right) \\
& \mathrm{P}=\left[\begin{array}{c}
\mathrm{AD}_{\mathrm{k}} \\
\mathrm{e}^{\mathrm{t}}
\end{array}\right] \\
& \overline{\mathrm{c}}=\mathrm{c}^{\mathrm{t}} \mathrm{D}_{\mathrm{k}} \\
& \mathrm{c}_{\mathrm{p}}=\left[\mathrm{I}-\mathrm{P}^{\mathrm{t}}\left(\mathrm{PP}^{\mathrm{t}}\right)^{-1} \mathrm{P}\right] \overline{\mathrm{c}}^{\mathrm{t}}
\end{aligned}
$$

3.Atualizar $\mathrm{y}_{\text {novo }}$ utilizando (3.20)

4.Fazer a transformação do espaço-y para o espaço-x através de (3.13)

5.Retorne ao passo 1 .

Este algoritmo apresenta como desvantagem, as muitas operações matriciais que devem ser implementadas para o cálculo da variável $c_{p}$, mas como será visto no próximo item, o algoritmo proposto por GILL et al. (1986), cuja implementação é mais simples, segue a mesma trajetória do algoritmo de Karmarkar, sob determinadas condições. 


\section{4) RELAÇÃO ENTRE O ALGORITMO DE KARMARKAR E O MÉTODO DA BARREIRA DE NEWTON PROJETADA}

Para problemas de programação linear na forma apresentada em (3.11) e para uma dada escolha do parâmetro de barreira e do tamanho do passo, o algoritmo de Karmarkar e o método da barreira de Newton projetada são idênticos, partindo do mesmo ponto inicial.

Para isto algumas condições deverão ser satisfeitas, conforme proposto por GILL et al (1986).

- Parâmetro de barreira :

$O$ valor de $\mu$ deverá ser escolhido tal que satisfaça a seguinte igualdade:

$\mu=x^{t} r_{B}$

onde:

$r_{B}=D c-D A^{t} \pi_{B} ;$
$D=\operatorname{diag}\left\{x_{1}, x_{2}, \cdots, x_{n}\right\}$.

Para encontrar o valor de $\pi_{\mathrm{B}}$, será necessário resolver o seguinte problema:

$$
\min _{\pi_{i}}\left\|\mathrm{Dc}-\mathrm{DA}^{\mathrm{t}} \pi_{\mathrm{B}}\right\|
$$

Conhecendo o valor de $\pi_{\mathrm{B}}$ através de (3.25), pode-se calcular o vetor $\mathrm{r}_{\mathrm{B}}$ utilizando (3.24). 
- Tamanho do passo $\alpha$ :

Para se obter o tamanho do passo calculado a cada iteração para a atualização das variáveis, será primeiramente necessário definir as variáveis $\alpha_{\mathrm{K}} \mathrm{e}$ w:

$\alpha_{\mathrm{K}}=\frac{\rho \mathrm{r}}{\left\|\mathrm{r}_{\mathrm{K}}\right\|} \mathrm{n}$

onde:

$\mathrm{r}_{\mathrm{K}}=\mathrm{r}_{\mathrm{B}}-\phi \mathrm{e} ;$

$\phi=c^{t} x / n$;

r e $\rho$ definidos em (3.19) e (3.22) respectivamente.

$w=\frac{1}{1+\alpha_{K}(\phi-\mu)}$

Conhecendo as variáveis $\alpha_{\mathrm{K}}$ e w o tamanho do passo utilizado no método da barreira de Newton projetada deverá ser calculado através de:

$\alpha=\alpha_{\mathrm{K}} \mathrm{w} \mu$

Se o parâmetro de barreira e o tamanho do passo, no método da barreira de Newton projetada, forem calculados conforme (3.23) e (3.28) respectivamente, a trajetória seguida pelos dois métodos serão idênticas, como será mostrado no próximo item. 
$\mathrm{O}$ vetor $r_{\mathrm{K}}$ é idêntico ao vetor $c_{\mathrm{p}}$, utilizado no algoritmo de Karmarkar definido em (3.21). Porém o vetor $\mathrm{r}_{\mathrm{K}}$ é obtido de uma forma mais simplificada, evitando assim as muitas operações matriciais realizadas no algoritmo de Karmarkar.

\section{5) EXEMPLO}

O exemplo a seguir foi implementado em Linguagem $\mathrm{C}$, em ambas as técnicas de otimização já citadas. No método da barreira de Newton projetada, o parâmetro de barreira e o tamanho do passo, atualizados a cada iteração, foram calculados conforme (3.23) e (3.28) respectivamente. O valor da tolerância desejada $\varepsilon$ foi de 0.00001 em ambos os métodos.

Considere o seguinte problema de programação linear, no formato padrão de $(3.11)$.

$$
\begin{array}{lc}
\min & x_{2} \\
\text { s. a } & x_{1}+x_{2}-2 x_{3}=0 \\
& x_{1}+x_{2}+x_{3}=1 \\
& x_{1}, \quad x_{2}, \quad x_{3} \geq 0
\end{array}
$$

Na tabela 1 tem-se os resultados obtidos após cada iteração de ambos os métodos de otimização. 
TABELA 1 - Resultados dos métodos de Karmarkar e barreira de Newton projetada.

método de Karmarkar método da barreira de

Newton projetada

\begin{tabular}{|c|c|c|c|c|c|c|c|c|c|c|}
\hline iteração & $\mathrm{xl}$ & $x 2$ & $x 3$ & fo & iteracão & xl & $x 2$ & $\mathrm{x} 3$ & u & fo \\
\hline 1 & 0.3333 & 0.3333 & 0.3333 & 0.3333 & 1 & 0.3333 & 0.3333 & 0.3333 & 0.1111 & 0.3333 \\
\hline 2 & 0.3975 & 0.2692 & 0.3333 & 0.2692 & 2 & 0.3975 & 0.2692 & 0.3333 & 0.0716 & 0.2692 \\
\hline 3 & 0.4574 & 0.2093 & 0.3333 & 0.2093 & 3 & 0.4574 & 0.2093 & 0.3333 & 0.0419 & 0.2093 \\
\hline 4 & 0.5090 & 0.1576 & 0.3333 & 0.1576 & 4 & 0.5090 & 0.1576 & 0.3333 & 0.0227 & 0.1576 \\
\hline 5 & 0.5507 & 0.1160 & 0.3333 & 0.1160 & 5 & 05507 & 0.1160 & 0.3333 & 0.0118 & 0.1160 \\
\hline 6 & 0.5827 & 0.0840 & 0.3333 & 0.0840 & 6 & 0.5827 & 0.0840 & 0.3333 & 0.0059 & 0.0840 \\
\hline 7 & 0.6065 & 0.0602 & 0.3333 & 0.0602 & 7 & 0.6065 & 0.0602 & 0.3333 & 0.0030 & 0.0602 \\
\hline 8 & 0.6239 & 0.0428 & 0.3333 & 0.0428 & 8 & 0.6239 & 0.0428 & 0.3333 & 0.0015 & 0.0428 \\
\hline 9 & 0.6364 & 0.0303 & 0.3333 & 0.0303 & 9 & 0.6364 & 0.0303 & 0.3333 & 0.0007 & 0.0303 \\
\hline 10 & 0.6453 & 0.0213 & 0.3333 & 0.0213 & 10 & 0.6453 & 0.0213 & 0.3333 & 0.0004 & 0.0213 \\
\hline 11 & 0.6517 & 0.0150 & 0.3333 & 0.0150 & 11 & 0.6517 & 0.0150 & 0.3333 & 0.0002 & 0.0150 \\
\hline 12 & 0.6561 & 0.0105 & 0.3333 & 0.0105 & 12 & 0.6561 & 0.0105 & 0.3333 & 0.0001 & 0.0105 \\
\hline 13 & 0.6593 & 0.0074 & 0.3333 & 0.0074 & 13 & 0.6593 & 0.0074 & 0.3333 & 0.0000 & 0.0074 \\
\hline 14 & 0.6615 & 0.0052 & 0.3333 & 0.0052 & 14 & 0.6615 & 0.0052 & 0.3333 & 0.0000 & 0.0052 \\
\hline 15 & 0.6630 & 0.0036 & 0.3333 & 0.0036 & 15 & 0.6630 & 0.0036 & 0.3333 & 0.0000 & 0.0036 \\
\hline 16 & 0.6641 & 0.0025 & 0.3333 & 0.0025 & 16 & 0.6641 & 0.0025 & 0.3333 & 0.0000 & 0.0025 \\
\hline 17 & 0.6649 & 0.0018 & 0.3333 & 0.0018 & 17 & 0.6649 & 0.0018 & 0.3333 & 0.0000 & 0.0018 \\
\hline 18 & 0.6654 & 0.0012 & 0.3333 & 0.0012 & 18 & 0.6654 & 0.0012 & 0.3333 & 0.0000 & 0.0012 \\
\hline 19 & 0.6658 & 0.0009 & 0.3333 & 0.0009 & 19 & 0.6658 & 0.0009 & 0.3333 & 0.0000 & 0.0009 \\
\hline 20 & 0.6661 & 0.0006 & 0.3333 & 0.0006 & 20 & 0.6661 & 0.0006 & 0.3333 & 0.0000 & 0.0006 \\
\hline 21 & 0.6662 & 0.0004 & 0.3333 & 0.0004 & 21 & 0.6662 & 0.0004 & 0.3333 & 0.0000 & 0.0004 \\
\hline 22 & 0.6664 & 0.0003 & 0.3333 & 0.0003 & 22 & 0.6664 & 0.0003 & 0.3333 & 0.0000 & 0.0003 \\
\hline 23 & 0.6665 & 0.0002 & 0.3333 & 0.0002 & 23 & 0.6665 & 0.0002 & 0.3333 & 0.0000 & 0.0002 \\
\hline 24 & 0.6665 & 0.0001 & 0.3333 & 0.0001 & 24 & 0.6665 & 0.0001 & 0.3333 & 0.0000 & 0.0001 \\
\hline 25 & 0.6666 & 0.0001 & 0.3333 & 0.0001 & 25 & 0.6666 & 0.0001 & 0.3333 & 0.0000 & 0.0001 \\
\hline 26 & 0.6666 & 0.0001 & 0.3333 & 0.0001 & 26 & 0.6666 & 0.0001 & 0.3333 & 0.0000 & 0.0001 \\
\hline 27 & 0.6666 & 0.0001 & 0.3333 & 0.0001 & 27 & 0.6666 & 0.0001 & 0.3333 & 0.0000 & 0.0001 \\
\hline 28 & 0.6666 & 0.0000 & 0.3333 & 0.0000 & 28 & 0.6666 & 0.0000 & 0.3333 & 0.0000 & 0.0000 \\
\hline 29 & 0.6666 & 0.0000 & 0.3333 & 0.0000 & 29 & 0.6666 & 0.0000 & 0.3333 & 0.0000 & 0.0000 \\
\hline 30 & 0.6666 & 0.0000 & 0.3333 & 0.0000 & 30 & 0.6666 & 0.0000 & 0.3333 & 0.0000 & 0.0000 \\
\hline 31 & 0.6667 & 0.0000 & 0.3333 & 0.0000 & 31 & 0.6667 & 0.0000 & 0.3333 & 0.0000 & 0.0000 \\
\hline
\end{tabular}

Como pôde ser verificado, ambos os métodos seguiram a mesma trajetória, sob as condições apresentadas anteriormente. Concluímos então que para solução de problemas lineares de otimização na forma dada em (3.11), é mais vantajoso implementar o método da barreira de Newton projetada, o qual utiliza a função barreira logarítmica definida em (3.1), pois não tem a necessidade de realizar muitas operações matriciais, como vimos que ocorre no algoritmo de Karmarkar. 


\section{O MÉTODO PRIMAL DUAL BARREIRA LOGARÍTMICA APLICADO AO PROBleMA DE FLUXO DE CARGA ÓTIMO}

\section{1) INTRODUÇÃO}

A análise do fluxo de carga convencional abrange o cálculo dos fluxos de potência, das tensões, dos ângulos e dos taps dos transformadores em um sistema de transmissão, para uma carga especificada e um programa de geração estabelecido.

A modelagem do sistema elétrico de potência é estática, isto é, a rede é representada por um conjunto de equações e inequações algébricas, não-lineares e lineares. As equações não-lineares do fluxo de carga são obtidas impondo-se o princípio da conservação das potências ativas e reativas em cada barra do sistema, as inequações lineares são dadas pelas restrições das magnitudes das tensões e pelos taps dos transformadores, enquanto que as desigualdades não-lineares correspondem aos limites das injeções de potência reativa nas barras de geração.

A solução das equações e inequações que constituem o modelo estático da rede pode ser realizada por vários métodos. Entre eles podemos destacar o método de Newton e suas variações, sendo o método desacoplado rápido uma delas. Estas técnicas de solução ajustam alguns parâmetros de controle com base em algum critério local, como por exemplo, manter a tensão em uma dada barra dentro de seus limites através do ajuste do tap do transformador, porém quando o sistema possui 
dimensão muito elevada, ajustar muitas variáveis simultaneamente pode levar muito tempo, além de ser um trabalho exaustivo, e é nesta área que o fluxo de carga ótimo toma o seu lugar de destaque, pois visa ajustar as variáveis de controle de acordo com um critério global, ao invés de local, fornecendo assim um ponto de operação que otimiza um determinado objetivo satisfazendo as restrições do problema.

\section{2) O PROBLEMA DE FLUXO DE CARGA}

Como apresentado por MONTICELLI (1983), o fluxo de carga pode ser formulado por equações e inequações lineares e não-lineares as quais representam as restrições de operação do sistema elétrico de potência. Para a formulação do problema quatro variáveis estão associadas a cada barra do sistema. São elas:

- magnitude da tensão na barra $\mathrm{k}, \mathrm{V}_{\mathrm{k}}$

- ângulo da tensão na barra $\mathrm{k}, \theta_{\mathrm{k}}$

- geração líquida de potência ativa na barra $\mathrm{k}, \mathrm{P}_{\mathrm{k}}$

- geração líquida de potência reativa na barra k, $Q_{k}$

Entre estas variáveis, duas são incógnitas (calculadas) e duas são dados do problema, e esta divisão classifica as barras do sistema em três tipos. São eles:

- barra de referência ou $\theta \mathrm{V}$ :

- dados $V_{k}$ e $\theta_{k}$

- calculados $\mathrm{P}_{\mathrm{k}}$ e $\mathrm{Q}_{\mathrm{k}}$

-barra PQ:

- dados $\mathrm{P}_{\mathrm{k}}$ e $\mathrm{Q}_{\mathrm{k}}$

- calculados $\mathrm{V}_{\mathrm{k}}$ e $\theta_{\mathrm{k}}$ 
-barra PV:

- dados $P_{k}$ e $V_{k}$

- calculados $\mathrm{Q}_{\mathrm{k}}$ e $\theta_{\mathrm{k}}$

A barra de $\theta \mathrm{V}$ fornece a referência angular do sistema e é utilizada para fechar o balanço de potência ativa, enquanto as barras PQ e PV são barras de carga e de geração respectivamente. Cada barra do sistema corresponde a duas equações nãolineares que são obtidas impondo-se a Primeira Lei de Kirchhoff, isto é, as injeções de potências ativas e reativas em uma barra é igual a soma dos fluxos correspondentes que deixam a barra através das linhas de transmissão. Estas equações podem ser matematicamente expressas por:

$$
\begin{aligned}
& \Delta P_{k}=P_{k}^{G}-P_{k}^{C}-V_{k} \sum_{m \in K} V_{m}\left(G_{k m} \cos \theta_{k m}+B_{k m} \operatorname{sen} \theta_{k m}\right)=0 \\
& \Delta Q_{k}=Q_{k}^{G}-Q_{k}^{C}-V_{k} \sum_{m \in K} V_{m}\left(G_{k m} \operatorname{sen} \theta_{k m}-B_{k m} \cos \theta_{k m}\right)=0
\end{aligned}
$$

onde:

$\mathrm{P}_{\mathrm{k}}^{\mathrm{G}}$ é potência ativa gerada na barra $\mathrm{k}$;

$\mathrm{P}_{\mathrm{k}}^{\mathrm{C}}$ potência ativa consumida na barra $\mathrm{k}$;

$\mathrm{Q}_{\mathrm{k}}^{\mathrm{G}}$ potência reativa gerada na barra $\mathrm{k}$;

$\mathrm{Q}_{\mathrm{k}}^{\mathrm{C}}$ potência reativa consumida na barra $\mathrm{k}$;

$\mathrm{V}_{\mathrm{k}}$ é tensão na barra $\mathrm{k}$;

$\mathrm{V}_{\mathrm{m}}$ é tensão na barra $\mathrm{m}$;

$\theta_{\mathrm{km}}=\theta_{\mathrm{k}}-\theta_{\mathrm{m}}$, ângulo da tensão na barra $\mathrm{k}$ e m, respectivamente;

$\mathrm{G}_{\mathrm{km}}$ e $\mathrm{B}_{\mathrm{km}}$, elementos da matriz admitância nodal;

$\mathrm{K}$ é o conjunto de todas as barras vizinhas a barra $\mathrm{k}$, incluindo ela mesma.

As restrições de operação do problema de fluxo de carga são dadas por:

- limite das tensões:

$$
\mathrm{V}_{\mathrm{k}}^{\min } \leq \mathrm{V}_{\mathrm{k}} \leq \mathrm{V}_{\mathrm{k}}^{\max }
$$


- limite dos taps dos transformadores:

$$
\operatorname{tap}_{i}^{\min } \leq \operatorname{tap} \leq \operatorname{tap}_{i}^{\max }
$$

- limite de injeção de reativos nas barras PV:

$$
\mathrm{Q}_{\mathrm{k}}^{\min } \leq \mathrm{Q}_{\mathrm{k}} \leq \mathrm{Q}_{\mathrm{k}}^{\max }
$$

onde:

$\mathrm{Q}_{\mathrm{k}}=\mathrm{V}_{\mathrm{k}} \sum_{\mathrm{m} \in \mathrm{K}} \mathrm{V}_{\mathrm{m}}\left(\mathrm{G}_{\mathrm{km}} \operatorname{sen} \theta_{\mathrm{km}}-\mathrm{B}_{\mathrm{km}} \cos \theta_{\mathrm{km}}\right)$

Utilliza-se o método de Newton para encontrar a solução de (4.1) e após algumas iterações o ponto de operação do sistema $\left(V_{k}, \theta_{k}\right)$ é obtido. O método de Newton resolve o seguinte sistema linearizado a cada iteração:

$$
\left[\begin{array}{l}
\Delta \mathrm{P} \\
\Delta \mathrm{Q}
\end{array}\right]=\left[\begin{array}{ll}
\mathrm{H} & \mathrm{N} \\
\mathrm{M} & \mathrm{L}
\end{array}\right]\left[\begin{array}{c}
\Delta \theta \\
\Delta \mathrm{V}
\end{array}\right]
$$

onde:

H, N, M, L são submatrizes, as quais formam a matriz Jacobiana, que corresponde a primeira derivada das equações dadas em (4.1) em relação a $V_{k}$ e a $\theta_{k}$. As componentes das submatrizes são dadas por:

$$
\begin{aligned}
& \mathrm{H}\left\{\begin{array}{l}
\mathrm{H}_{\mathrm{km}}=\partial \Delta \mathrm{P}_{\mathrm{k}} / \partial \theta_{\mathrm{m}}=\mathrm{V}_{\mathrm{k}} \mathrm{V}_{\mathrm{m}}\left(\mathrm{G}_{\mathrm{km}} \operatorname{sen} \theta_{\mathrm{km}}-\mathrm{B}_{\mathrm{km}} \cos \theta_{\mathrm{km}}\right) \\
\mathrm{H}_{\mathrm{kk}}=\partial \Delta \mathrm{P}_{\mathrm{k}} / \partial \theta_{\mathrm{k}}=-\mathrm{V}_{\mathrm{k}}^{2} \mathrm{~B}_{\mathrm{kk}}-\mathrm{V}_{\mathrm{k}} \sum_{\mathrm{m} \in \mathrm{K}} \mathrm{V}_{\mathrm{m}}\left(\mathrm{G}_{\mathrm{km}} \operatorname{sen} \theta_{\mathrm{km}}-\mathrm{B}_{\mathrm{km}} \cos \theta_{\mathrm{km}}\right)
\end{array}\right. \\
& \mathrm{N}\left\{\begin{array}{l}
\mathrm{N}_{\mathrm{km}}=\partial \Delta \mathrm{P}_{\mathrm{k}} / \partial \mathrm{V}_{\mathrm{m}}=\mathrm{V}_{\mathrm{k}}\left(\mathrm{G}_{\mathrm{km}} \cos \theta_{\mathrm{km}}+\mathrm{B}_{\mathrm{km}} \operatorname{sen} \theta_{\mathrm{km}}\right) \\
\mathrm{N}_{\mathrm{kk}}=\partial \Delta \mathrm{P}_{\mathrm{k}} / \partial \mathrm{V}_{\mathrm{k}}=\mathrm{V}_{\mathrm{k}} \mathrm{G}_{\mathrm{kk}}+\sum_{\mathrm{m} \in \mathrm{K}} \mathrm{V}_{\mathrm{m}}\left(\mathrm{G}_{\mathrm{km}} \cos \theta_{\mathrm{km}}+\mathrm{B}_{\mathrm{km}} \operatorname{sen} \theta_{\mathrm{km}}\right)
\end{array}\right.
\end{aligned}
$$




$$
\begin{gathered}
M\left\{\begin{array}{l}
M_{k m}=\partial \Delta Q_{k} / \partial \theta_{m}=-V_{k} V_{m}\left(G_{k m} \cos \theta_{k m}+B_{k m} \operatorname{sen} \theta_{k m}\right) \\
M_{k k}=\partial \Delta Q_{k} / \partial \theta_{k}=-V_{k}^{2} G_{k k}+V_{k} \sum_{m \in K} V_{m}\left(G_{k m} \cos \theta_{k m}+B_{k m} \operatorname{sen} \theta_{k m}\right)
\end{array}\right. \\
L\left\{\begin{array}{l}
L_{k m}=\partial \Delta Q_{k} / \partial V_{m}=V_{k}\left(G_{k m} \operatorname{sen} \theta_{k m}-B_{k m} \cos \theta_{k m}\right) \\
L_{k k}=\partial \Delta Q_{k} / \partial V_{k}=-V_{k} B_{k k}+\sum_{m \in K} V_{m}\left(G_{k m} \operatorname{sen} \theta_{k m}-B_{k m} \cos \theta_{k m}\right)
\end{array}\right.
\end{gathered}
$$

Durante o processo de solução do sistema de equações dadas em (4.5), testes são realizados para verificar se as restrições de operação estão sendo violadas ou não. Como exemplo de restrição de operação, podemos citar os limites na geração de potência reativa nas barras PV. Se um desses limites, inferior ou superior, for violado, o valor de $\mathrm{Q}_{k}$ será fixado no valor limite correspondente, e a barra PV será transformada em uma barra $\mathrm{PQ}$, sendo que neste caso o valor da tensão não poderá ser fixado. Em iterações seguintes esta barra poderá voltar a ser do tipo PV. Contudo poderão acontecer situações em que esta técnica não será suficiente para fornecer um ponto adequado de operação para sistema elétrico de potência. Nestas condições, o operador do sistema terá que ajustar as variáveis de controle com base em sua própria experiência e rodar novamente o programa de fluxo de carga para obter um novo ponto de operação. Neste contexto o FCO surge como uma ferramenta muito atrativa, pois o ponto que fornece, automaticamente já satisfaz as restrições de desigualdade, evitando desta forma uma provável atuação do operador.

\section{3) O PROBLEMA DE FLUXO DE CARGA ÓTIMO}

Um sistema elétrico de potência tem por objetivo fornecer energia dentro de padrões de qualidade e confiabilidade para seus consumidores. Para isto ser possível, é necessário que todas as variáveis do sistema estejam dentro de seus limites previamente estabelecidos. Modificações na configuração da rede, alterações na demanda do sistema, podem fazer com que as tensões atinjam valores muito elevados ou reduzidos, violando desta forma os seus limites. Como mencionado anteriormente, 
um operador experiente, utilizando o fluxo de carga convencional, pode corrigir esta indesejável operação ajustando as variáveis de controle para situações localizadas, porém quando o sistema é de grande porte, um grande número de variáveis precisam ser ajustadas para satisfazer as condições de operação do sistema, e o uso do fluxo de carga convencional torna-se um exaustivo processo de tentativas e erros. Nestas condições, o fluxo de carga ótimo é o instrumento indicado para determinar o melhor ponto de operação do sistema de potência, ajustando simultaneamente e de maneira ótima, todas as variáveis de controle. Este ponto ótimo otimiza uma dada função objetivo e satisfaz as restrições de operação.

O problema de fluxo de carga ótimo pode ser formulado da seguinte maneira:

$\min f(x)$

$$
\begin{array}{ll}
\text { s. a } & \mathrm{g}(\mathrm{x})=0 \\
& \mathrm{~h}_{1} \leq \mathrm{h}(\mathrm{x}) \leq \mathrm{h}_{\mathrm{u}} \\
& \mathrm{l} \leq \mathrm{x} \leq \mathrm{u}
\end{array}
$$

onde:

$\mathrm{f}(\mathrm{x})$ é a função objetivo a ser otimizada;

$\mathrm{g}(\mathrm{x})$ é o vetor que corresponde às equações (4.1) do fluxo de carga convencional;

$\mathrm{h}(\mathrm{x})$ é o vetor que corresponde à inequação (4.4) do fluxo de carga convencional; $\mathrm{x}$ corresponde às variáveis de estado do sistema;

$h_{1}$ e $h_{u}$ são os limites inferiores e superiores de $h(x)$, respectivamente; l e u são os limites inferiores e superiores de x, respectivamente.

Como foi visto no capítulo 2 , existem muitas técnicas de otimização para solução do problema (4.10). A técnica de solução utilizando pontos interiores tem sido vista como uma alternativa eficiente para solução de problemas de otimização de sistemas de potência. Entre os métodos de otimização que utilizam pontos interiores, o método primal-dual barreira-logarítmica, tem sido amplamente aplicado para 
solução de (4.10). Podemos destacar nesta área os trabalhos de GRANVILLE (1994), WU et al. (1994), QUINTANA et al. (1995) e IRISSARI et al. (1997). A seguir será apresentado o método primal-dual barreira-logarítmica.

\section{4) MÉTODO PRIMAL-DUAL BARREIRA-LOGARÍTMICA}

Neste item será apresentado o método primal-dual barreira-logarítmica conforme GRANVILLE (1994). Para a aplicação deste método no problema apresentado em (4.10), algumas modificações deverão ser feitas. Desta forma, o problema (4.10) passa a ser reescrito da seguinte maneira:

$$
\begin{array}{ll}
\min & f(x) \\
\text { s. a } & g(x)=0 \\
& h(x)+s_{1}=h_{u} \\
& h(x)-s_{2}=h_{1} \\
& x+s_{3}=u \\
& x-s_{4}=1 \\
& s_{1}, s_{2}, s_{3}, s_{4}>0
\end{array}
$$

Observe que se desmembrou as restrições canalizadas em duas inequações e se acrescentou variáveis de folga $\left(s_{1}, s_{2}, s_{3}, s_{4}\right)$ estritamente positivas, para que estas restrições se transformassem em igualdades.

Estas variáveis de folga serão acrescentadas na função objetivo através da função barreira-logarítmica definida em (3.1), desta forma (4.11) passa a ser reescrito da seguinte maneira: 
$\min f(x)-\mu \sum_{i=1}^{n c r} \ln \left(s_{1 i}\right)-\mu \sum_{i=1}^{n c r} \ln \left(s_{2 i}\right)-\mu \sum_{i=1}^{n b} \ln \left(s_{3 i}\right)-\mu \sum_{i=1}^{n b} \ln \left(s_{4 i}\right)$

$$
\begin{array}{ll}
\text { s. a } & g(x)=0 \\
& h(x)+s_{1}=h_{u} \\
& h(x)-s_{2}=h_{1} \\
& x+s_{3}=u \\
& x-s_{4}=1
\end{array}
$$

onde:

nb é o número de barras do sistema;

ncr é o número de barras de controle de reativo;

$\mu(\mu>0)$ é o parâmetro de barreira, o qual tende a zero durante o processo de otimização, isto é, $\mu_{0}>\mu_{1}>\ldots>\mu_{\infty}=0$.

Com o objetivo de tornar o problema restrito (4.12) em um problema modificado irrestrito, a função Lagrangeana é construída e é dada por:

$$
\begin{aligned}
\mathrm{L}= & \mathrm{f}(\mathrm{x})-\mu \sum_{\mathrm{i}=1}^{\mathrm{ncr}} \ln \left(\mathrm{s}_{1 \mathrm{i}}\right)-\mu \sum_{\mathrm{i}=1}^{\mathrm{ncr}} \ln \left(\mathrm{s}_{2 \mathrm{i}}\right)-\mu \sum_{\mathrm{i}=1}^{\mathrm{nb}} \ln \left(\mathrm{s}_{3 \mathrm{i}}\right)-\mu \sum_{\mathrm{i}=1}^{\mathrm{nb}} \ln \left(\mathrm{s}_{4 \mathrm{i}}\right) \\
& -\lambda^{\mathrm{t}} \mathrm{g}(\mathrm{x})-\pi_{1}\left(\mathrm{~h}(\mathrm{x})+\mathrm{s}_{1}-\mathrm{h}_{\mathrm{u}}\right)-\pi_{2}\left(\mathrm{~h}(\mathrm{x})-\mathrm{s}_{2}-\mathrm{h}_{\mathrm{l}}\right) \\
& -\pi_{3}\left(\mathrm{x}+\mathrm{s}_{3}-\mathrm{u}\right)-\pi_{4}\left(\mathrm{x}-\mathrm{s}_{4}-\mathrm{l}\right)
\end{aligned}
$$

onde:

$\lambda, \pi_{1}, \pi_{2}, \pi_{3}$ e $\pi_{4}$ são os vetores dos multiplicadores de Lagrange.

Observe que a função Lagrangeana é construída acrescentando-se todas as restrições de igualdade à função objetivo, através de multiplicadores de Lagrange. 
Aplicando as condições de otimalidade em (4.13), obtém-se o seguinte sistema de equações:

$$
\begin{aligned}
& \nabla_{\mathrm{x}} \mathrm{L}=\nabla_{\mathrm{x}} \mathrm{f}(\mathrm{x})-\nabla_{\mathrm{x}} \mathrm{g}(\mathrm{x})^{\mathrm{t}} \lambda-\nabla_{\mathrm{x}} \mathrm{h}(\mathrm{x})^{\mathrm{t}} \pi_{1}-\nabla_{\mathrm{x}} \mathrm{h}(\mathrm{x})^{\mathrm{t}} \pi_{2}-\pi_{3}-\pi_{4}=0 \\
& \nabla_{\lambda} \mathrm{L}=-\mathrm{g}(\mathrm{x})=0 \\
& \nabla_{\pi_{1}} \mathrm{~L}=-\left(\mathrm{h}(\mathrm{x})+\mathrm{s}_{1}-\mathrm{h}_{\mathrm{u}}\right)=0 \\
& \nabla_{\pi_{2}} \mathrm{~L}=-\left(\mathrm{h}(\mathrm{x})-\mathrm{s}_{2}-\mathrm{h}_{1}\right)=0 \\
& \nabla_{\pi_{3}} \mathrm{~L}=-\left(\mathrm{x}+\mathrm{s}_{3}-\mathrm{u}\right)=0 \\
& \nabla_{\pi_{4}} \mathrm{~L}=-\left(\mathrm{x}-\mathrm{S}_{4}-\mathrm{l}\right)=0 \\
& \nabla_{\mathrm{S}_{1}} \mathrm{~L}=-\mu \mathrm{S}_{1}^{-1} \mathrm{e}-\pi_{1}=0 \\
& \nabla_{\mathrm{S}_{2}} \mathrm{~L}=-\mu \mathrm{S}_{2}^{-1} \mathrm{e}+\pi_{2}=0 \\
& \nabla_{\mathrm{S}_{3}} \mathrm{~L}=-\mu \mathrm{S}_{3}^{-1} \mathrm{e}-\pi_{3}=0 \\
& \nabla_{\mathrm{S}_{4}} \mathrm{~L}=-\mu \mathrm{S}_{4}^{-1} \mathrm{e}+\pi_{4}=0
\end{aligned}
$$

onde:

$e^{t}=(1,1, \ldots, 1)$;

$\mathrm{S}_{1}, \mathrm{~S}_{2}, \mathrm{~S}_{3}, \mathrm{~S}_{4}$ são matrizes diagonais, cujos elementos são $\mathrm{s}_{1}, \mathrm{~s}_{2}, \mathrm{~s}_{3}, \mathrm{~s}_{4}$, respectivamente.

O método de Newton é utilizado para solucionar o sistema de equações (4.14) a (4.23). Este método utiliza a expansão em série de Taylor até primeira ordem das equações do sistema, e desta forma gera as direções de busca $\left(\Delta \mathrm{x}, \Delta \lambda, \Delta \pi_{1}, \Delta \pi_{2}, \Delta \pi_{3}, \Delta \pi_{4}, \Delta \mathrm{s}_{1}, \Delta \mathrm{s}_{2}, \Delta \mathrm{s}_{3}, \Delta \mathrm{s}_{4}\right)$, que serão utilizadas para a atualização das variáveis do sistema. Desta forma, as equações de Newton para se obter as direções são as seguintes: 


$$
\begin{aligned}
& \mathrm{W}\left(\mathrm{x}, \lambda, \pi_{1}, \pi_{2}\right) \Delta \mathrm{x}-\nabla_{\mathrm{x}} \mathrm{g}(\mathrm{x})^{\mathrm{t}} \Delta \lambda_{\mathrm{u}}-\nabla_{\mathrm{x}} \mathrm{h}(\mathrm{x})^{\mathrm{t}} \Delta \pi_{1}-\nabla_{\mathrm{x}} \mathrm{h}(\mathrm{x})^{\mathrm{t}} \Delta \pi_{2}-\Delta \pi_{3}-\Delta \pi_{4}=-\nabla_{\mathrm{x}} \mathrm{L} \\
& -\left(\nabla_{\mathrm{x}} \mathrm{g}(\mathrm{x})\right) \Delta \mathrm{x}=-\nabla_{\lambda} \mathrm{L} \\
& -\left(\nabla_{\mathrm{x}} \mathrm{h}(\mathrm{x}) \Delta \mathrm{x}+\Delta \mathrm{s}_{1}\right)=-\nabla_{\pi_{1}} \mathrm{~L} \\
& -\left(\nabla_{\mathrm{x}} \mathrm{h}(\mathrm{x}) \Delta \mathrm{x}-\Delta \mathrm{s}_{2}\right)=-\nabla_{\pi_{2}} \mathrm{~L} \\
& -\left(\Delta \mathrm{x}+\Delta \mathrm{s}_{3}\right)=-\nabla_{\pi_{3}} \mathrm{~L} \\
& -\left(\Delta \mathrm{x}-\Delta \mathrm{s}_{4}\right)=-\nabla_{\pi_{4}} \mathrm{~L} \\
& \mu \mathrm{S}_{1}^{-2} \Delta \mathrm{s}_{1}-\Delta \pi_{1}=-\nabla_{\mathrm{s}_{1}} \mathrm{~L} \\
& \mu \mathrm{S}_{2}^{-2} \Delta \mathrm{s}_{2}+\Delta \pi_{2}=-\nabla_{\mathrm{s}_{2}} \mathrm{~L} \\
& \mu \mathrm{S}_{3}^{-2} \Delta \mathrm{s}_{3}-\Delta \pi_{3}=-\nabla_{\mathrm{s}_{3}} \mathrm{~L} \\
& \mu \mathrm{S}_{4}^{-2} \Delta \mathrm{s}_{4}+\Delta \pi_{4}=-\nabla_{\mathrm{S}_{4}} \mathrm{~L}
\end{aligned}
$$

Reescrevendo (4.24) na forma matricial, tem-se o seguinte:

$$
\left[\begin{array}{cccccccccc}
\mathrm{W}\left(\mathrm{x}, \lambda, \pi_{1}, \pi_{2}\right) & -\mathrm{J}(\mathrm{x})^{\mathrm{t}} & -\nabla_{\mathrm{x}} \mathrm{h}(\mathrm{x})^{\mathrm{t}} & -\nabla_{\mathrm{x}} \mathrm{h}(\mathrm{x})^{\mathrm{t}} & -\mathrm{I} & -\mathrm{I} & 0 & 0 & 0 & 0 \\
-\mathrm{J}(\mathrm{x}) & 0 & 0 & 0 & 0 & 0 & 0 & 0 & 0 & 0 \\
-\nabla_{\mathrm{x}} \mathrm{h}(\mathrm{x}) & 0 & 0 & 0 & 0 & 0 & -\mathrm{I} & 0 & 0 & 0 \\
-\nabla_{\mathrm{x}} \mathrm{h}(\mathrm{x}) & 0 & 0 & 0 & 0 & 0 & 0 & \mathrm{I} & 0 & 0 \\
-\mathrm{I} & 0 & 0 & 0 & 0 & 0 & 0 & 0 & -\mathrm{I} & 0 \\
-\mathrm{I} & 0 & 0 & 0 & 0 & 0 & 0 & 0 & 0 & \mathrm{I} \\
0 & 0 & -\mathrm{I} & 0 & 0 & 0 & \mu \mathrm{S}_{1}^{-2} & 0 & 0 & 0 \\
0 & 0 & 0 & \mathrm{I} & 0 & 0 & 0 & \mu \mathrm{S}_{2}^{-2} & 0 & 0 \\
0 & 0 & 0 & 0 & -\mathrm{I} & 0 & 0 & 0 & \mu \mathrm{S}_{3}^{-2} & 0 \\
0 & 0 & 0 & 0 & 0 & \mathrm{I} & 0 & 0 & 0 & \mu \mathrm{S}_{4}^{-2}
\end{array}\right]\left[\begin{array}{c}
\Delta \mathrm{x} \\
\Delta \lambda_{\mathrm{L}} \\
\Delta \pi_{1} \\
\Delta \pi_{2} \\
\Delta \pi_{3} \\
\Delta \pi_{4} \\
\Delta \mathrm{s}_{1} \\
\Delta \mathrm{s}_{2} \\
\Delta \mathrm{s}_{3} \\
\Delta \mathrm{s}_{4}
\end{array}\right]=-\left[\begin{array}{c}
\nabla_{\mathrm{x}} \mathrm{L} \\
\nabla_{\lambda_{2}} \mathrm{~L} \\
\nabla_{\pi_{1}} \mathrm{~L} \\
\nabla_{\pi_{3}} \mathrm{~L} \\
\nabla_{\pi_{3}} \mathrm{~L} \\
\nabla_{\pi_{4}} \mathrm{~L} \\
\nabla_{\mathrm{s}_{1}} \mathrm{~L} \\
\nabla_{\mathrm{s}_{2}} \mathrm{~L} \\
\nabla_{\mathrm{s}_{1}} \mathrm{~L} \\
\nabla_{\mathrm{s}_{1}} \mathrm{~L}
\end{array}\right]
$$

onde:

$\mathrm{W}\left(\mathrm{x}, \lambda, \pi_{1}, \pi_{2}\right)=\nabla^{2} \mathrm{f}(\mathrm{x})-\sum_{\mathrm{i}=1}^{2 \mathrm{xnb}} \lambda_{\mathrm{i}} \nabla^{2} \mathrm{~g}_{\mathrm{i}}(\mathrm{x})-\sum_{\mathrm{i}=1}^{\mathrm{ner}} \pi_{1 \mathrm{i}} \nabla^{2} \mathrm{~h}(\mathrm{x})-\sum_{\mathrm{i}=1}^{\mathrm{ncr}} \pi_{2 \mathrm{i}} \nabla^{2} \mathrm{~h}(\mathrm{x}) ;$

$\mathrm{J}(\mathrm{x})=\nabla_{\mathrm{x}} \mathrm{g}(\mathrm{x})$, é a matriz Jacobiana do fluxo de carga convencional, mostrada em (4.5);

I é a matriz identidade. 
A matriz Lagrangeana do sistema (4.25) é esparsa, ou seja, possui muitos elementos nulos, e esta característica deve ser explorada no processo de solução do sistema.

A dimensão da matriz Lagrangeana, construída devido a aplicação do método primal-dual barreira-logarítmica para solução do problema (4.10), é muito maior do que a dimensão da matriz utilizada nos métodos do Gradiente Reduzido, Penalidade, Newton entre outros. Nestes métodos o sistema a ser resolvido é apenas uma parte do sistema dado em (4.25), isto é:

$$
\left[\begin{array}{cc}
\mathrm{W}(\mathrm{x}, \lambda) & -\mathrm{J}(\mathrm{x})^{\mathrm{t}} \\
-\mathrm{J}(\mathrm{x}) & 0
\end{array}\right]\left[\begin{array}{c}
\Delta \mathrm{x} \\
\Delta \lambda
\end{array}\right]=-\left[\begin{array}{c}
\nabla_{\mathrm{x}} \mathrm{L} \\
\nabla_{\lambda} \mathrm{L}
\end{array}\right]
$$

Observe que a matriz Lagrangeana em (4.25), cuja dimensão total é dada por:

$$
\begin{aligned}
\text { dimensão }= & 8\left(\mathrm{n}^{\circ} \text { de barras do sistema }\right)+ \\
& 3\left(\mathrm{n}^{\circ} \text { de barras de controle de reativo }\right)+ \\
& 5\left(\mathrm{n}^{\circ} \text { de transformadores }\right)- \\
& 3\left(\mathrm{n}^{\circ} \text { de barras } \theta \text { V }\right)
\end{aligned}
$$

possui muitos elementos unitários e as submatrizes referentes as variáveis de folga são de fácil implementação. Desta forma o esforço computacional necessário para a obtenção de (4.25) não é muito elevado, quando comparado com os métodos que utilizam o sistema (4.26).

\subsection{1) TAMANHO DO PASSO}

Conhecendo as direções de busca, a próxima etapa é calcular os passos $\alpha_{\mathrm{p}} \mathrm{e}$ $\alpha_{d}$, os quais serão usados na atualização das variáveis primais e duais respectivamente. Estes passos são calculados de tal forma que cada componente das 
variáveis de folga primais $\left(\mathrm{s}_{\mathrm{i}}, \mathrm{i}=1,2,3,4\right)$ permaneçam estritamente positivas, e que os elementos do vetor $\left(\pi_{i}, i=1,2,3,4\right)$ permaneçam com os seus respectivos sinais, isto é $\pi_{1}<0, \pi_{2}>0, \pi_{3}<0, \pi_{4}>0$, conforme (4.20), (4.21), (4.22), (4.23) respectivamente.

Isto é feito encontrando o menor elemento entre o mínimo tamanho de passo de todos os componentes dos vetores, este menor elemento é então multiplicado por um fator menor que 1 (para garantir que o próximo ponto irá satisfazer as condições de positividade) e comparado com 1, o menor entre eles é então escolhido, como propõe GRANVILLE (1994), QUINTANA et al. (1995) e IRISARRI et al. (1997). Isto se traduz por:

$$
\begin{aligned}
& \alpha_{\mathrm{p}}=\min \left\{\sigma\left(\min _{\Delta \mathrm{s}_{1}<0} \frac{\mathrm{s}_{1}}{\left|\Delta \mathrm{s}_{1}\right|}, \min _{\Delta \mathrm{s}_{2}<0} \frac{\mathrm{s}_{2}}{\left|\Delta \mathrm{s}_{2}\right|}, \min _{\Delta \mathrm{s}_{\mathrm{s}}<0} \frac{\mathrm{s}_{3}}{\left|\Delta \mathrm{s}_{3}\right|}, \min _{\Delta \mathrm{s}_{\iota}<0} \frac{\mathrm{s}_{4}}{\left|\Delta \mathrm{s}_{4}\right|}\right), 1.0\right\} \\
& \alpha_{\mathrm{d}}=\min \left\{\sigma\left(\min _{\Delta \pi_{\mathrm{i}}>0} \frac{-\pi_{1}}{\left|\Delta \pi_{1}\right|}, \min _{\Delta \pi_{2}<0} \frac{\pi_{2}}{\left|\Delta \pi_{2}\right|}, \min _{\Delta \pi_{3}>0\left|\Delta \pi_{3}\right|} \frac{-\pi_{3}}{\Delta \pi_{4}<0\left|\Delta \pi_{4}\right|}, 1.0\right\}\right.
\end{aligned}
$$

onde:

$\sigma=0.9995$ é um valor determinado empiricamente, conforme GRANVILLE (1994), QUINTANA et al. (1995) e IRISSARI et al. (1997).

Conhecendo as direções através da solução de (4.25) e os passos primais e duais através de (4.28) e (4.29) respectivamente, todas as variáveis do problema podem ser atualizadas por:

$$
\begin{array}{ll}
\mathrm{x}=\mathrm{x}+\alpha_{\mathrm{p}} \Delta \mathrm{x} & \lambda=\lambda+\alpha_{\mathrm{d}} \Delta \lambda \\
\mathrm{s}_{1}=\mathrm{s}_{1}+\alpha_{\mathrm{p}} \Delta \mathrm{s}_{1} & \pi_{1}=\pi_{1}+\alpha_{\mathrm{d}} \Delta \pi_{1} \\
\mathrm{~s}_{2}=\mathrm{s}_{2}+\alpha_{\mathrm{p}} \Delta \mathrm{s}_{2} & \pi_{2}=\pi_{2}+\alpha_{\mathrm{d}} \Delta \pi_{2} \\
\mathrm{~s}_{3}=\mathrm{s}_{3}+\alpha_{\mathrm{p}} \Delta \mathrm{s}_{3} & \pi_{3}=\pi_{3}+\alpha_{\mathrm{d}} \Delta \pi_{3} \\
\mathrm{~s}_{4}=\mathrm{s}_{4}+\alpha_{\mathrm{p}} \Delta \mathrm{s}_{4} & \pi_{4}=\pi_{4}+\alpha_{\mathrm{d}} \Delta \pi_{4}
\end{array}
$$


Após as variáveis terem sido atualizadas, deve-se atualizar o parâmetro de barreira. Este é o ponto crítico do algoritmo primal-dual barreira-logarítmica.

\subsection{2) O PARÂMETRO DE BARREIRA}

Uma etapa muito importante no algoritmo primal-dual barreira-logarítmica é a escolha inicial do parâmetro de barreira $\mu$. GRANVILLE (1994) verificou que o valor do multiplicador $\mu$ para cada ponto é proporcional ao gap de dualidade. Este gap é a diferença entre o valor da função objetivo do problema primal e do problema dual. A cada iteração o valor de $\mu$ deverá ser calculado tal que o seu valor atual seja sempre inferior ao anterior, e desta forma GRANVILLE (1994) propõe um cálculo para a atualização de $\mu$ utilizando a equação (4.31), na qual o numerador corresponde ao gap de dualidade:

$$
\mu=\frac{-\left(s_{1} \pi_{1}+s_{3} \pi_{3}\right)+\left(s_{2} \pi_{2}+s_{4} \pi_{4}\right)}{2 n b \beta}
$$

onde:

$\beta>1$ é especificado pelo usuário.

Como visto em (4.31) o parâmetro $\mu$ é reduzido a cada iteração empiricamente, pois o valor de $\beta$ é determinado pelo usuário. Uma escolha não adequada deste parâmetro poderá também comprometer a convergência do método. Uma outra opção para atualização de $\mu$, é reduzi-lo de um valor $\beta$ especificado pelo usuário, a cada iteração, isto é:

$$
\mu^{(k+1)}=\frac{\mu^{k}}{\beta}
$$


$\mathrm{Na}$ implementação do algoritmo primal-dual barreira-logarítmica realizada neste trabalho, com o objetivo de resolver o problema de fluxo de carga ótimo, utilizou-se a equação (4.32) para a atualização do parâmetro de barreira, devido a sua eficiência e fácil implementação.

\subsection{3) INICIALIZAÇÃO DAS VARIÁVEIS}

Com respeito à inicialização das variáveis, a única exigência que deve ser observada é que as variáveis do sistema, ou seja, tensões, taps e injeções de reativo estejam dentro de seus limites preestabelecidos. As equações de balanço do sistema (4.1) e as inequações que foram transformadas em equações através das variáveis de folga, não precisam ser satisfeitas na inicialização do problema. As variáveis de folga $\left(s_{i}, i=1,2,3,4\right)$ podem ser inicializadas utilizando as equações (4.16), (4.17), (4.18), (4.19) respectivamente. Estas variáveis devem ser estritamente positivas, isto é esperado desde que as variáveis do sistema estejam dentro de seus limites. No entanto, se existir algum componente deste vetor que seja nulo ou negativo, o mesmo poderá assumir o valor 0.02, como propõe QUINTANA et al. (1995). Após ter inicializado os vetores referentes as variáveis de folga, os vetores dos multiplicadores de Lagrange $\left(\pi_{\mathrm{i}}, \mathrm{i}=1,2,3,4\right)$ podem ser inicializados utilizando (4.20), (4.21), (4.22), (4.23) respectivamente. $O$ valor inicial do parâmetro de barreira e o multiplicador $\beta$ para atualização de $\mu$ são determinados pelo usuário, como mencionado anteriormente.

\section{5)ALGORITMO SIMPLIFICADO}

O problema de fluxo de carga ótimo mostrado em (4.10), pode ser resolvido iterativamente através do método dos pontos interiores pelo seguinte algoritmo apresentado a seguir, conforme GRANVILLE (1994): 
Inicialização:

Colocar o problema original na forma mostrada em (4.12). Iniciar o vetor $\mathrm{x}=(\mathrm{V}, \theta, \mathrm{t})$, as variáveis de folga, os multiplicadores de Lagrange, o parâmetro de barreira $\mu$ e o parâmetro $\beta$ para a atualização de $\mu$. Determinar um valor para $\varepsilon, o$ qual será o critério de parada.

\section{Passo principal:}

1. Calcular o vetor gradiente.

2. Se todos os elementos do vetor gradiente forem menores que $\varepsilon$ então PARE.

3. Calcular a matriz Lagrangeana e resolver o sistema (4.25).

4. Calcular os passos por (4.28) e (4.29).

5. Atualizar as variáveis (4.30) e o parâmetro de barreira por (4.32).

6. Voltar ao passo 1 .

$\mathrm{O}$ valor inicial de $\mathrm{x}=(\mathrm{V}, \theta, \mathrm{t})$ deve pertencer à região factível do problema. $\mathrm{A}$ implementação computacional deste algoritmo e a técnica utilizada para solução do sistema (4.25), serão discutidas nas próximas seções.

\section{6) IMPLEMENTAÇÃO COMPUTACIONAL}

A implementação computacional do método primal-dual barreira-logarítmica apresentado neste trabalho, foi realizada em Linguagem $\mathrm{C}$, em uma estação de trabalho do tipo SUN SPARC STATION 20 do Laboratório de Sistemas Energia Elétrica - LSEE, do Departamento de Engenharia Elétrica da Escola de Engenharia de São Carlos - EESC/USP. 
A seguir tem-se as subrotinas implementadas, as quais formam o programa de fluxo de carga ótimo:

variáveis.c: declaração das variáveis que serão utilizadas no programa.

leitura.c: rotinas responsáveis pelas leituras dos dados de barras, linhas de transmissão e limites de tensão. Estas informações estão contidas em bancos de dados, os quais fornecem as informações iniciais sobre a rede elétrica (Apêndice 1).

inicia.c: rotinas responsáveis pela inicialização das variáveis, que são as variáveis de folga e multiplicadores de Lagrange.

topologia.c: rotina que fornece a topologia da rede, ou seja, descreve as ligações existentes entre as barras do sistema.

gradiente.c: rotina que fornece o vetor gradiente da função Lagrangeana.

lagrangeana.c: rotinas responsáveis pela construção da matriz Lagrangeana, a qual é construída em um formato vetorial.

ma28ad.f: rotina em Fortran responsável pela fatoração LU. Na chamada desta rotina, fornecemos como informação a matriz Lagrangeana no formato vetorial.

ma28cd.f: rotina em Fortran responsável pela solução do sistema de equações. Na chamada desta rotina, fornecemos como informação a matriz Lagrangeana e o vetor gradiente.

atualiza.c: rotinas responsáveis pela atualização das variáveis do problema de fluxo de carga ótimo e do parâmetro de barreira.

saída.c: rotina que fornece a saída dos resultados obtidos pelo programa. 
Como pôde ser observado, duas linguagens de programação foram utilizadas na implementação do problema de fluxo de carga ótimo, Fortran e Linguagem $C$, razão pela qual foi realizado um link entre estas duas linguagens.

A matriz Lagrangeana não deve ser construída diretamente, pois sua dimensão geralmente é muito elevada e uma grande parte de seus elementos são nulos, por esta razão a rotina lagrangeana.c calcula e armazena somente os elementos diferentes de zero e em formato vetorial e para isto utiliza a rotina topologia.c, pois a estrutura da matriz Lagrangeana é dependente da topologia da rede.

Nos trabalhos publicados por GRANVILLE (1994), WU et al. (1994), QUINTANA et al. (1995) e IRISSARI et al. (1997), a matriz Lagrangeana é rearranjada em estruturas de blocos $2 \times 2$ ou $4 \times 4$, os quais estão associados com as barras do sistema de potência, neste caso a matriz de blocos resultante tem a mesma topologia da matriz Jacobiana, definida em (4.5), permitindo desta forma o uso de técnicas de esparsidade, porém a estrutura em blocos não permite o total aproveitamento da esparsidade da matriz, como demonstrou LANGONA (1994). Por esta razão, este trabalho tem como contribuição o processo de solução do sistema (4.25) através de elementos, ao invés de estruturas em blocos.

A solução do sistema (4.25) foi realizada através das rotinas em Fortran. A seguir será apresentado um resumo sobre estas rotinas.

\section{7) ROTINAS UTILIZADAS NA SOLUÇÃO DO SISTEMA}

No algoritmo apresentado anteriormente, utilizou-se para resolver o sistema de equações dadas em (4.25) um programa elaborado em Fortran pelo Grupo de Algoritmos Numéricos do Laboratório Harwell do United Kingdom Atomic Energy Authority. Este programa é utilizado para resolver problemas lineares esparsos 
utilizando uma variante da Eliminação de Gauss. Para utilizar estas rotinas já elaboradas, deve-se criar três vetores:

- vetor a:

o qual receberá somente os valores não nulos da matriz Lagrangeana.

- vetor irn:

o qual receberá o índice da linha do elemento não nulo da matriz Lagrangeana.

- vetor icn:

o qual receberá o índice da coluna do elemento não nulo da matriz Lagrangeana.

Para melhor compreender, considere o seguinte sistema exemplo a seguir:

$$
\left[\begin{array}{ccc}
2.0 & -1.0 & 0.0 \\
-1.0 & 2.0 & 0.5 \\
0.0 & 0.0 & 1.0
\end{array}\right]\left[\begin{array}{l}
x_{1} \\
x_{2} \\
x_{3}
\end{array}\right]=\left[\begin{array}{l}
0.0 \\
0.0 \\
1.0
\end{array}\right]
$$

Para este caso exemplo, os vetores a, irn e icn são os seguintes:

TABELA 2 - Sistema exemplo

\begin{tabular}{|c|c|c|}
\hline a & irn & icn \\
\hline 2.0 & 1 & 1 \\
\hline-1.0 & 2 & 1 \\
\hline-1.0 & 1 & 2 \\
\hline 2.0 & 2 & 2 \\
\hline 0.5 & 2 & 3 \\
\hline 1.0 & 3 & 3 \\
\hline
\end{tabular}

Para obter a solução de (4.33), a rotina em Fortran realiza a seguinte operação, conforme o GUIA SIMPLIFICADO PARA USO DAS SUBROTINAS DO LABORATÓRIO HARWELL NA SOLUÇÃO DE SISTEMAS LINEARES $\mathrm{Ax}=\mathrm{b}$ descreve: 
$P A Q=L U$

onde:

A é uma matriz esparsa;

P e Q são matrizes de permutação;

L é uma matriz triangular inferior unitária;

U uma matriz triangular superior.

Uma vez realizadas estas operações pela rotina ma28ad.f, a solução do sistema de equações é obtida processando-se a rotina ma28cd.f.

\section{8) MATRIZ LAGRANGEANA}

Quando o algoritmo do método dos pontos interiores vai se aproximando da solução do problema original, alguma variável canalizada pode atingir um de seus limites, fazendo com que a variável de folga correspondente se torne muito pequena e por isso o elemento da diagonal principal referente a esta variável se torna muito elevado devido o termo $1 / \mathrm{s}_{\mathrm{i}}(\mathrm{i}=1,2,3,4)$. Apesar do valor de $\mu$ ser pequeno, o termo $\left(\mu 1 / \mathrm{s}_{\mathrm{i}}\right.$ ) pode contribuir grandemente para o mal condicionamento da matriz, causando dificuldades numéricas para o algoritmo.

Com o objetivo de evitar que o algoritmo divirja devido ao mal condicionamento da matriz, as restrições do problema de fluxo da carga ótimo podem ser consideradas suficientemente satisfeitas com tolerância não muito pequena, desde que os problemas nesta área da engenharia não exigem um grau de exatidão tão elevado. 
CAPÍTULO 5

\section{TESTES E RESULTADOS}

\section{1) INTRODUÇÃO}

Neste capítulo serão mostrados os resultados obtidos da aplicação do método dos pontos interiores para solução do problema de fluxo de carga ótimo. Os sistemas elétricos de potência, utilizados para verificar a eficiência do método, foram de 3,14, 30 e 118 barras. Para cada sistema a função objetivo a ser minimizada foi a de perdas de potência ativa na transmissão. A seguir tem-se os quatro sistemas exemplos:

\section{2) SISTEMA DE 3 BARRAS}

O sistema de 3 barras utilizado por DOMMEL \& TINNEY (1968), está mostrado na figura 3 , e tem as seguintes características:

- 1 barra de referência;

- 1 barra de controle de reativo;

- 1 barra de carga;

- 2 linhas de transmissão. 


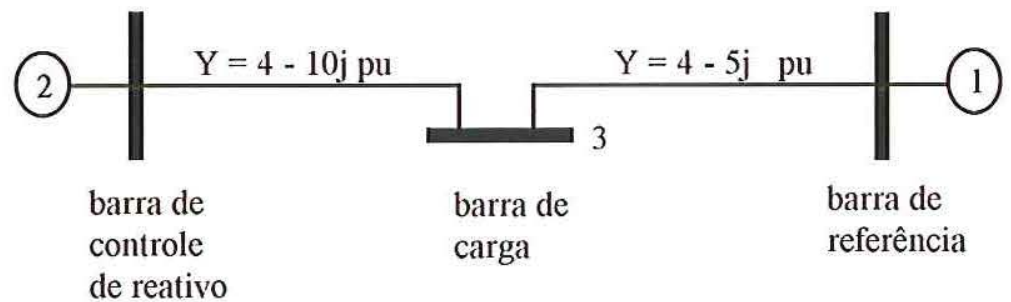

FIGURA 3 - Sistema elétrico de 3 barras

O problema de fluxo de carga ótimo para este sistema de 3 barras pode ser escrito da seguinte forma:

$\min \mathrm{g}_{23}\left(\mathrm{~V}_{2}^{2}+\mathrm{V}_{3}^{2}-2 \mathrm{~V}_{2} \mathrm{~V}_{3} \cos \theta_{23}\right)+\mathrm{g}_{31}\left(\mathrm{~V}_{3}^{2}+\mathrm{V}_{1}^{2}-2 \mathrm{~V}_{3} \mathrm{~V}_{1} \cos \theta_{31}\right)$

s. a $\quad \Delta P_{2}=P_{2}^{G}-P_{2}^{C}-V_{2} \sum_{i=1}^{3} V_{i}\left(G_{2 i} \cos \theta_{2 i}+B_{2 i} \operatorname{sen} \theta_{2 i}\right)=0$

$\Delta P_{3}=P_{3}^{G}-P_{3}^{C}-V_{3} \sum_{i=1}^{3} V_{i}\left(G_{3 i} \cos \theta_{3 i}+B_{3 i} \operatorname{sen} \theta_{3 i}\right)=0$

$\Delta Q_{3}=Q_{3}^{G}-Q_{3}^{C}-V_{3} \sum_{i=1}^{3} V_{i}\left(G_{3 i} \operatorname{sen} \theta_{3 i}-B_{3 i} \cos \theta_{3 i}\right)=0$

$\mathrm{V}_{1}^{\min } \leq \mathrm{V}_{1} \leq \mathrm{V}_{1}^{\max }$

$\mathrm{V}_{2}^{\min } \leq \mathrm{V}_{2} \leq \mathrm{V}_{2}^{\max }$

$\mathrm{V}_{3}=1.00 \mathrm{pu}$ 
onde:

$$
\begin{array}{ll}
\mathrm{P}_{2}^{\mathrm{G}}=170 \mathrm{MW} & \mathrm{P}_{3}^{\mathrm{G}}=0 \mathrm{MW} \\
\mathrm{P}_{2}^{\mathrm{C}}=0 \mathrm{MW} & \mathrm{P}_{3}^{\mathrm{C}}=200 \mathrm{MW} \\
\mathrm{Q}_{3}^{\mathrm{G}}=0 \mathrm{M} \text { var } & \mathrm{Q}_{3}^{\mathrm{C}}=100 \mathrm{M} \text { var } \\
\mathrm{V}_{1}^{\min }=1.01 \mathrm{pu} & \mathrm{V}_{1}^{\max }=1.10 \mathrm{pu} \\
\mathrm{V}_{2}^{\min }=1.01 \mathrm{pu} & \mathrm{V}_{2}^{\max }=1.20 \mathrm{pu}
\end{array}
$$

Colocando o problema (5.1) na forma apropriada para aplicação do método primal-dual barreira-logarítmica, pode-se escrever então a função Lagrangeana, a qual é dada por:

$$
\begin{aligned}
L= & g_{23}\left(V_{2}^{2}+V_{3}^{2}-2 V_{2} V_{3} \cos \theta_{23}\right)+g_{31}\left(V_{3}^{2}+V_{1}^{2}-2 V_{3} V_{1} \cos \theta_{31}\right)- \\
& -\lambda p_{2}\left(P_{2}^{G}-P_{2}^{C}-V_{2} \sum_{i=1}^{3} V_{i}\left(G_{2 i} \cos \theta_{2 i}+B_{2 i} \operatorname{sen} \theta_{2 i}\right)\right)- \\
& -\lambda p_{3}\left(P_{3}^{G}-P_{3}^{C}-V_{3} \sum_{i=1}^{3} V_{i}\left(G_{3 i} \cos \theta_{3 i}+B_{3 i} \operatorname{sen} \theta_{3 i}\right)\right)- \\
& -\lambda q_{3}\left(Q_{3}^{G}-Q_{3}^{C}-V_{3} \sum_{i=1}^{3} V_{i}\left(G_{3 i} \operatorname{sen} \theta_{3 i}-B_{3 i} \cos \theta_{3 i}\right)\right)- \\
& -\pi_{31}\left(V_{1}+s_{31}-V_{1}^{\max }\right)-\pi_{32}\left(V_{2}+s_{32}-V_{2}^{\max }\right)- \\
& -\pi_{41}\left(V_{1}-s_{41}-V_{1}^{\min }\right)-\pi_{42}\left(V_{2}-s_{42}-V_{2}^{\min }\right)- \\
& -\mu \ln \left(s_{31}\right)-\mu \ln \left(s_{32}\right)-\mu \ln \left(s_{41}\right)-\mu \ln \left(s_{42}\right)
\end{aligned}
$$

A tabela 3 a seguir, tem por objetivo apresentar detalhadamente o processo de convergência do método, onde será apresentado o valor de todas as variáveis utilizadas. Considerou-se $\beta=1,2$ para atualização do parâmetro de barreira $\mu$, e este foi atualizado por (4.32). 
TABELA 3 - Valores das variáveis utilizadas nos cálculos

\section{Estado inicial do sistema}

\begin{tabular}{|c|c|c|c|}
\hline \multicolumn{5}{|c|}{ tensões e ângulos } \\
\hline \hline Barra & Tipo & V (p.u.) & ang (grau) \\
\hline 1 & SL & 1,0000 & 0,0000 \\
\hline 2 & CR & 1,0000 & $-2,0000$ \\
\hline 3 & CG & 1,0000 & $-5,0000$ \\
\hline
\end{tabular}

\begin{tabular}{|c|c|c|c|c|}
\hline \hline \multicolumn{5}{|c||}{ multiplicadores de Lagrange } \\
\hline \hline Barra & $\lambda p$ & $\lambda q$ & $\pi_{3}$ & $\pi_{4}$ \\
\hline 1 & - & - & $-0,0010$ & 0,0050 \\
\hline 2 & 1,0000 & - & $-0,0005$ & 0,0050 \\
\hline 3 & 1,0000 & 1,0000 & - & - \\
\hline
\end{tabular}

\begin{tabular}{|c|c|c|}
\hline \multicolumn{3}{|c|}{ variáveis de folga } \\
\hline \hline Barra & $\mathrm{s}_{3}$ & $\mathrm{~s}_{4}$ \\
\hline 1 & 0,1000 & 0,0200 \\
\hline 2 & 0,2000 & 0,0200 \\
\hline 3 & - & - \\
\hline
\end{tabular}

parâmetro de barreira, função objetivo e restrições de igualdade

\begin{tabular}{|c|c|c|c|c|}
\hline \hline$\mu$ & f.o. (p.u.) & $\Delta \mathrm{P}_{2}$ (p.u.) & $\Delta \mathrm{P}_{3}$ (p.u.) & $\Delta \mathrm{Q}_{3}$ (p.u.) \\
\hline 0,0001 & 0,0414 & $-1,1712$ & 1,0616 & 1,5910 \\
\hline
\end{tabular}


Iteração 1

tensões e ângulos

\begin{tabular}{|c|c|c|c|}
\hline \hline Barra & Tipo & V (p.u.) & ang (grau) \\
\hline 1 & SL & 1,0570 & 0,0000 \\
\hline 2 & CR & 1,1293 & 5,4463 \\
\hline 3 & CG & 1,0000 & $-0,8413$ \\
\hline
\end{tabular}

multiplicadores de Lagrange

\begin{tabular}{|c|c|c|c|c|}
\hline \hline Barra & $\lambda p$ & $\lambda q$ & $\pi_{3}$ & $\pi_{4}$ \\
\hline 1 & - & - & $-0,0011$ & 0,0036 \\
\hline 2 & 0,7364 & - & $-0,0006$ & 0,0000 \\
\hline 3 & 0,7970 & 0,8203 & - & - \\
\hline
\end{tabular}

variáveis de folga

\begin{tabular}{|c|c|c|}
\hline \hline Barra & $\mathrm{s}_{3}$ & $\mathrm{~s}_{4}$ \\
\hline 1 & 0,0430 & 0,0471 \\
\hline 2 & 0,0707 & 0,1193 \\
\hline 3 & - & - \\
\hline
\end{tabular}

\begin{tabular}{|c|c|c|c|c||}
\hline \multicolumn{5}{|c||}{ parâmetro de barreira, função objetivo e restrições de igualdade } \\
\hline \hline$\mu$ & f.o. (p.u.) & $\Delta \mathrm{P}_{2}$ (p.u.) & $\Delta \mathrm{P}_{3}$ (p.u.) & $\Delta \mathrm{Q}_{3}$ (p.u.) \\
\hline 0,00008333 & 0,1351 & 0,1480 & $-0,0321$ & 0,0470 \\
\hline
\end{tabular}

\begin{tabular}{|c|c|}
\hline \multicolumn{2}{|c|}{ tamanho dos passos } \\
\hline \hline$\alpha_{\mathrm{p}}$ & $\alpha_{\mathrm{d}}$ \\
\hline 1,0000 & 0,2013 \\
\hline
\end{tabular}




\section{Iteração 2}

\section{tensões e ângulos}

\begin{tabular}{|c|c|c|c|}
\hline \hline Barra & Tipo & V (p.u.) & ang (grau) \\
\hline 1 & SL & 1,0731 & 0,0000 \\
\hline 2 & CR & 1,1232 & 4,5259 \\
\hline 3 & CG & 1,0000 & $-1,2209$ \\
\hline
\end{tabular}

\begin{tabular}{|c|c|c|c|c|}
\hline \hline \multicolumn{5}{|c||}{ multiplicadores de Lagrange } \\
\hline \hline Barra & $\lambda p$ & $\lambda q$ & $\pi_{3}$ & $\pi_{4}$ \\
\hline 1 & - & - & $-0,0027$ & 0,0012 \\
\hline 2 & $-0,0091$ & - & $-0,0011$ & 0,0007 \\
\hline 3 & 0,0839 & 0,0497 & - & - \\
\hline
\end{tabular}

\begin{tabular}{|c|c|c|}
\hline \multicolumn{3}{|c|}{ variáveis de folga } \\
\hline \hline Barra & $\mathrm{s}_{3}$ & $\mathrm{~s}_{4}$ \\
\hline 1 & 0,0269 & 0,0631 \\
\hline 2 & 0,0768 & 0,1132 \\
\hline 3 & - & - \\
\hline
\end{tabular}

\begin{tabular}{|c|c|c|c|c||}
\hline \multicolumn{5}{|c||}{ parâmetro de barreira, função objetivo e restrições de igualdade } \\
\hline \hline$\mu$ & f.o. (p.u.) & $\Delta \mathrm{P}_{2}$ (p.u.) & $\Delta \mathrm{P}_{3}$ (p.u.) & $\Delta \mathrm{Q}_{3}$ (p.u.) \\
\hline 0,0000694 & 0,1292 & 0,0010 & $-0,0007$ & 0,0010 \\
\hline
\end{tabular}

\begin{tabular}{|c|c|}
\hline \multicolumn{2}{|c||}{ tamanho dos passos } \\
\hline \hline$\alpha_{\mathrm{p}}$ & $\alpha_{\mathrm{d}}$ \\
\hline 1,0000 & 1,0000 \\
\hline
\end{tabular}


Iteração 3

tensões e ângulos

\begin{tabular}{|c|c|c|c|}
\hline \hline Barra & Tipo & V (p.u.) & ang (grau) \\
\hline 1 & SL & 1,0711 & 0,0000 \\
\hline 2 & CR & 1,1247 & 4,3794 \\
\hline 3 & CG & 1,0000 & $-1,3195$ \\
\hline
\end{tabular}

multiplicadores de Lagrange

\begin{tabular}{|c|c|c|c|c|}
\hline \hline Barra & $\lambda p$ & $\lambda q$ & $\pi_{3}$ & $\pi_{4}$ \\
\hline 1 & - & - & $-0,0024$ & 0,0011 \\
\hline 2 & $-0,0274$ & - & $-0,0009$ & 0,0006 \\
\hline 3 & 0,0795 & 0,0500 & - & - \\
\hline
\end{tabular}

variáveis de folga

\begin{tabular}{|c|c|c|}
\hline \hline Barra & $\mathrm{s}_{3}$ & $\mathrm{~s}_{4}$ \\
\hline 1 & 0,0289 & 0,0611 \\
\hline 2 & 0,0753 & 0,1147 \\
\hline 3 & - & - \\
\hline
\end{tabular}

\begin{tabular}{|c|c|c|c|c||}
\hline \multicolumn{5}{|c||}{ parâmetro de barreira, função objetivo e restrições de igualdade } \\
\hline \hline$\mu$ & f.o. (p.u.) & $\Delta \mathrm{P}_{2}$ (p.u.) & $\Delta \mathrm{P}_{3}$ (p.u.) & $\Delta \mathrm{Q}_{3}$ (p.u.) \\
\hline 0,0000579 & 0,1292 & 0,0000 & 0,0000 & 0,0000 \\
\hline
\end{tabular}

\begin{tabular}{|c|c|}
\hline \multicolumn{2}{|c|}{ tamanho dos passos } \\
\hline \hline$\alpha_{p}$ & $\alpha_{d}$ \\
\hline 1,0000 & 1,0000 \\
\hline
\end{tabular}

Perdas ativas do sistema $=12,9153 \mathrm{MW}$ 
Como pôde ser observado na tabela 3 , as tensões $V_{1}$ e $V_{2}$ tenderam para o seus limites superiores, ou seja:

$$
\mathrm{V}_{1} \rightarrow \mathrm{V}_{1}^{\max } \quad \mathrm{V}_{2} \rightarrow \mathrm{V}_{2}^{\max }
$$

Desta forma teve-se que os elementos do vetor das variáveis de folga $s_{3}$, foram menores do que os elementos de $s_{4}$, porém como nenhuma tensão atingiu seu limite, as variáveis de folga assumiram valores diferentes de zero. Os multiplicadores de Lagrange satisfizeram as condições de KKT, pois como as restrições de desigualdade tendem a ser ativas pelo máximo, e inativas pelo mínimo, teve-se que $\pi_{3}<0$ e $\pi_{4} \rightarrow 0$, que são os multiplicadores em relação ao máximo e mínimo respectivamente.

O processo convergiu em 3 iterações com um tempo de CPU igual a 0,28 segundos e vetor gradiente nulo. O ponto de operação obtido pelo FCO desenvolvido atingiu a mesma solução dos métodos propostos por DOMMEL \& TINNEY (1968) e LANGONA (1994). A figura 4 mostra o gráfico da função objetivo para o sistema de 3 barras.

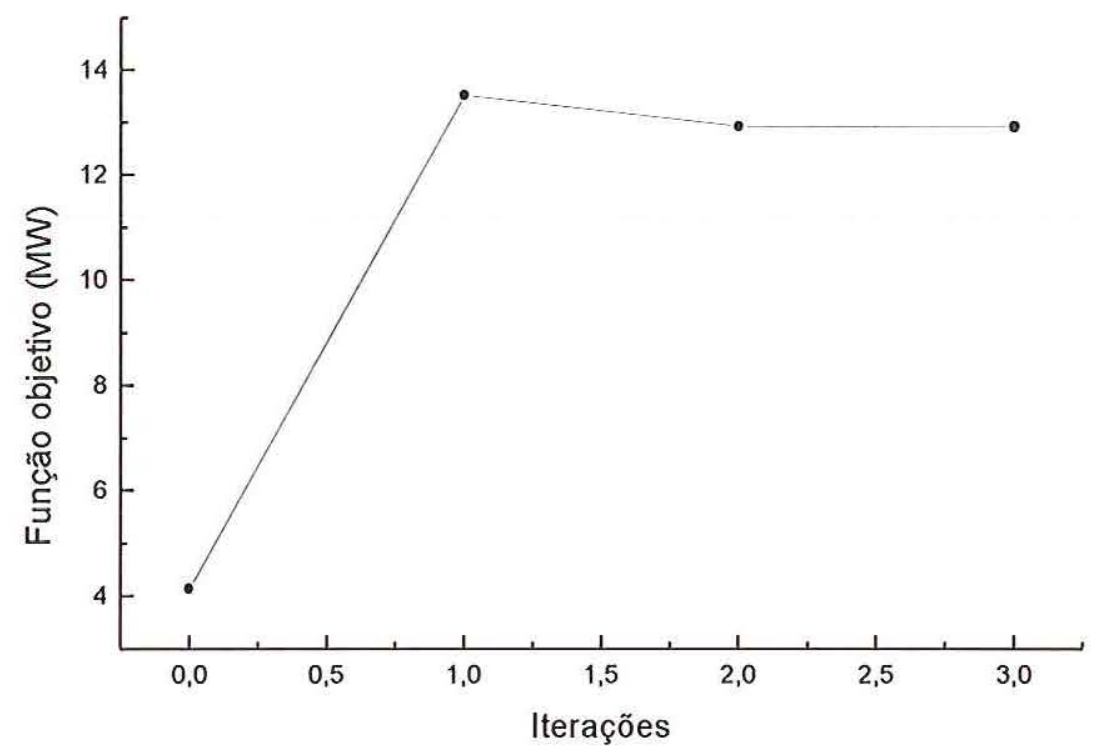

FIGURA 4 - Função objetivo do sistema de 3 barras 
A figura 5 mostra a trajetória seguida pelas variáveis $V_{1} e V_{2}$, a cada iteração.

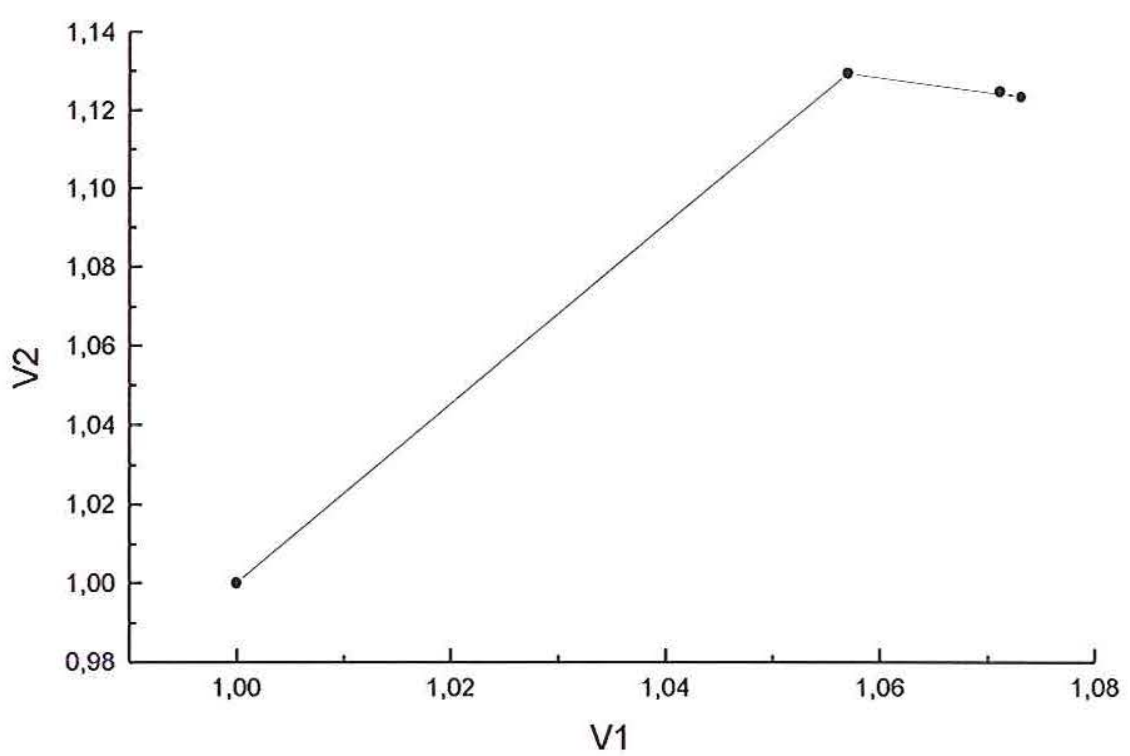

FIGURA 5 - Convergência de $V_{1}$ e $V_{2}$ para o sistema de 3 barras (p.u.)

\section{3) SISTEMA DE 14 BARRAS}

O sistema de 14 barras, obtido em FRERIS \& SASSON (1968), está mostrado na figura 6 , e tem as seguintes características:

- 1 barra de referência;

- 4 barras de controle de reativo;

- 9 barras de carga;

- 17 linhas de transmissão;

- 3 transformadores com tap variável. 


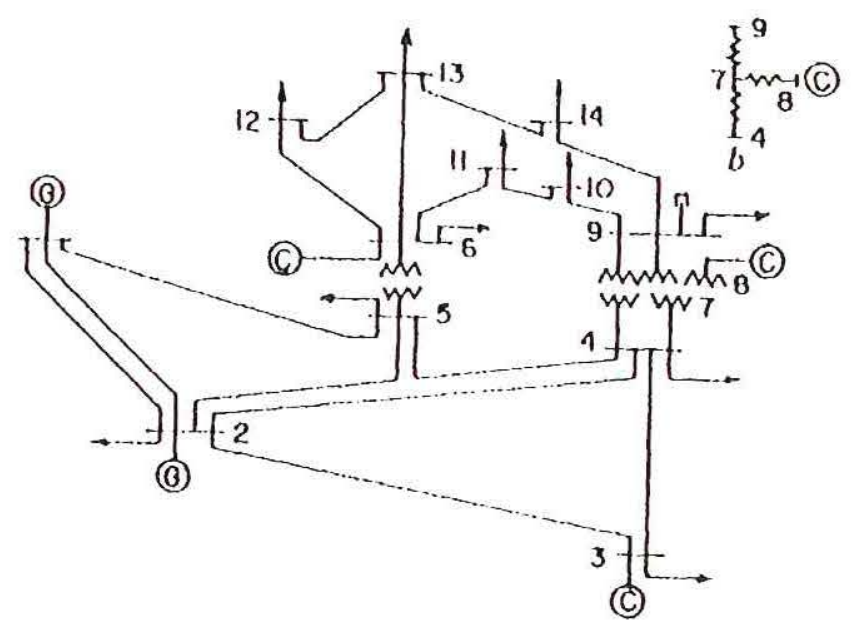

(C) COMPENSADORES SINCRONOS

(G) GERADORES

FIGURA 6 - Sistema elétrico de 14 barras

A função Lagrangeana para o problema de minimização do sistema de 14 barras é dada por:

$$
\begin{aligned}
L= & f(x)-\lambda^{t} g(x)-\pi_{1}\left(Q(x)+s_{1}-Q^{\max }\right)-\pi_{2}\left(Q(x)-s_{2}-Q^{m i n}\right)- \\
& -\pi_{3}\left(V+s_{3}-V^{\max }\right)-\pi_{4}\left(V-s_{4}-V^{\min }\right)- \\
& -\pi_{5}\left(t+s_{5}-t^{\max }\right)-\pi_{6}\left(t-s_{6}-t^{\min }\right)- \\
& -\mu \sum_{i=1}^{4} \ln \left(s_{1 i}\right)-\mu \sum_{i=1}^{4} \ln \left(s_{2 i}\right)-\mu \sum_{i=1}^{14} \ln \left(s_{3 i}\right)-\mu \sum_{i=1}^{14} \ln \left(s_{4 i}\right) \\
& -\mu_{t} \sum_{i=1}^{3} \ln \left(s_{5 i}\right)-\mu_{t} \sum_{i=1}^{3} \ln \left(s_{6 i}\right)
\end{aligned}
$$

onde:

$\mathrm{Q}(\mathrm{x})$ é calculado através de (4.4);

t é o tap do transformador;

$\mu_{t}$ parâmetro de barreira usado na função barreira logarítmica em relação as variáveis de folga do tap. 
Testes foram realizados e verificou-se que o método primal-dual barreiralogarítmica é muito sensível quanto aos valores atribuídos para os parâmetros $\mu$ e $\beta$. A tabela 4 a seguir, mostra os valores iniciais atribuídos para as variáveis $\mu$ e $\mu_{\mathrm{t}}$, os valores dos parâmetros $\beta$ e $\beta_{1}$ mantidos constantes durante todo o processo iterativo, o número de iterações e o valor da função objetivo após a convergência. Esta tabela tem como objetivo mostrar a sensibilidade do método quanto ao parâmetro de barreira, pois para este caso estudado, não variamos os valores dos parâmetros $\beta$ e $\beta_{\mathrm{t}}$, utilizados na atualização de $\mu \mathrm{e} \mu_{\mathrm{t}}$, respectivamente.

TABELA 4 - Testes para o sistema de 14 barras

\begin{tabular}{|c|c|c|c|c|c|c|}
\hline caso & $\begin{array}{c}\mu \\
\text { inicial }\end{array}$ & $\beta$ & $\begin{array}{c}\mu_{\mathrm{t}} \\
\text { inicial }\end{array}$ & $\beta_{\mathrm{t}}$ & iterações & $\begin{array}{c}\text { perdas ativas do } \\
\text { sistema (MW) }\end{array}$ \\
\hline 1 & 0,1 & 1,01 & 0,1 & 1,01 & 4 & 13,2326 \\
\hline 2 & 0,01 & 1,01 & 0,01 & 1,01 & 4 & 13,0965 \\
\hline $3^{*}$ & 0,001 & 1,01 & 0,01 & 1,01 & 4 & 12,4620 \\
\hline 4 & 0,1 & 1,01 & 1 & 1,01 & 4 & 13,0925 \\
\hline 5 & 1 & 1,01 & 0,1 & 1,01 & 4 & 13,3973 \\
\hline
\end{tabular}

Observe que para cada caso realizado, o valor das perdas de potência ativas do sistema foi diferente, ou seja, cada caso forneceu um ponto de operação para o sistema, o qual satisfez todas as restrições de operação. Note que nos dois primeiros casos, utilizou-se o mesmo valor para os parâmetros de barreira $\mu$ e $\mu_{\mathrm{t}}$, já para o caso 3 em diante, utilizou-se valores diferentes, sendo que o caso 3 foi o que apresentou o menor valor de função objetivo. Por esta razão, na função Lagrangeana dada em (5.3), escreveu-se $\mu_{t}$ na função barreira logarítmica das variáveis de folga em relação ao tap do transformador.

O estado inicial e final do sistema para o caso 3 , poderá ser visto nos Apêndices 1 e 2, respectivamente. Os gráficos a cada iteração das restrições de igualdade $\Delta \mathrm{P}$ e $\Delta \mathrm{Q}$ (Mismatches) e da função objetivo, são mostrados nas figuras $7 \mathrm{e}$ 8 respectivamente. 


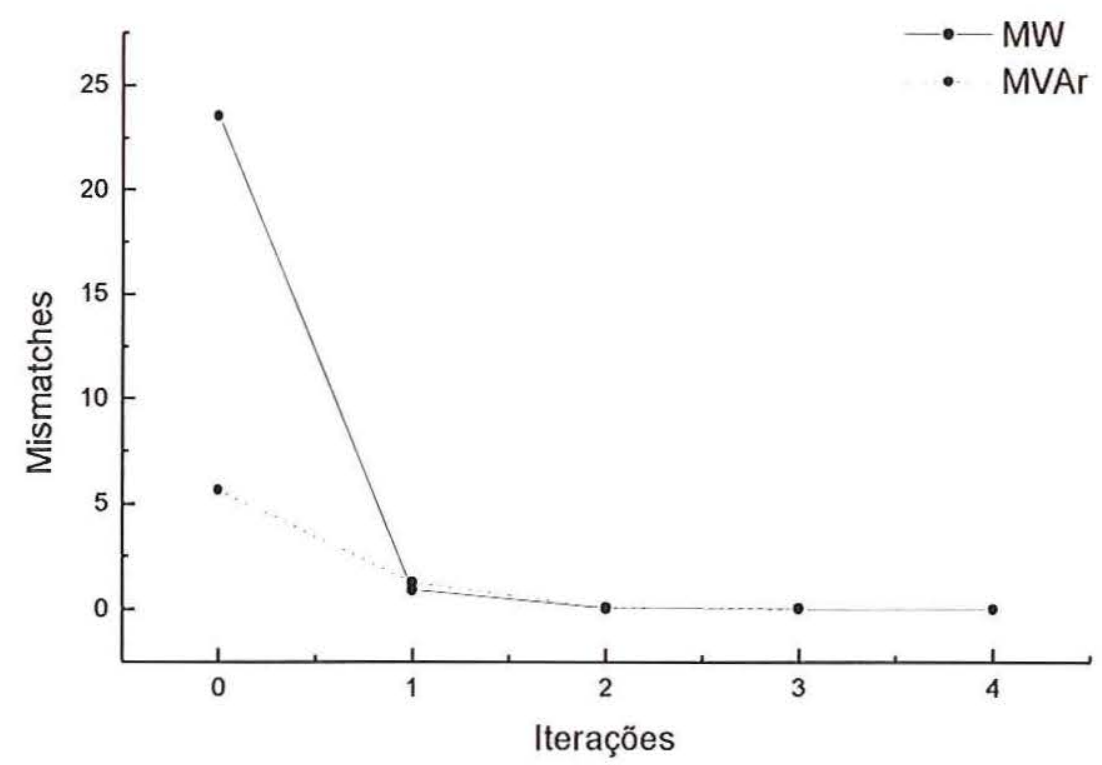

FIGURA 7 - As restrições $\Delta \mathrm{P}$ e $\Delta \mathrm{Q}$ a cada iteração

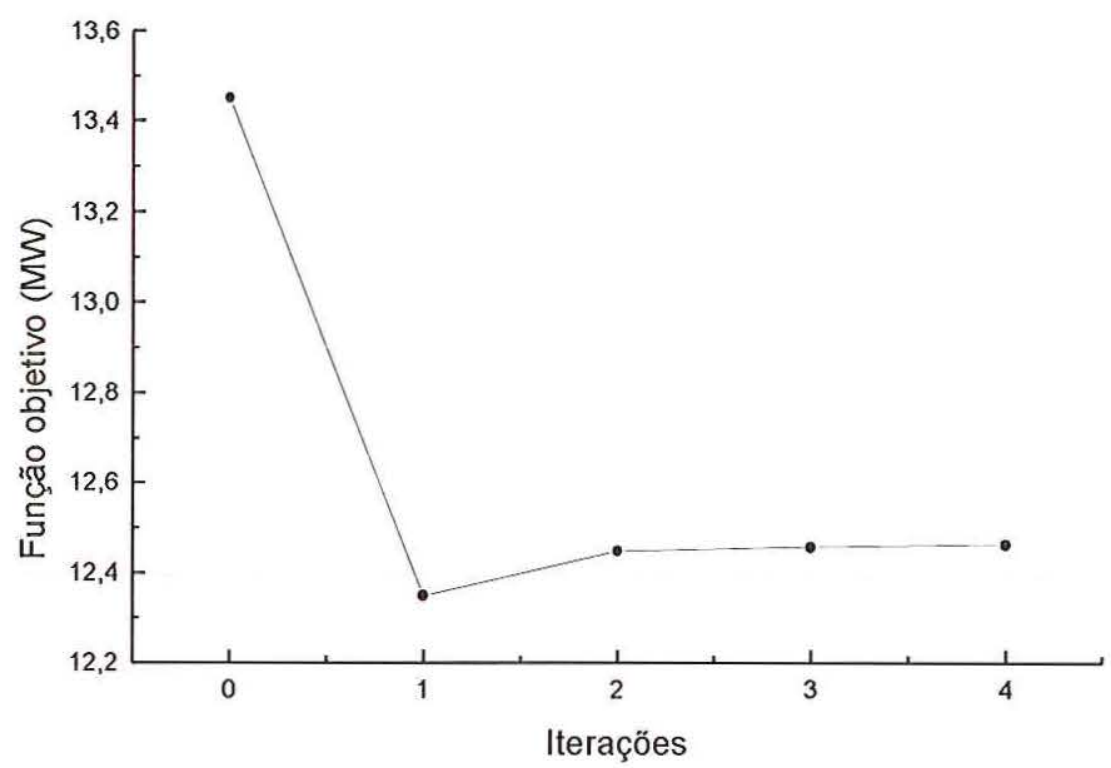

FIGURA 8 - Função objetivo

Como pôde ser observado pelos gráficos anteriores, o processo convergiu em 4 iterações atingindo um valor final de função objetivo igual a 12,4620 MW. O tempo de CPU foi de 0,58 segundos e o vetor gradiente nulo, satisfazendo as condições de otimalidade. 
Através do Apêndice 2, note que a tensão da barra 1 quase atingiu o seu limite superior, fazendo com que a variável de folga associada $\left(\mathrm{s}_{31}\right)$, fosse praticamente nula. Desta forma podemos considerar a restrição de desigualdade $V_{1} \leq V_{1}^{\max }$ ativa pelo máximo na solução, e pelas condições de KKT, teve-se que $\pi_{3}<0$. Como a tensão da barra 1 é inativa pelo mínimo teve-se que $\pi_{4}=0$.

\section{4) SISTEMA DE 30 BARRAS}

O sistema de 30 barras, obtido em FRERIS \& SASSON (1968), está mostrado na figura 9, e tem as seguintes características:

- 1 barra de referência;

- 5 barras de controle de reativo;

- 24 barras de carga;

- 37 linhas de transmissão;

- 4 transformadores com tap variável. 


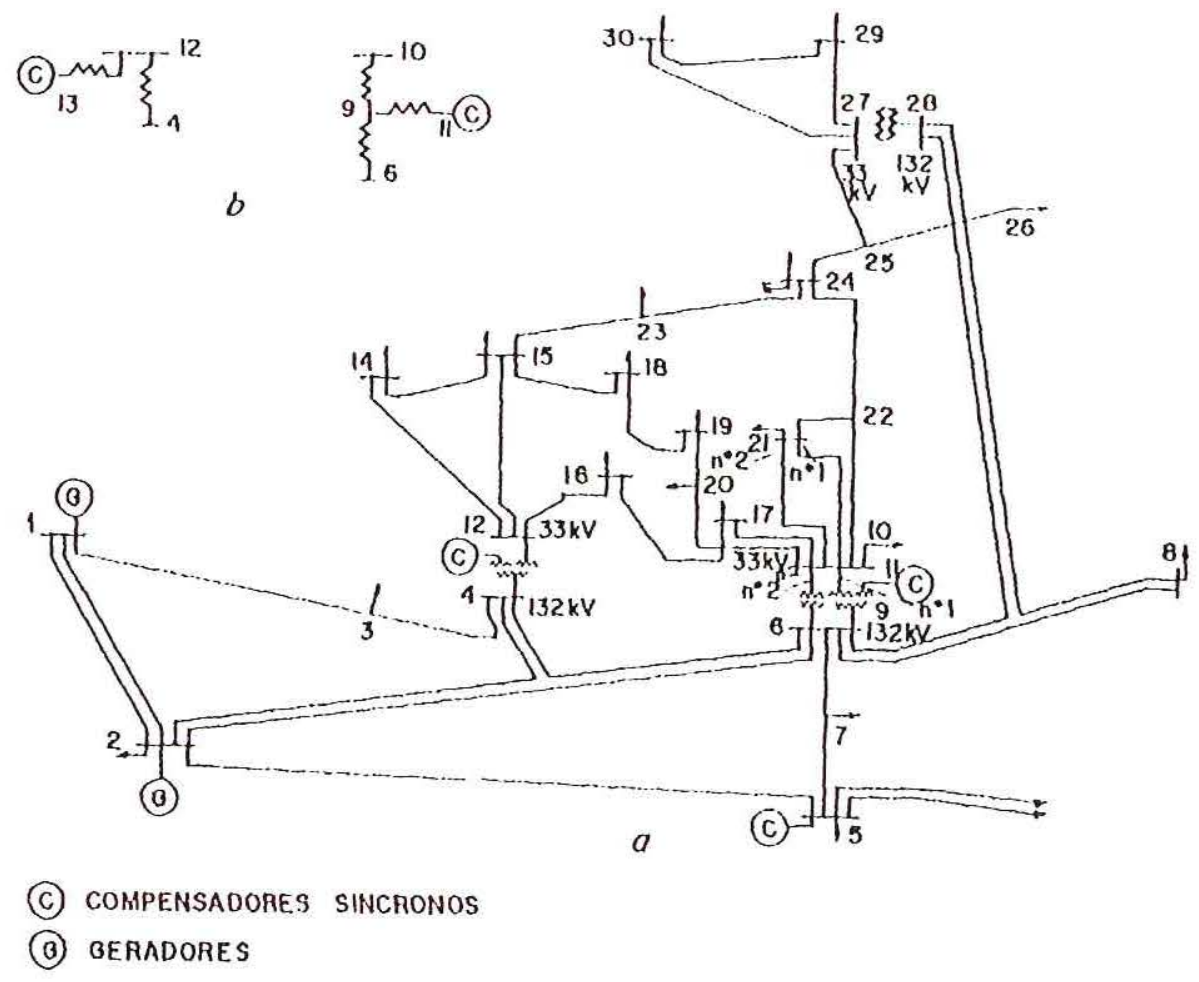

FIGURA 9 - Sistema elétrico de 30 barras

A função Lagrangeana para o problema de minimização do sistema de 30 barras é dada por: 


$$
\begin{aligned}
L= & f(x)-\lambda^{t} g(x)-\pi_{1}\left(Q(x)+s_{1}-Q^{\max }\right)-\pi_{2}\left(Q(x)-s_{2}-Q^{\min }\right)- \\
& -\pi_{3}\left(V+s_{3}-V^{\max }\right)-\pi_{4}\left(V-s_{4}-V^{\min }\right)- \\
& -\pi_{5}\left(t+s_{5}-t^{\max }\right)-\pi_{6}\left(t-s_{6}-t^{\min }\right)- \\
& -\mu \sum_{i=1}^{5} \ln \left(s_{1 i}\right)-\mu \sum_{i=1}^{5} \ln \left(s_{2 i}\right)-\mu \sum_{i=1}^{30} \ln \left(s_{3 i}\right)-\mu \sum_{i=1}^{30} \ln \left(s_{4 i}\right) \\
& -\mu_{t} \sum_{i=1}^{4} \ln \left(s_{5 i}\right)-\mu_{t} \sum_{i=1}^{4} \ln \left(s_{6 i}\right)
\end{aligned}
$$

onde:

$\mathrm{Q}(\mathrm{x})$ é calculado através de (4.4);

t é o tap do transformador;

$\mu_{\mathrm{t}}$ parâmetro de barreira usado na função barreira logarítmica em relação as variáveis de folga do tap.

Para o sistema de 30 barras, além de se variar os valores atribuídos para $\mu \mathrm{e}$ $\mu_{\mathrm{t}}$, variou-se também os valores de $\beta$ e $\beta_{\mathrm{t}}$, utilizados na atualização destes parâmetros. A tabela 5 mostra os valores iniciais atribuídos para as variáveis $\mu \mathrm{e} \mu_{\mathrm{t}}$, os valores dos parâmetros $\beta$ e $\beta_{1}$ mantidos constantes durante todo o processo iterativo, o número de iterações e o valor da função objetivo após a convergência. Esta tabela mostra a sensibilidade do método quanto a estes parâmetros.

TABELA 5 - Testes para o sistema de 30 barras

\begin{tabular}{|c|c|c|c|c|c|c|}
\hline caso & $\begin{array}{c}\mu \\
\text { inicial }\end{array}$ & $\beta$ & $\begin{array}{c}\mu_{\mathbf{t}} \\
\text { inicial }\end{array}$ & $\beta_{\mathbf{t}}$ & iterações & $\begin{array}{c}\text { perdas ativas do } \\
\text { sistema (MW) }\end{array}$ \\
\hline 1 & 0,001 & 1,01 & 1 & 1,01 & 5 & 16,4265 \\
\hline $2^{*}$ & 0,001 & 1,2 & 1 & 1,1 & 5 & 16,3564 \\
\hline 3 & 0,001 & 1,05 & 1 & 1,05 & 5 & 16,4108 \\
\hline 4 & 1 & 1,01 & 1 & 1,01 & 5 & 16,9970 \\
\hline 5 & 1 & 1,01 & 5 & 1,2 & 5 & 16,9052 \\
\hline
\end{tabular}

Note que nos três primeiros casos, consideramos os valores de $\mu$ e $\mu_{1}$ fixos e variamos somente os valores de $\beta$ e $\beta_{\mathrm{t}}$. Além destes casos, outros testes foram realizados obtendo-se também diferentes valores de perdas ativas para o sistema. 
O estado inicial e final do sistema para o caso 2 , poderá ser visto nos Apêndices 1 e 2, respectivamente. Os gráficos a cada iteração das restrições de igualdade $\Delta \mathrm{P}$ e $\Delta \mathrm{Q}$ (Mismatches) e da função objetivo, são mostrados nas figuras 10 e 11 respectivamente.

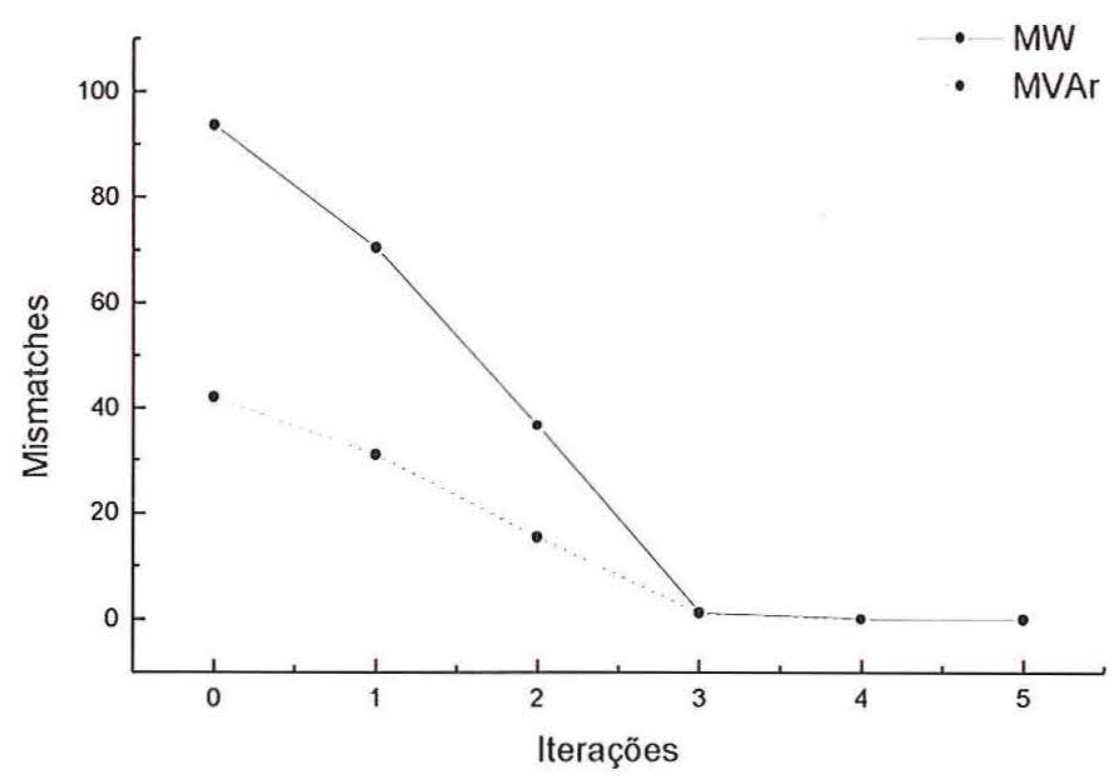

FIGURA 10 - As restrições $\Delta \mathrm{P}$ e $\Delta \mathrm{Q}$ a cada iteração

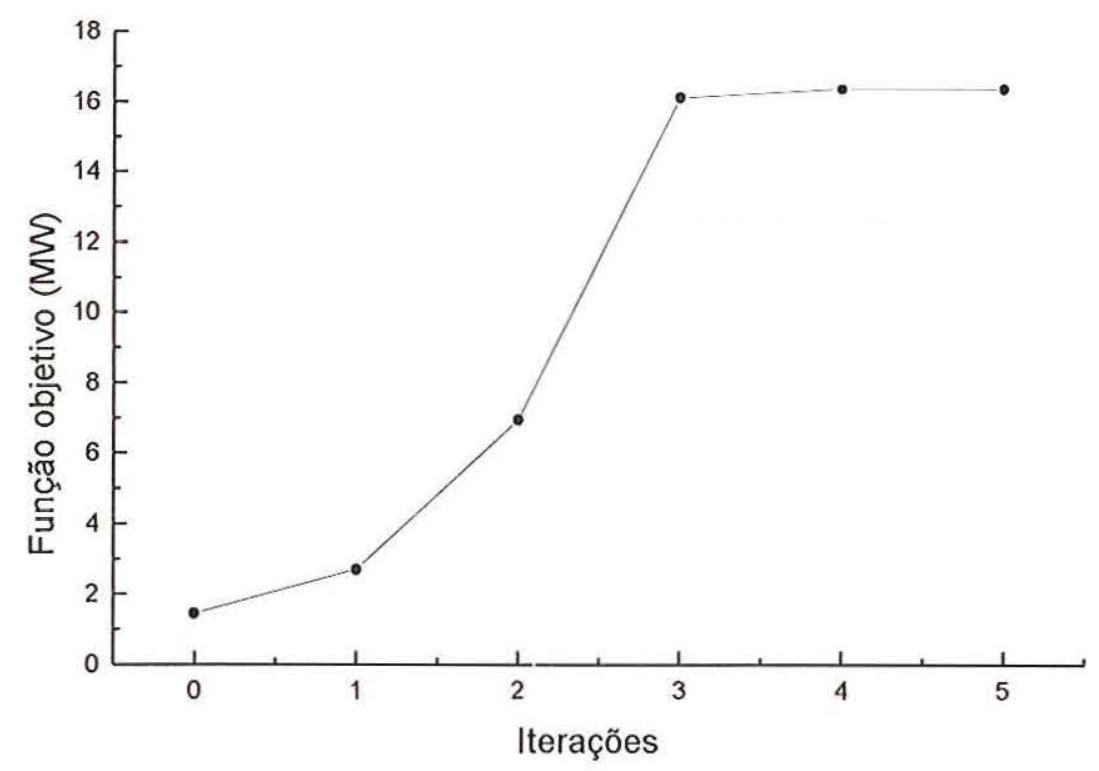

FIGURA 11 - Função objetivo 
Como pôde ser observado pelos gráficos anteriores, o processo convergiu em 5 iterações atingindo um valor final de função objetivo igual a 16,3564 MW. O tempo de CPU foi de 1,14 segundos e o vetor gradiente nulo, satisfazendo as condições de otimalidade.

No Apêndice 2 encontram-se os valores dos multiplicadores de Lagrange associados com as restrições de desigualdade que se tornaram em igualdade pelo uso das variáveis de folga. Através destes pode ser observado que as condições de KKT foram satisfeitas no ponto de solução do problema. Neste sistema também a tensão na barra 1 foi a que mais se aproximou do seu limite superior, podemos então considerar a restrição $V_{1} \leq V_{1}^{\max }$ ativa pelo máximo e inativa pelo mínimo.

\section{5) SISTEMA DE 118 BARRAS}

O sistema de 118 barras tem as seguintes características:

- 1 barra de referência;

- 51 barras de controle de reativo;

- 66 barras de carga;

- 186 linhas de transmissão;

- 9 transformadores com tap variável.

A função Lagrangeana para o problema de minimização do sistema de 118 barras é dada por: 


$$
\begin{aligned}
\mathrm{L}= & \mathrm{f}(\mathrm{x})-\lambda^{\mathrm{t}} \mathrm{g}(\mathrm{x})-\pi_{1}\left(\mathrm{Q}(\mathrm{x})+\mathrm{s}_{1}-\mathrm{Q}^{\mathrm{max}}\right)-\pi_{2}\left(\mathrm{Q}(\mathrm{x})-\mathrm{s}_{2}-\mathrm{Q}^{\mathrm{min}}\right)- \\
& -\pi_{3}\left(\mathrm{~V}+\mathrm{s}_{3}-\mathrm{V}^{\mathrm{max}}\right)-\pi_{4}\left(\mathrm{~V}-\mathrm{s}_{4}-\mathrm{V}^{\mathrm{min}}\right)- \\
& -\pi_{5}\left(\mathrm{t}+\mathrm{s}_{5}-\mathrm{t}^{\mathrm{max}}\right)-\pi_{6}\left(\mathrm{t}-\mathrm{s}_{6}-\mathrm{t}^{\mathrm{min}}\right)- \\
& -\mu \sum_{\mathrm{i}=1}^{51} \ln \left(\mathrm{s}_{1 \mathrm{i}}\right)-\mu \sum_{\mathrm{i}=1}^{51} \ln \left(\mathrm{s}_{2 \mathrm{i}}\right)-\mu \sum_{\mathrm{i}=1}^{118} \ln \left(\mathrm{s}_{3 \mathrm{i}}\right)-\mu \sum_{\mathrm{i}=1}^{118} \ln \left(\mathrm{s}_{4 \mathrm{i}}\right) \\
& -\mu_{\mathrm{t}} \sum_{\mathrm{i}=1}^{9} \ln \left(\mathrm{s}_{5 \mathrm{i}}\right)-\mu_{\mathrm{t}} \sum_{\mathrm{i}=1}^{9} \ln \left(\mathrm{s}_{6 \mathrm{i}}\right)
\end{aligned}
$$

onde:

$\mathrm{Q}(\mathrm{x})$ é calculado através de (4.4);

t é o tap do transformador;

$\mu_{t}$ parâmetro de barreira usado na função barreira logarítmica em relação as variáveis de folga do tap.

O sistema de 118 barras foi considerado o de mais dificil convergência, pois muitos testes foram realizados com o objetivo de encontrar valores para os parâmetros $\mu, \beta, \mu_{\mathrm{t}}$ e $\beta_{\mathrm{t}}$, tais que satisfizessem todas as restrições de operação do sistema. Desta forma o valores atribuídos para estas variáveis, tais que satisfizessem todas as restrições, foram: $\mu=0,001 ; \beta=1,45 ; \mu_{t}=55$ e $\beta_{t}=1,45$.

O estado inicial e final do sistema poderá ser visto nos Apêndices 1 e 2, respectivamente. Os gráficos a cada iteração das restrições de igualdade $\Delta \mathrm{P}$ e $\Delta \mathrm{Q}$ (Mismatches) e da função objetivo, são mostrados nas figuras 12 e 13 respectivamente. 


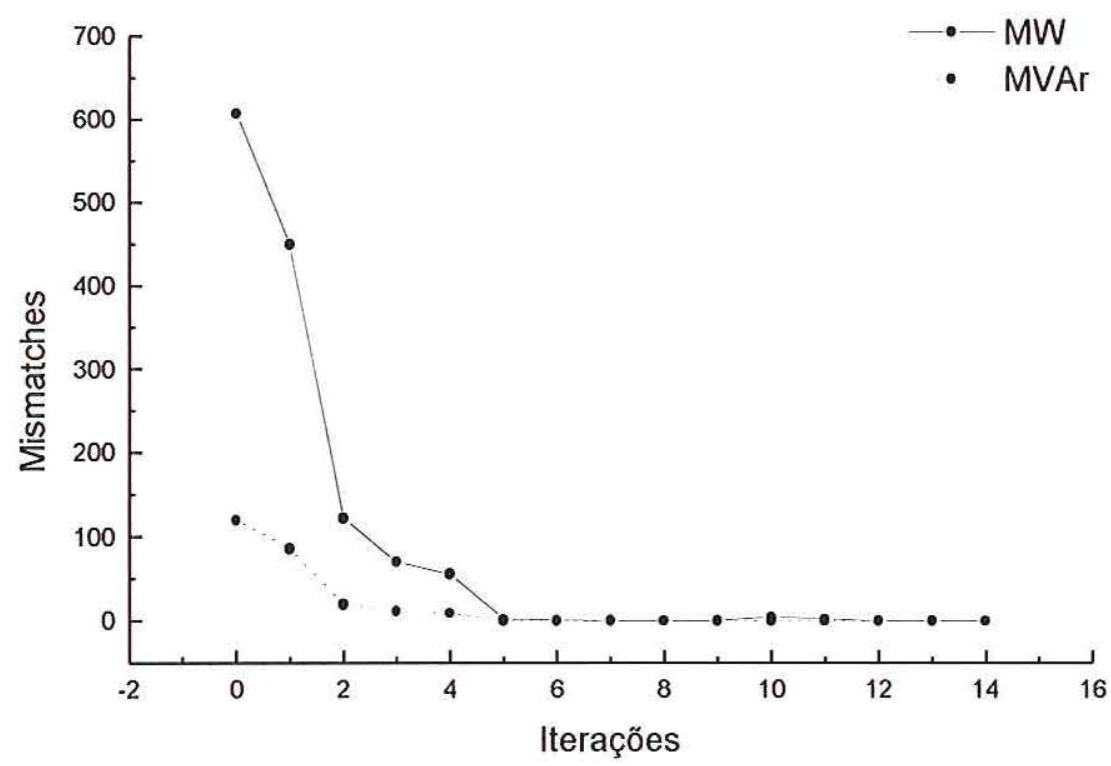

FIGURA 12 - As restrições $\Delta \mathrm{P}$ e $\Delta \mathrm{Q}$ a cada iteração

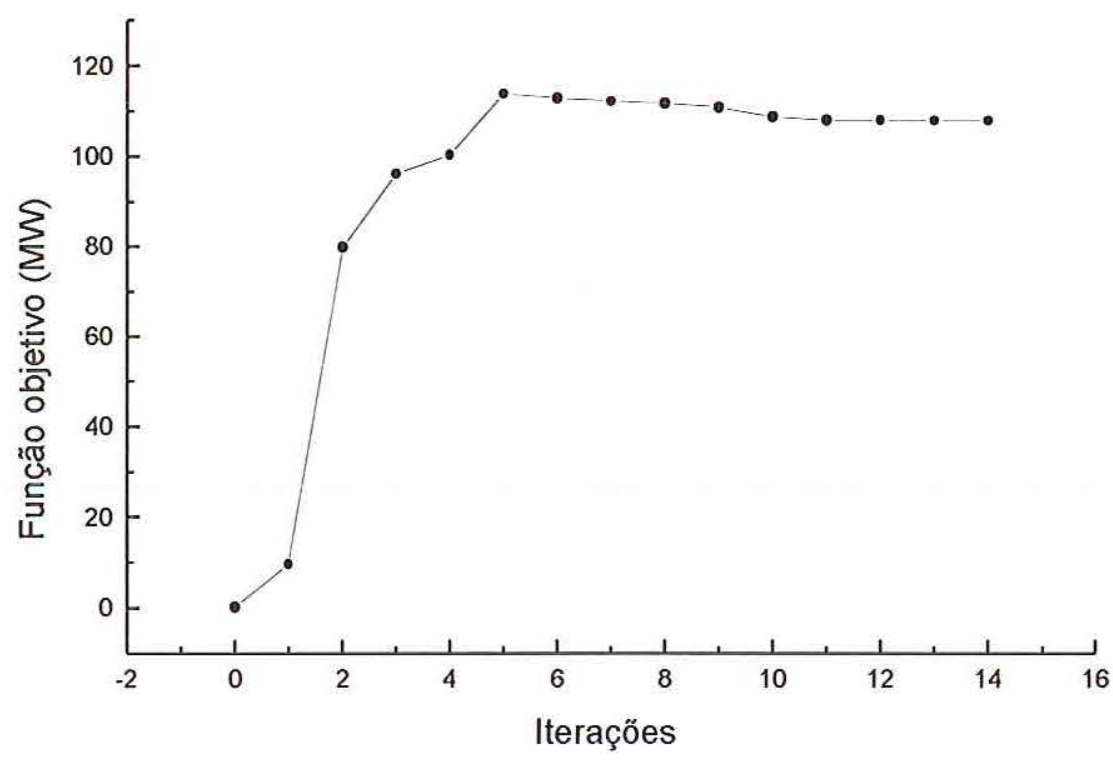

FIGURA 13 - Função objetivo 
Como pôde ser observado pelos gráficos anteriores, o processo convergiu em 14 iterações atingindo um valor final de função objetivo igual a 107,7298 MW. O tempo de CPU foi de 36,29 segundos e o vetor gradiente nulo, satisfazendo as condições de otimalidade.

No Apêndice 2 pode ser observado que as tensões nas barras 9, 30, 38, 66, 81 e 89 atingiram seus limites máximos, e desta forma teve-se que os multiplicadores $\pi_{3}<0$ e $\pi_{4}=0$, respectivos a estas barras, satisfizeram as condições de KKT.

A seguir será realizado uma análise entre o programa de fluxo de carga ótimo desenvolvido e o fluxo de carga convencional (FCC).

\section{6) ANÁLISE ENTRE O FLUXO DE CARGA ÓTIMO E O FLUXO DE CARGA CONVENCIONAL}

O programa de fluxo de carga convencional é utilizado por todas as concessionárias de energia elétrica, com o objetivo de conhecer o ponto de operação do sistema. Neste item o FCC será considerado como uma referência para o estudo do FCO, pois o mesmo fornece a solução de um sistema de equações sem otimizar nenhuma função objetivo. O programa de FCC utilizado foi o módulo de fluxo de carga do ANAREDE ${ }^{1}$.

As tabelas a seguir mostram que se obteve resultados diferentes quando se executou o FCO e o FCC para o mesmo o sistema, partindo do mesmo ponto inicial. Como pode ser observado, o FCO forneceu um ponto de operação melhor que o FCC, pois as perdas foram reduzidas e não houve violação de restrições.

${ }^{1}$ ANAREDE. Programa de fluxo de carga desenvolvido pelo CEPEL (Centro de Pesquisas de Energia Elétrica) em conjunto com a UNICAMP. 
a) Sistema de 3 barras:

TABELA 6 - Sistema de 3 barras (FCO x FCC)

\begin{tabular}{|c|c|c|c|c|}
\hline & iterações & Perdas (MW) & Injeção (MVAr) & restrições violadas \\
\hline FCO & 3 & 12,9153 & 129,4773 & 0 \\
\hline FCC & 4 & 17,7000 & 137,3000 & 1 \\
\hline
\end{tabular}

b) Sistema de 14 barras:

TABELA 7 - Sistema de 14 barras (FCO x FCC)

\begin{tabular}{|c|c|c|c|c|}
\hline & iterações & Perdas (MW) & Injeção (MVAr) & restrições violadas \\
\hline FCO & 3 & 12,4623 & 76,0142 & 0 \\
\hline FCC & 2 & 13,6000 & 82,6000 & 2 \\
\hline
\end{tabular}

c) Sistema de 30 barras:

TABELA 8 - Sistema de 30 barras (FCO x FCC)

\begin{tabular}{|c|c|c|c|c|}
\hline & iterações & Perdas (MW) & Injeção (MVAr) & restrições violadas \\
\hline FCO & 5 & 16,3241 & 147,7773 & 0 \\
\hline FCC & 2 & 17,6000 & 157,3000 & 4 \\
\hline
\end{tabular}

d) Sistema de 118 barras:

TABELA 9 - Sistema de 118 barras (FCO x FCC)

\begin{tabular}{|c|c|c|c|c|}
\hline & iterações & Perdas (MW) & Injeção (MVAr) & restrições violadas \\
\hline FCO & 16 & 107,6005 & 210,3282 & 0 \\
\hline FCC & 3 & 130,1000 & 618,5000 & 8 \\
\hline
\end{tabular}


Como pôde ser observado pelas tabelas $6,7,8$ e 9, o programa de fluxo de carga ótimo desenvolvido, forneceu um ponto de operação melhor, em relação ao fluxo de carga convencional, pois as perdas ativas do sistema foram em todos os casos menores. Nos três últimos casos o programa de FCO convergiu com um número maior de iterações, porém satisfez todas as restrições de operação do sistema, o que o programa de fluxo de carga convencional não conseguiu fazer.

Em todos os sistemas estudados, o programa de fluxo de carga ótimo forneceu uma quantidade menor de injeção de reativos em relação ao fluxo de carga convencional, melhorando desta forma o fator de potência do sistema. 


\section{CAPÍTULO 6}

\section{CONCLUSÕES}

A metodologia apresentada neste trabalho utiliza variáveis de folga para transformar as restrições de desigualdade em igualdades. As restrições do problema de otimização são acrescentadas na função objetivo através dos multiplicadores de Lagrange, e as variáveis de folga através da função barreira logarítmica. O método proposto deve ser inicializado a partir de pontos factíveis, para garantir a positividade das variáveis de folga, porém caso isto não aconteça o programa atribuirá um determinado valor positivo e diferente de zero para estas variáveis.

No capítulo anterior foram apresentados os resultados obtidos da aplicação do método primal-dual barreira-logarítmica para a solução do problema de fluxo de carga ótimo. Os resultados numéricos obtidos neste trabalho, mostram a eficiência do método quanto a rapidez de convergência e quanto aos valores obtidos na função objetivo. Este método procura satisfazer as restrições de desigualdade durante todo o processo iterativo, como foi mostrado no caso do sistema de 3 barras, cujas tensões lidas no banco de entrada de dados, estavam violando os seus limites inferiores, e ao fim da primeira iteração, todas as tensões já pertenciam a região factível.

O programa de fluxo de carga ótimo desenvolvido, apresentou resultados melhores em relação ao programa de fluxo de carga convencional, pois forneceu um ponto de operação que satisfez todas as restrições de operação do sistema elétrico de potência. Enquanto que o fluxo de carga convencional não conseguiu manter as restrições de operação dentro de seus limites preestabelecidos. 
Além do programa desenvolvido fornecer um ponto de operação factível para o sistema, este algoritmo apresenta como vantagem o fato das variáveis canalizadas do problema, como tensão, injeção de reativo e tap do transformador, não atingirem, na maioria das vezes, nenhum de seus limites, como pode ser observado na tabela 10 .

TABELA 10 - Restrições ativas na solução

\begin{tabular}{|c|c|c|c|c|c|c|}
\hline Sistema & $\mathrm{V}_{\max }$ & $\mathrm{V}_{\min }$ & $\mathrm{Q}_{\max }$ & $\mathrm{Q}_{\min }$ & $\operatorname{tap}_{\max }$ & $\operatorname{tap}_{\min }$ \\
\hline 14 barras & 0 & 0 & 0 & 0 & 0 & 0 \\
\hline 30 barras & 0 & 0 & 0 & 0 & 0 & 0 \\
\hline 118 barras & 6 & 0 & 0 & 0 & 0 & 0 \\
\hline
\end{tabular}

Esta característica do método fornece um ponto de operação, tal que o sistema possa operar dentro de seus limites de operação e não no limite, o que torna este algoritmo muito atrativo para as concessionárias de energia elétrica.

O fluxo de carga ótimo é um problema de programação não linear, e os métodos utilizados para resolvê-lo exigem que o usuário forneça algum valor para um determinado parâmetro, e no método primal-dual barreira-logarítmica implementado neste trabalho acontece o mesmo, o programa exige que o usuário forneça um valor para os parâmetros $\mu$ e $\beta$. Verificou-se que o algoritmo é muito sensível quanto à escolha destes parâmetros, podendo não satisfazer todas as restrições de operação do sistema ou até mesmo divergir para alguns valores.

Como o método tem como desvantagem a utilização de parâmetros empíricos, sugere-se para os futuros trabalhos realizados nesta área, a determinação do valor inicial do parâmetro de barreira por meios não empíricos, cancelando desta forma a dificuldade maior desta metodologia e fornecendo para o usuário um algoritmo que convirja sob qualquer condição. 


\section{BIBLIOGRAFIA}

BAZARAA, M.S.; JARVIS, J.J.; HANIF, D.S. (1990). Linear Programming and Network Flows. Second Edition, John Wiley \& Sons.

CARPENTIER, J.L. (1962). Contribution a L'etude du Dispatching Economique. Bull-Soc. Fr. Elec. Ser. B3, p. 431 - 447.

COSTA,G.R.M.; LANGONA, K.; ALVES, D. A. (1996). O Método de Newton Modificado Associado à Função Lagrangeana Aumentada Para a Resolução do Fluxo de Carga Ótimo. In: XI CONGRESSO BRASILEIRO DE AUTOMÁTICA, São Paulo, 1996. Anais. São Paulo, v. 1, p. 173-178.

DOMMEL, H.W.; TINNEY, W.F. (1968). Optimal Power Flows Solutions. IEEE Transactions on Power Apparatus and Systems, v. 87, p. 1866-1876, October.

FIACCO, A .V.; Mc CORMICK, G.P. (1968). Nonlinear Programming - Sequencial Unconstrained Optimization Techniques, John Wiley, New York.

FRERIS, L.L.; SASSON, A. M. (1968). Investigation of the Load Flow Problem. PROC IEEE, v.115, p.1459-1470.

FRISCH, K.R. (1955). The Logarithmic Potential Method of Convex Programming, Memorandum, University Institute of Economics, Oslo, Norway.

GILL, P.E.; MURRAY, W.; SAUNDERS, M.A (1986). On Projected Newton Barrier Methods for Linear Programming and an Equivalence to Karmarkar's Projective Method. Mathematical Programming, v. 36, p. 183 - 839. 
GRANVILLE, S. (1994) .Optimal Reactive Dispatch Through Interior Point Methods. IEEE Transactions on Power Systems, v. 9, p. 136-146, February.

\section{GUIA SIMPLIFICADO PARA USO DAS SUBROTINAS DO LABORATÓRIO} HARWELL NA SOLUÇÃO DE SISTEMAS LINEARES Ax = b.

Apostila do Departamento de Sistemas de Energia Elétrica - FEE / Unicamp Campinas - SP, Brasil

IRISARRI, G.D.; WANG, X.; TONG, J.; MOKHTARI, S. (1997). Maximum Loadability of Power Systems using Interior Point Non-Linear Optimization Method. IEEE Transactions on Power Systems, v. 12, p. 162-169, February.

KARMARKAR, N. (1984). A New Polynomial-Time Algorithm for Linear Programming. Combinatorica 4, v.4, p. 373 - 395.

LANGONA, K. (1994). O Método de Newton Modificado - Lagrangeana Aumentada para o Fluxo de Potência Ótimo. São Carlos. 87p. Dissertação (Mestrado) - Escola de Engenharia de São Carlos, Universidade de São Paulo.

MATUMOTO, L.T. (1996). Algoritmos de Pontos Interiores para Programação Linear e uma Extensão para Programação Linear por Partes. São Carlos. 56p. Dissertação (Mestrado) - Instituto de Ciências Matemáticas de São Carlos, Universidade de São Paulo.

MONTICELLI, A. (1983). Fluxo de Carga em Redes de Energia Elétrica. São Paulo, Edgard Blücher Ltda. Cap. 5, p. 75 - 100.

QUINTANA, V.H.; GOMEZ, A.; MARTINEZ, J.L. (1995). Nonlinear Optimal Power Flows by Logarithmic-Barrier Primal-Dual Algorithm. IEEE NAPS Meeting. 
RASHED, A.M.H.; KELLY, D.H. (1974). Optimal Load Flow Solutions Using Lagrangian Multipliers and the Hessian Matrix. IEEE Transactions on Power Apparatus and Systems, v. 93, p. 1292-1297, September/October.

SANTOS, A.; DECKMAN, S.; SOARES, S. (1988).A Dual Augmented Lagrangian Approach for Optimal Power Flow. IEEE Transactions on Power Apparatus and Systems, v. 3, p. 1020-1025, August.

SASSON, A. M.; VILORIA, F.; ABOYTES, F. (1973). Optimal Load Flow Solutions Using the Hessian Matrix .IEEE Transactions on Power Apparatus and Systems, v. 92, p. 31-41, January.

SHERKAT, V. R.; IKURA, Y. (1994). Experience with Interior Point Optimization Software for a Fuel Planning Application. IEEE Transactions on Power Systems, v. 9, p. 833-839, May.

SUN, D.I.; ASHLEY, B.; BREWER, B.; HUGLES, A.; TINNEY, W.F. (1984). Optimal Power Flow by Newton Approach. IEEE Transactions on Power Apparatus and Systems, v. 103, p. 2864-2875, October.

WU, Y.; DEBS, A.S.; MARSTEN, R.E. (1994). A Direct Nonlinear PredictorCorrector Primal-Dual Interior Point Algorithm for Optimal Power Flows. IEEE Transactions on Power Systems, v. 9, p. 876-883, May. 


\section{BANCO DE ENTRADA DE DADOS PARA O SISTEMA ELÉTRICO DE 3 BARRAS}

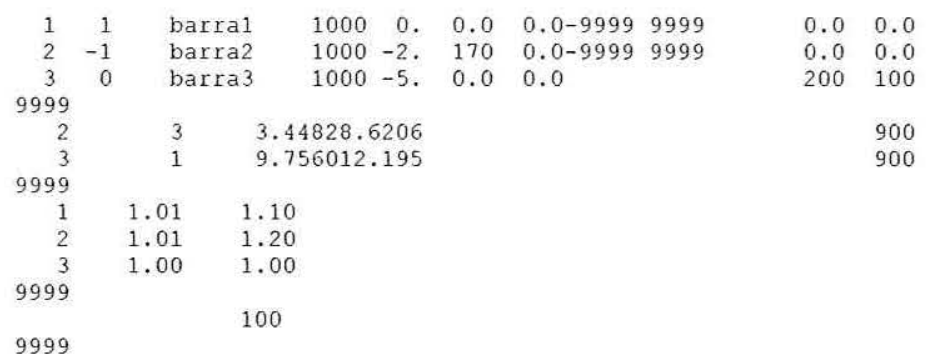

BANCO DE ENTRADA DE DADOS PARA O SISTEMA ELÉTRICO DE 14 BARRAS

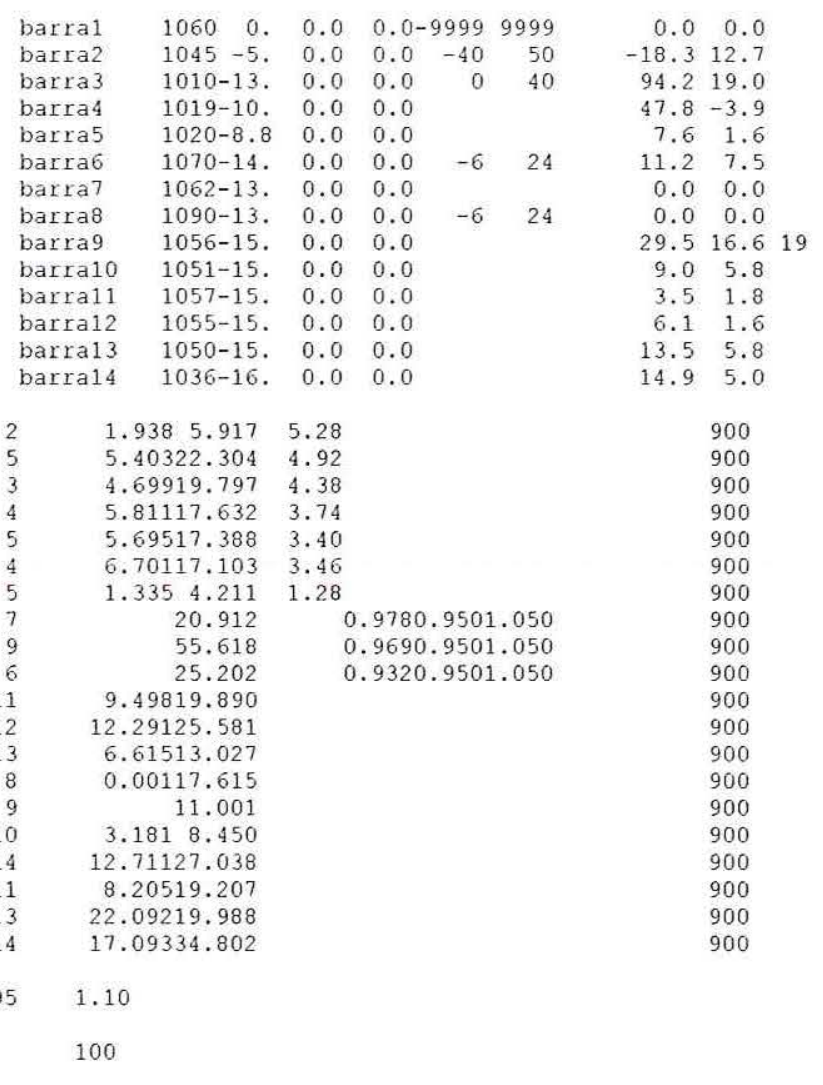


BANCO DE ENTRADA DE DADOS PARA O SISTEMA ELÉTRICO DE 30 BARRAS

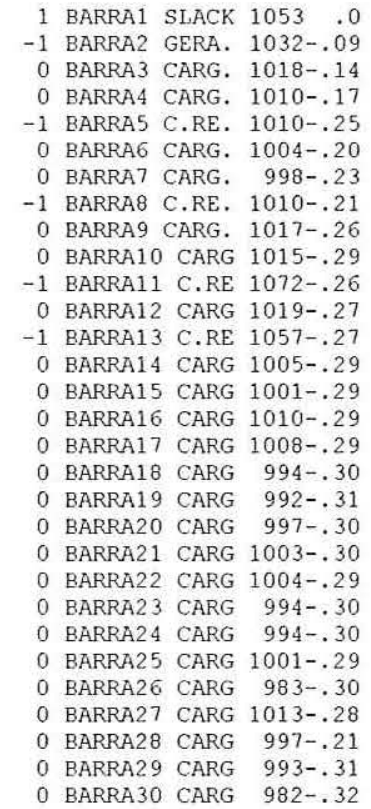

9999

0.9580 .9501 .050

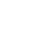




\section{BANCO DE ENTRADA DE DADOS PARA O SISTEMA ELÉTRICO DE 118 BARRAS}

\begin{tabular}{|c|c|c|c|c|}
\hline 12 & 1 & BARRA12 & 1000 & .0 \\
\hline 2 & 0 & BARRA2 & 1000 & .0 \\
\hline 3 & 0 & BARRA 3 & 1000 & .0 \\
\hline 4 & -1 & BARRA4 & 1000 & .0 \\
\hline 5 & 0 & BARRA5 & 1000 & .0 \\
\hline 6 & -1 & BARRA 6 & 1000 & .0 \\
\hline 7 & 0 & BARRA7 & 1000 & .0 \\
\hline 8 & -1 & BARRA8 & 1000 & .0 \\
\hline 9 & 0 & BARRA9 & 1000 & .0 \\
\hline 10 & -1 & BARRA10 & 1000 & .0 \\
\hline 11 & 0 & BARRA11 & 1000 & .0 \\
\hline 1 & -1 & BARRA1 & 1000 & .0 \\
\hline 13 & 0 & BARRA1 3 & 1000 & .0 \\
\hline 14 & 0 & BARRA14 & 1000 & .0 \\
\hline 15 & -1 & BARRA15 & 1000 & .0 \\
\hline 16 & 0 & BARRA16 & 1000 & .0 \\
\hline 17 & 0 & BARRA17 & 1000 & .0 \\
\hline 18 & -1 & BARRA18 & 1000 & .0 \\
\hline 19 & -1 & BARRA19 & 1000 & .0 \\
\hline 20 & 0 & BARRA20 & 1000 & .0 \\
\hline 21 & 0 & BARRA21 & 1000 & .0 \\
\hline 22 & 0 & BARRA22 & 1000 & .0 \\
\hline 23 & 0 & BARRA2 3 & 1000 & .0 \\
\hline 24 & 0 & BARRA2 4 & 1000 & .0 \\
\hline 25 & -1 & BARRA2 5 & 1000 & .0 \\
\hline 26 & -1 & BARRA2 6 & 1000 & .0 \\
\hline 27 & -1 & BARRA27 & 1000 & .0 \\
\hline 28 & 0 & BARRA28 & 1000 & .0 \\
\hline 29 & 0 & BARRA29 9 & 1000 & .0 \\
\hline 30 & 0 & BARRA 30 & 1000 & .0 \\
\hline 31 & -1 & BARRA31 & 1000 & .0 \\
\hline 32 & -1 & BARRA 32 & 1000 & .0 \\
\hline 33 & 0 & BARRA 33 & 1000 & .0 \\
\hline 34 & 0 & BARRA 34 & 1000 & .0 \\
\hline 35 & 0 & BARRA35 & 1000 & .0 \\
\hline 36 & -1 & BARRA 36 & 1000 & .0 \\
\hline 37 & 0 & BARRA37 & 1000 & .0 \\
\hline 38 & 0 & BARRA 38 & 1000 & .0 \\
\hline 39 & 0 & BARRA 39 & 1000 & .0 \\
\hline 40 & -1 & BARRA 40 & 1000 & .0 \\
\hline 41 & 0 & BARRA4 1 & 1000 & .0 \\
\hline 42 & -1 & BARRA4 2 & 1000 & .0 \\
\hline 43 & 0 & BARRA4 3 & 1000 & .0 \\
\hline 44 & 0 & BARRA44 & 1000 & .0 \\
\hline 45 & 0 & BARRA4 5 & 1000 & .0 \\
\hline 46 & -1 & BARRA4 6 & 1000 & .0 \\
\hline 47 & 0 & BARRA 47 & 1000 & .0 \\
\hline 48 & 0 & BARRA 48 & 1000 & .0 \\
\hline 49 & -1 & BARRA4 9 & 1000 & .0 \\
\hline 50 & 0 & BARRA 50 & 1000 & .0 \\
\hline 51 & 0 & BARRA 51 & 1000 & .0 \\
\hline 52 & 0 & BARRA 52 & 1000 & .0 \\
\hline 53 & 0 & BARRA 53 & 1000 & .0 \\
\hline 54 & -1 & BARRA54 & 1000 & .0 \\
\hline 55 & -1 & BARRA55 & 1000 & .0 \\
\hline 56 & -1 & BARRA56 6 & 1000 & .0 \\
\hline 57 & 0 & BARRA57 & 1000 & .0 \\
\hline 58 & 0 & BARRA 58 & 1000 & .0 \\
\hline 59 & -1 & BARRA 59 & 1000 & .0 \\
\hline 60 & 0 & BARRA 60 & 1000 & .0 \\
\hline 61 & -1 & BARRA 61 & 1000 & .0 \\
\hline 62 & -1 & BARRA 62 & 1000 & .0 \\
\hline 63 & 0 & BARRA 63 & 1000 & .0 \\
\hline 64 & 0 & BARRA 64 & 1000 & .0 \\
\hline 65 & -1 & BARRA 65 & 1000 & .0 \\
\hline 66 & -1 & BARRA 66 & 1000 & .0 \\
\hline 67 & 0 & BARRA 67 & 1000 & .0 \\
\hline 68 & 0 & BARRA68 & 1000 & .0 \\
\hline 69 & -1 & BARRA 69 & 1000 & .0 \\
\hline 70 & -1 & BARRA 70 & 1000 & .0 \\
\hline 71 & 0 & BARRA 71 & 1000 & .0 \\
\hline 72 & -1 & BARRA 72 & 1000 & .0 \\
\hline 73 & -1 & BARRA 73 & 1000 & .0 \\
\hline 74 & -1 & BARRA 74 & 1000 & .0 \\
\hline 75 & 0 & BARRA 75 & 1000 & .0 \\
\hline 76 & -1 & BARRA 76 & 1000 & .0 \\
\hline 77 & -1 & BARRA77 & 1000 & .0 \\
\hline 78 & 0 & BARRA78 & 1000 & .0 \\
\hline 79 & 0 & BARRA 79 & 1000 & .0 \\
\hline 80 & -1 & BARRA80 & 1000 & .0 \\
\hline
\end{tabular}

\begin{tabular}{|c|c|c|c|c|c|}
\hline $85 .-9999$ & 9999 & .0 & .0 & & 1 \\
\hline & & 20.0 & 9.0 & & 1 \\
\hline & & 39.0 & 10.0 & & 1 \\
\hline-50 & 50. & 39.0 & 12.0 & & 1 \\
\hline & & .0 & .0 & 40. & 1 \\
\hline-50. & 50. & 52.0 & 22.0 & & 1 \\
\hline & & 19.0 & 2.0 & & 1 \\
\hline-50 & 50. & 28.0 & 0.0 & & 1 \\
\hline & & .0 & .0 & & 1 \\
\hline-250 & 250. & -450 & . .0 & & 1 \\
\hline & & 70.0 & 23.0 & & \\
\hline-50 . & 50. & 51.0 & 27.0 & & 1 \\
\hline & & 34.0 & 16.0 & & 1 \\
\hline & & 14.0 & 1.0 & & 1 \\
\hline-50. & 50. & 90.0 & 30.0 & & 1 \\
\hline & & 25.0 & 10.0 & & 1 \\
\hline & & 11.0 & 3.0 & & 1 \\
\hline-50 & 50. & 60.0 & 34.0 & & 1 \\
\hline-50 & 115. & 45.0 & 25.0 & & 1 \\
\hline & & 18.0 & 3.0 & & 1 \\
\hline & & 14.0 & 8.0 & & 1 \\
\hline & & 10.0 & 5.0 & & 1 \\
\hline & & 7.0 & 3.0 & & 1 \\
\hline & & 13.0 & .0 & & 1 \\
\hline-250 & 250. & -220 & .0 & & 1 \\
\hline-250 . & 250. & -314 & .0 & & 1 \\
\hline-50 . & 50. & 71.0 & 13.0 & & 1 \\
\hline & & 17.0 & 7.0 & & 1 \\
\hline & & 24.0 & 4.0 & & 1 \\
\hline & & 0.0 & 0.0 & & 1 \\
\hline-50 & 50. & 36.0 & 27.0 & & 1 \\
\hline-50 . & 100. & 59.0 & 23.0 & & 1 \\
\hline & & 23.0 & 9.0 & & 1 \\
\hline & & 59.0 & 26.01 & 3.99 & 1 \\
\hline & & 33.0 & 9.0 & & 1 \\
\hline-50 . & 50. & 31.0 & 17.0 & & 1 \\
\hline & & .0 & .0 & 25. & 1 \\
\hline & & .0 & .0 & & 1 \\
\hline & & 27.0 & 11.0 & & 1 \\
\hline-50 . & 150. & 66.0 & 23.0 & & 1 \\
\hline & & 37.0 & 10.0 & & 1 \\
\hline-50. & 50. & 96.0 & 23.0 & & 1 \\
\hline & & 18.0 & 7.0 & & 1 \\
\hline & & 16.0 & 8.0 & 10 . & 1 \\
\hline & & 53.0 & 22.0 & 10. & 1 \\
\hline-50 & 50. & 9.0 & 10.0 & 10. & 1 \\
\hline & & 34.0 & .0 & & 1 \\
\hline & & 20.0 & 11.01 & 5.04 & 1 \\
\hline-250 . & 250. & -117 . & 30.0 & & 1 \\
\hline & & 17.0 & 4.0 & & 1 \\
\hline & & 17.0 & 8.0 & & 1 \\
\hline & & 18.0 & 5.0 & & 1 \\
\hline & & 23.0 & 11.0 & & 1 \\
\hline-50 & 150. & 65.0 & 32.0 & & 1 \\
\hline-50. & 150. & 63.0 & 22.0 & & 1 \\
\hline-50 . & 150. & 84.0 & 18.0 & & 1 \\
\hline & & 12.0 & 3.0 & & 1 \\
\hline & & 12.0 & 3.0 & & 1 \\
\hline-50. & 50. & 122.01 & 113.0 & & 1 \\
\hline & & 78.0 & 3.0 & & 1 \\
\hline-250 & 250. & -160 & .0 & & 1 \\
\hline-50 & 50. & 77.0 & 14.0 & & 1 \\
\hline & & .0 & .0 & & 1 \\
\hline & & .0 & .0 & & 1 \\
\hline-250 & 250. & -391 & .0 & & 1 \\
\hline & 250 . & -353 & 18.0 & & 1 \\
\hline & & 28.0 & 7.0 & & 1 \\
\hline & & .0 & .0 & & 1 \\
\hline-250 . & 250 & -329 & 0. & & 1 \\
\hline-50 & 50. & 66.0 & 20.0 & & 1 \\
\hline & & .0 & .0 & & 1 \\
\hline-50. & 50. & 12.0 & .0 & & 1 \\
\hline-50 & 50. & 6.0 & .0 & & 1 \\
\hline-50 & 80. & 68.0 & 27.0 & 12. & 1 \\
\hline & & 47.0 & 11.0 & & 1 \\
\hline-50 . & 100. & 68.0 & 36.0 & & 1 \\
\hline-50. & 50. & 61.0 & 28.0 & & 1 \\
\hline & & 71.0 & 26.0 & & 1 \\
\hline & & 39.0 & 32.0 & 20. & 1 \\
\hline-250. & 250. & -347 & 26.0 & & 1 \\
\hline
\end{tabular}




\begin{tabular}{|c|c|c|c|c|c|c|c|c|c|c|c|}
\hline 81 & 0 & BARRA81 & & 1000 & .0 & & & & & .0 & .0 \\
\hline 82 & 0 & BARRA82 & & 1000 & .0 & & & & & 54.0 & 27.0 \\
\hline 83 & 0 & BARRA8 3 & & 1000 & .0 & & & & & 20.0 & 10.0 \\
\hline 84 & 0 & BARRA84 & & 1000 & .0 & & & & & 11.0 & 7.0 \\
\hline 85 & -1 & BARRA 85 & & 1000 & .0 & & -50 & 80. & & 24.0 & 15.0 \\
\hline 86 & 0 & BARRA8 6 & & 1000 & .0 & & & & & 21.0 & 10.0 \\
\hline 87 & -1 & BARRA 87 & & 1000 & .0 & & -250 . & . 250 . & & -4.0 & .0 \\
\hline 88 & 0 & BARRA 88 & & 1000 & .0 & & & & & 48.0 & 10.0 \\
\hline 89 & -1 & BARRA6 9 & & 1000 & .0 & & -250 . & . 250 . & & -607 & .0 \\
\hline 90 & -1 & BARRA 90 & & 1000 & .0 & & -50 & . 100 . & & 163.0 & 42.0 \\
\hline 91 & -1 & BARRA91 & & 1000 & .0 & & -50 & . 50. & & 10.0 & .0 \\
\hline 92 & -1 & BARRA92 & & 1000 & .0 & & -50 & . 100 . & & 65.0 & 10.0 \\
\hline 93 & 0 & BARRA 93 & & 1000 & .0 & & & & & 12.0 & 7.0 \\
\hline 94 & 0 & BARRA94 & & 1000 & .0 & & & & & 30.0 & 16.0 \\
\hline 95 & 0 & BARRA95 & & 1000 & .0 & & & & & 42.0 & 31.0 \\
\hline 96 & 0 & BARRA96 & & 1000 & .0 & & & & & 38.0 & 15.0 \\
\hline 97 & 0 & BARRA97 & & 1000 & .0 & & & & & 15.0 & 9.0 \\
\hline 98 & 0 & BARRA98 & & 1000 & .0 & & & & & 34.0 & 8.0 \\
\hline 99 & -1 & BARRA99 & & 1000 & .0 & & -50 & 50. & & 42.0 & .0 \\
\hline 100 & -1 & BARRA 100 & & 1000 & .0 & & -250 . & . 250 . & & -215 & 18.0 \\
\hline 101 & 0 & BARRA 101 & & 1000 & .0 & & & & & 22.0 & 15.0 \\
\hline 102 & 0 & BARRA102 & & 1000 & .0 & & & & & 5.0 & 3.0 \\
\hline 103 & -1 & BARRA 103 & & 1000 & .0 & & -250 . & . 250 . & & -17.0 & 16.0 \\
\hline 104 & -1 & BARRA 104 & & 1000 & .0 & & -50 & 0. & & 38.0 & 25.0 \\
\hline 105 & -1 & BARRA 105 & & 1000 & .0 & & -50 . & 20. & & 31.0 & 26.0 \\
\hline 106 & 0 & BARRA106 & & 1000 & .0 & & & & & 43.0 & 16.0 \\
\hline 107 & -1 & BARRA 107 & & 1000 & .0 & & -50 & 60. & & 50.0 & 12.0 \\
\hline 108 & 0 & BARRA 108 & & 1000 & .0 & & & & & 2.0 & 1.0 \\
\hline 109 & 0 & BARRA 109 & & 1000 & .0 & & & & & 8.0 & 3.0 \\
\hline 110 & -1 & BARRA110 & & 1000 & .0 & & -50 & 0 . & & 39.0 & 30.0 \\
\hline 111 & -1 & BARRA111 & & 1000 & .0 & & -250 & 0. & & -36.0 & .0 \\
\hline 112 & -1 & BARRA 112 & & 1000 & .0 & & -50 & 50. & & 68.0 & 13.0 \\
\hline 113 & -1 & BARRA113 & & 1000 & .0 & & -50 & 0. & & 6.0 & .0 \\
\hline 114 & 0 & BARRA 114 & & 1000 & .0 & & & & & 8.0 & 3.0 \\
\hline 115 & 0 & BARRA115 & & 1000 & .0 & & & & & 22.0 & 7.0 \\
\hline 116 & -1 & BARRA116 & & 1000 & .0 & & -50 . & 0 . & & 0.0 & .0 \\
\hline 117 & 0 & BARRA 117 & & 1000 & .0 & & & & & 20.0 & 8.0 \\
\hline 118 & 0 & BARRA118 & & 1000 & .0 & & & & & 33.0 & 15.0 \\
\hline 9999 & & & & & & & & & & & \\
\hline 1 & & 2 & 3.03 & 9.99 & 2.540 & & & & 900. & 900. & \\
\hline 1 & & 3 & 1.29 & 4.24 & 1.082 & & & & 900. & 900. & \\
\hline 4 & & 5 & 0.176 & 60.798 & 0.210 & & & & 900. & 900. & \\
\hline 3 & & 5 & 2.41 & 10.80 & 2.840 & & & & 900. & 900. & \\
\hline 5 & & 6 & 1.19 & 5.40 & 1.426 & & & & 900. & 900. & \\
\hline 6 & & 7 & 0.459 & 92.08 & 0.550 & & & & 900. & 900. & \\
\hline 8 & & 9 & 0.244 & 43.05 & 116.62 & & & & 900. & 900. & \\
\hline 8 & & 5 & 0.00 & 2.67 & 0.000 & 1.0 & .95 & 1.05 & 900. & 900. & \\
\hline 9 & & 10 & 0.258 & 83.22 & 123.00 & & & & 900. & 900. & \\
\hline 4 & & 11 & 2.09 & 6.88 & 1.748 & & & & 900. & 900. & \\
\hline 5 & & 11 & 2.03 & 6.82 & 1.738 & & & & 900. & 900. & \\
\hline 11 & & 12 & 0.595 & 51.96 & 0.502 & & & & 900. & 900. & \\
\hline 2 & & 12 & 1.87 & 6.16 & 1.572 & & & & 900. & 900. & \\
\hline 3 & & 12 & 4.84 & 16.00 & 4.060 & & & & 900. & 900. & \\
\hline 7 & & 12 & 0.862 & 23.40 & 0.874 & & & & 900. & 900. & \\
\hline 11 & & 13 & 2.225 & 57.31 & 1.876 & & & & 900. & 900. & \\
\hline 12 & & 14 & 2.15 & 7.07 & 1.816 & & & & 900. & 900. & \\
\hline 13 & & 15 & 7.44 & 24.44 & 6.268 & & & & 900. & 900. & \\
\hline 14 & & 15 & 5.95 & 19.50 & 5.020 & & & & 900. & 900. & \\
\hline 12 & & 16 & 2.12 & 8.34 & 2.140 & & & & 900. & 900. & \\
\hline 15 & & 17 & 1.32 & 4.37 & 4.440 & & & & 900. & 900. & \\
\hline 16 & & 17 & 4.54 & 18.01 & 4.660 & & & & 900. & 900. & \\
\hline 17 & & 18 & 1.23 & 5.05 & 1.298 & & & & 900. & 900. & \\
\hline 18 & & 19 & 1.119 & 94.93 & 1.142 & & & & 900 . & 900. & \\
\hline 19 & & 20 & 2.52 & 11.70 & 2.980 & & & & 900. & 900. & \\
\hline 15 & & 19 & 1.20 & 3.94 & 1.010 & & & & 900. & 900. & \\
\hline 20 & & 21 & 1.83 & 8.49 & 2.160 & & & & 900. & 900. & \\
\hline 21 & & 22 & 2.09 & 9.70 & 2.460 & & & & 900. & 900. & \\
\hline 22 & & 23 & 3.42 & 15.90 & 4.040 & & & & 900. & 900. & \\
\hline 23 & & 24 & 1.35 & 4.92 & 4.980 & & & & 900. & 900. & \\
\hline 23 & & 25 & 1.56 & 8.00 & 8.640 & & & & 900. & 900. & \\
\hline 26 & & 25 & 0.00 & 3.82 & 0.000 & 1.0 & .95 & 1.05 & 900. & 900. & \\
\hline 25 & & 27 & 3.18 & 16.30 & 17.640 & & & & 900. & 900. & \\
\hline 27 & & 28 & 1.913 & 8.55 & 2.160 & & & & 900. & 900. & \\
\hline 28 & & 29 & 2.37 & 9.43 & 2.380 & & & & 900. & 900. & \\
\hline 30 & & 17 & 0.00 & 3.88 & 0.000 & 1.0 & .95 & 1.05 & 900. & 900. & \\
\hline 8 & & 30 & .431 & 5.04 & 51.400 & & & & 900. & 900. & \\
\hline 26 & & 30 & .799 & 8.60 & 91.800 & & & & 900. & 900. & \\
\hline 17 & & 31 & 4.74 & 15.63 & 3.990 & & & & 900. & 900. & \\
\hline 29 & & 31 & 1.08 & 3.31 & 0.830 & & & & 900. & 900. & \\
\hline 23 & & 32 & 3.17 & 11.53 & 11.730 & & & & 900. & 900. & \\
\hline 31 & & 32 & 2.98 & 9.85 & 2.510 & & & & 900. & 900. & \\
\hline 27 & & 32 & 2.29 & 7.55 & 1.926 & & & & 900. & 900. & \\
\hline 15 & & 33 & 3.80 & 12.44 & 3.194 & & & & 900. & 900. & \\
\hline 19 & & 34 & 7.52 & 24.70 & 6.320 & & & & 900. & 900. & \\
\hline 35 & & 36 & .224 & 1.02 & .268 & & & & 900. & 900. & \\
\hline 35 & & 37 & 1.10 & 4.97 & 1.318 & & & & 900. & 900. & \\
\hline 33 & & 37 & 4.15 & 14.20 & 3.660 & & & & 900. & 900. & \\
\hline
\end{tabular}




\begin{tabular}{|c|c|c|c|c|c|c|c|c|c|}
\hline 34 & 36 & .871 & 2.68 & 0.568 & & & & 900. & 900.0 \\
\hline 34 & 37 & .256 & 0.94 & 0.988 & & & & 900. & 900.0 \\
\hline 38 & 37 & 0.00 & 3.75 & 0.000 & 1.0 & .95 & 1.05 & 900. & 900.0 \\
\hline 37 & 39 & 3.21 & 10.60 & 2.700 & & & & 900. & 900.0 \\
\hline 37 & 40 & 5.93 & 16.80 & 4.200 & & & & 900. & 900.0 \\
\hline 30 & 38 & .464 & 5.40 & 42.200 & & & & 900. & 900.0 \\
\hline 39 & 40 & 1.84 & 6.05 & 1.552 & & & & 900 . & 900.0 \\
\hline 40 & 41 & 1.45 & 4.87 & 1.222 & & & & 900. & 900.0 \\
\hline 40 & 42 & 5.55 & 18.30 & 4.660 & & & & 900. & 900.0 \\
\hline 41 & 42 & 4.10 & 13.50 & 3.440 & & & & 900. & 900.0 \\
\hline 43 & 44 & 6.08 & 24.54 & 6.068 & & & & 900 . & 900.0 \\
\hline 34 & 43 & 4.13 & 16.81 & 4.226 & & & & 900. & 900.0 \\
\hline 44 & 45 & 2.24 & 9.01 & 2.240 & & & & 900. & 900.0 \\
\hline 45 & 46 & 4.00 & 13.56 & 3.320 & & & & 900. & 900.0 \\
\hline 46 & 47 & 3.80 & 12.70 & 3.160 & & & & 900. & 900.0 \\
\hline 46 & 48 & 6.01 & 18.90 & 4.720 & & & & 900. & 900.0 \\
\hline 47 & 49 & 1.91 & 6.25 & 1.604 & & & & 900. & 900.0 \\
\hline 42 & 49 & 7.15 & 32.30 & 8.600 & & & & 900. & 900.0 \\
\hline 42 & 49 & 7.15 & 32.30 & 8.600 & & & & 900 . & 900.0 \\
\hline 45 & 49 & 6.84 & 18.60 & 4.440 & & & & 900. & 900.0 \\
\hline 48 & 49 & 1.79 & 5.05 & 1.258 & & & & 900. & 900.0 \\
\hline 49 & 50 & 2.67 & 7.52 & 1.874 & & & & 900. & 900.0 \\
\hline 49 & 51 & 4.86 & 13.70 & 3.420 & & & & 900. & 900.0 \\
\hline 51 & 52 & 2.03 & 5.88 & 1.396 & & & & 900 . & 900.0 \\
\hline 52 & 53 & 4.05 & 16.35 & 4.058 & & & & 900. & 900.0 \\
\hline 53 & 54 & 2.63 & 12.20 & 3.110 & & & & 900. & 900.0 \\
\hline 49 & 54 & 7.30 & 28.90 & 7.380 & & & & 900. & 900.0 \\
\hline 49 & 54 & 8.69 & 29.10 & 7.310 & & & & 900. & 900.0 \\
\hline 54 & 55 & 1.69 & 7.07 & 2.020 & & & & 900. & 900.0 \\
\hline 54 & 56 & .275 & .955 & 0.732 & & & & 900. & 900.0 \\
\hline 55 & 56 & .488 & 1.51 & 0.374 & & & & 900. & 900.0 \\
\hline 56 & 57 & 3.43 & 9.66 & 2.420 & & & & 900. & 900.0 \\
\hline 50 & 57 & 4.74 & 13.40 & 3.320 & & & & 900 . & 900.0 \\
\hline 56 & 58 & 3.43 & 9.66 & 2.420 & & & & 900. & 900.0 \\
\hline 51 & 58 & 2.55 & 7.19 & 1.788 & & & & 900. & 900.0 \\
\hline 54 & 59 & 5.03 & 22.93 & 5.980 & & & & 900 . & 900.0 \\
\hline 56 & 59 & 8.25 & 25.10 & 5.690 & & & & 900. & 900.0 \\
\hline 56 & 59 & 8.03 & 23.90 & 5.360 & & & & 900. & 900.0 \\
\hline 55 & 59 & 4.739 & 21.58 & 5.646 & & & & 900 . & 900.0 \\
\hline 59 & 60 & 3.17 & 14.50 & 3.760 & & & & 900. & 900.0 \\
\hline 59 & 61 & 3.28 & 15.00 & 3.880 & & & & 900. & 900.0 \\
\hline 60 & 61 & .264 & 1.35 & 1.456 & & & & 900. & 900.0 \\
\hline 60 & 62 & 1.23 & 5.61 & 1.468 & & & & 900 . & 900.0 \\
\hline 61 & 62 & .824 & 3.76 & 0.980 & & & & 900 . & 900.0 \\
\hline 63 & 59 & 0.00 & 3.86 & 0.000 & 1.0 & .95 & 1.05 & 900. & 900.0 \\
\hline 63 & 64 & .172 & 2.00 & 21.60 & & & & 900. & 900.0 \\
\hline 64 & 61 & 0.00 & 2.68 & 0.000 & 1.0 & .95 & 1.05 & 900. & 900.0 \\
\hline 38 & 65 & .901 & 9.86 & 114.6 & & & & 900 . & 900.0 \\
\hline 64 & 65 & .269 & 3.02 & 38.00 & & & & 900. & 900.0 \\
\hline 49 & 66 & 1.80 & 9.19 & 2.480 & & & & 900. & 900.0 \\
\hline 49 & 66 & 1.80 & 9.19 & 2.480 & & & & 900. & 900.0 \\
\hline 62 & 66 & 4.82 & 21.80 & 5.780 & & & & 900 . & 900.0 \\
\hline 62 & 67 & 2.58 & 11.70 & 3.100 & & & & 900. & 900.0 \\
\hline 65 & 66 & 0.00 & 3.70 & 0.000 & 1.0 & .95 & 1.05 & 900. & 900.0 \\
\hline 66 & 67 & 2.24 & 10.15 & 2.682 & & & & 900. & 900.0 \\
\hline 65 & 68 & .138 & 1.60 & 63.80 & & & & 900. & 900.0 \\
\hline 47 & 69 & 8.44 & 27.78 & 7.092 & & & & 900. & 900.0 \\
\hline 49 & 69 & 9.85 & 32.40 & 8.280 & & & & 900 . & 900.0 \\
\hline 68 & 69 & 0.00 & 3.70 & 0.000 & 1.0 & .95 & 1.05 & 900. & 900.0 \\
\hline 69 & 70 & 3.00 & 12.70 & 12.20 & & & & 900 , & 900.0 \\
\hline 24 & 70 & 10.221 & 141.15 & 10.198 & & & & 900. & 900.0 \\
\hline 70 & 71 & .882 & 3.55 & 0.878 & & & & 900 . & 900.0 \\
\hline 24 & 72 & 4.88 & 19.60 & 4.880 & & & & 900. & 900.0 \\
\hline 71 & 72 & 4.46 & 18.00 & 4.444 & & & & 900. & 900.0 \\
\hline 71 & 73 & .866 & 4.54 & 1.178 & & & & 900. & 900.0 \\
\hline 70 & 74 & 4.01 & 13.23 & 3.368 & & & & 900. & 900.0 \\
\hline 70 & 75 & 4.28 & 14.10 & 3.600 & & & & 900. & 900.0 \\
\hline 69 & 75 & 4.05 & 12.20 & 12.40 & & & & 900. & 900.0 \\
\hline 74 & 75 & 1.23 & 4.06 & 1.034 & & & & 900 . & 900.0 \\
\hline 76 & 77 & 4.44 & 14.80 & 3.680 & & & & 900. & 900.0 \\
\hline 69 & 77 & 3.09 & 10.10 & 10.38 & & & & 900. & 900.0 \\
\hline 75 & 77 & 6.01 & 19.99 & 4.978 & & & & 900. & 900.0 \\
\hline 77 & 78 & .376 & 1.24 & 1.264 & & & & 900 . & 900.0 \\
\hline 78 & 79 & .546 & 2.44 & 0.648 & & & & 900. & 900.0 \\
\hline 77 & 80 & 1.70 & 4.85 & 4.720 & & & & 900. & 900.0 \\
\hline 77 & 80 & 2.94 & 10.50 & 2.280 & & & & 900. & 900.0 \\
\hline 79 & 80 & 1.56 & 7.04 & 1.870 & & & & 900 . & 900.0 \\
\hline 68 & 81 & .175 & 2.02 & 80.80 & & & & 900. & 900.0 \\
\hline 81 & 80 & 0.00 & 3.70 & 0.000 & 1.0 & .95 & 1.05 & 900 . & 900.0 \\
\hline 77 & 82 & 2.98 & 8.53 & 8.174 & & & & 900. & 900.0 \\
\hline 82 & 83 & 1.12 & 3.665 & 3.796 & & & & 900 . & 900.0 \\
\hline 83 & 84 & 6.25 & 13.20 & 2.580 & & & & 900. & 900.0 \\
\hline 83 & 85 & 4.30 & 14.80 & 3.480 & & & & 900 . & 900.0 \\
\hline 84 & 85 & 3.02 & 6.41 & 1.234 & & & & 900. & 900.0 \\
\hline 85 & 86 & 3.50 & 12.30 & 2.760 & & & & 900 . & 900.0 \\
\hline 86 & 87 & 2.828 & 20.74 & 4.450 & & & & 900. & 900.0 \\
\hline 85 & 88 & 2.00 & 10.20 & 2.760 & & & & 900. & 900.0 \\
\hline
\end{tabular}




\begin{tabular}{|c|c|c|c|c|c|c|}
\hline 85 & 89 & 2.39 & 17.30 & 4.700 & 900. & 900.0 \\
\hline 88 & 89 & 1.39 & 7.12 & 1.934 & 900. & 900.0 \\
\hline 89 & 90 & 5.18 & 18.80 & 5.280 & 900. & 900.0 \\
\hline 89 & 90 & 2.38 & 9.97 & 10.60 & 900. & 900.0 \\
\hline 90 & 91 & 2.54 & 8.36 & 2.140 & 900. & 900.0 \\
\hline 89 & 92 & 0.99 & 5.05 & 5.480 & 900. & 900.0 \\
\hline 89 & 92 & 3.93 & 15.81 & 4.140 & 900. & 900.0 \\
\hline 91 & 92 & 3.87 & 12.72 & 3.268 & 900. & 900.0 \\
\hline 92 & 93 & 2.58 & 8.48 & 2.180 & 900. & 900.0 \\
\hline 92 & 94 & 4.81 & 15.80 & 4.060 & 900. & 900.0 \\
\hline 93 & 94 & 2.23 & 7.32 & 1.876 & 900. & 900.0 \\
\hline 94 & 95 & 1.32 & 4.34 & 1.110 & 900. & 900.0 \\
\hline 80 & 96 & 3.56 & 18.20 & 4.940 & 900 . & 900.0 \\
\hline 82 & 96 & 1.62 & 5.30 & 5.440 & 900. & 900.0 \\
\hline 94 & 96 & 2.69 & 8.69 & 2.300 & 900. & 900.0 \\
\hline 80 & 97 & 1.83 & 9.34 & 2.540 & 900. & 900.0 \\
\hline 80 & 98 & 2.38 & 10.80 & 2.860 & 900. & 900.0 \\
\hline 80 & 99 & 4.54 & 20.60 & 5.460 & 900. & 900.0 \\
\hline 92 & 100 & 6.48 & 29.50 & 7.720 & 900. & 900.0 \\
\hline 94 & 100 & 1.78 & 5.80 & 6.040 & 900. & 900.0 \\
\hline 95 & 96 & 1.71 & 5.47 & 1.474 & 900. & 900.0 \\
\hline 96 & 97 & 1.73 & 8.85 & 2.400 & 900. & 900.0 \\
\hline 98 & 100 & 3.97 & 17.90 & 4.760 & 900. & 900.0 \\
\hline 99 & 100 & 1.80 & 8.13 & 2.160 & 900. & 900.0 \\
\hline 100 & 101 & 2.77 & 12.62 & 3.280 & 900. & 900.0 \\
\hline 92 & 102 & 1.23 & 5.59 & 1.464 & 900. & 900.0 \\
\hline 101 & 102 & 2.46 & 11.20 & 2.840 & 900. & 900.0 \\
\hline 100 & 103 & 1.60 & 5.25 & 5.360 & 900. & 900.0 \\
\hline 100 & 104 & 4.51 & 20.40 & 5.410 & 900. & 900.0 \\
\hline 103 & 104 & 4.66 & 15.84 & 4.070 & 900. & 900.0 \\
\hline 103 & 105 & 5.35 & 16.25 & 4.080 & 900. & 900.0 \\
\hline 100 & 106 & 6.05 & 22.90 & 6.200 & 900. & 900.0 \\
\hline 104 & 105 & .994 & 3.78 & 0.986 & 900. & 900.0 \\
\hline 105 & 106 & 1.40 & 5.47 & 1.434 & 900. & 900.0 \\
\hline 105 & 107 & 5.30 & 18.30 & 4.720 & 900. & 900.0 \\
\hline 105 & 108 & 2.61 & 7.03 & 1.844 & 900. & 900.0 \\
\hline 106 & 107 & 5.30 & 18.30 & 4.720 & 900. & 900.0 \\
\hline 108 & 109 & 1.05 & 2.88 & 0.760 & 900. & 900.0 \\
\hline 103 & 110 & 3.906 & 618.13 & 4.610 & 900. & 900.0 \\
\hline 109 & 110 & 2.78 & 7.62 & 2.020 & 900. & 900.0 \\
\hline 110 & 111 & 2.20 & 7.55 & 2.000 & 900. & 900.0 \\
\hline 110 & 112 & 2.47 & 6.40 & 6.200 & 900. & 900.0 \\
\hline 17 & 113 & .913 & 3.01 & 0.768 & 900. & 900.0 \\
\hline 32 & 113 & 6.15 & 20.30 & 5.180 & 900. & 900.0 \\
\hline 32 & 114 & 1.35 & 6.12 & 1.628 & 900. & 900.0 \\
\hline 27 & 115 & 1.64 & 7.41 & 1.972 & 900 . & 900.0 \\
\hline 114 & 115 & 0.23 & 1.04 & 0.276 & 900. & 900.0 \\
\hline 68 & 116 & .034 & .405 & 16.40 & 900. & 900.0 \\
\hline 12 & 117 & 3.29 & 14.00 & 3.580 & 900. & 900.0 \\
\hline 75 & 118 & 1.45 & 4.81 & 1.198 & 900. & 900.0 \\
\hline 76 & 118 & 1.64 & 5.44 & 1.356 & 900. & 900.0 \\
\hline \multicolumn{7}{|l|}{9999} \\
\hline & 0.95 & \multicolumn{5}{|l|}{1.1} \\
\hline & & \multirow{2}{*}{\multicolumn{5}{|c|}{100}} \\
\hline & & & & & & \\
\hline
\end{tabular}




\section{APÊNDICE 2}

\section{BANCO DE SAIDA DE DADOS PARA O SISTEMA ELÉTRICO DE 3 BARRAS}

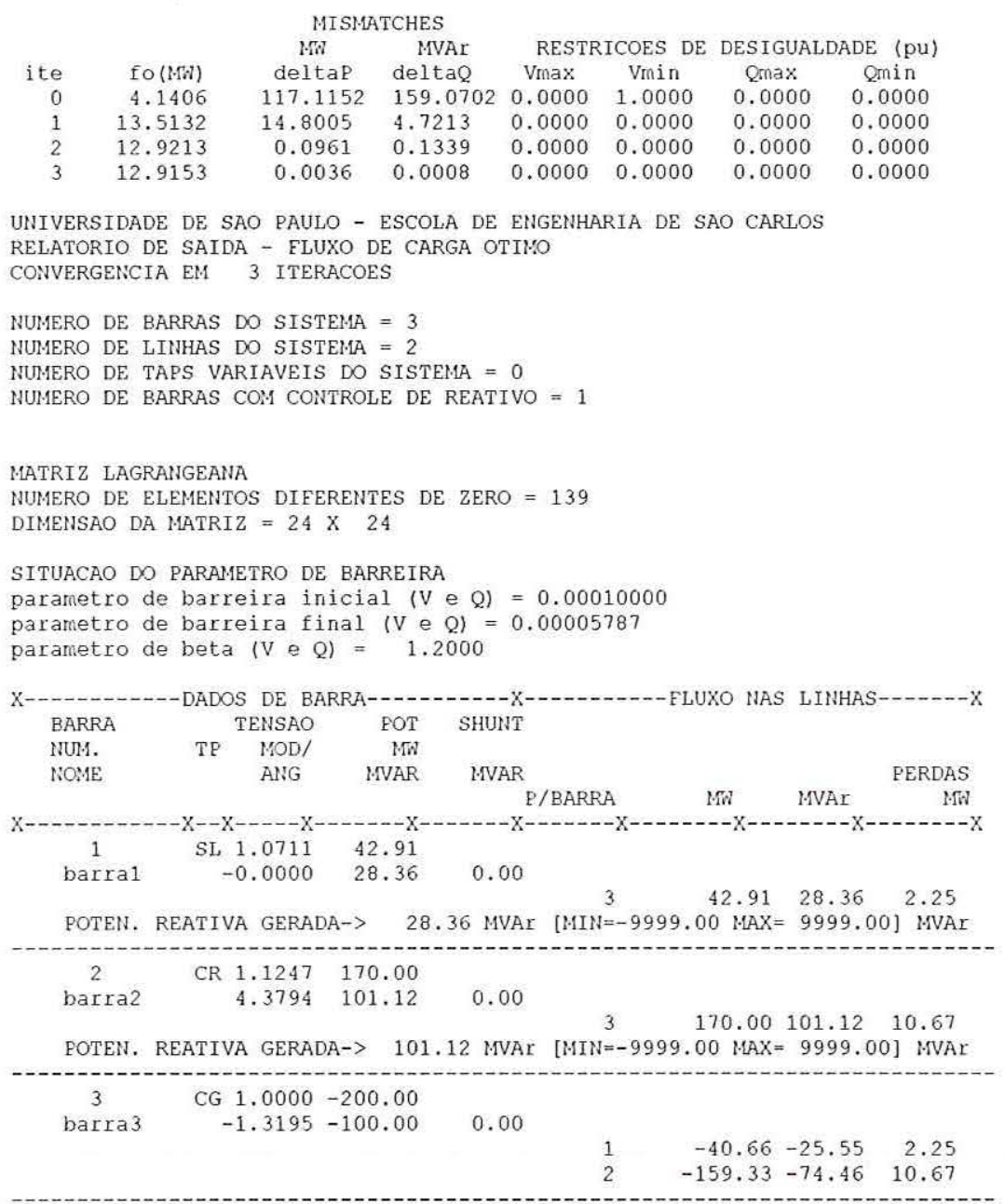

PERDAS ATIVAS DO SISTEMA $=12.9153 \mathrm{MW}$

TOTAL DE INJECAO DE REATIVO DO SISTEMA $=129.4773 \mathrm{MVAr}$

\begin{tabular}{ccccc} 
SUMARIO DOS MULTIPLICADORES E VARIAVEIS DE FOLGA DAS TENSOES \\
barra & 53 & 54 & pi 3 & pi \\
1 & 0.0289 & 0.0611 & -0.0024 & 0.0011 \\
2 & 0.0753 & 0.1147 & -0.0009 & 0.0006 \\
\hline
\end{tabular}




\section{BANCO DE SAIDA DE DADOS PARA O SISTEMA ELÉTRICO DE 14 BARRAS}

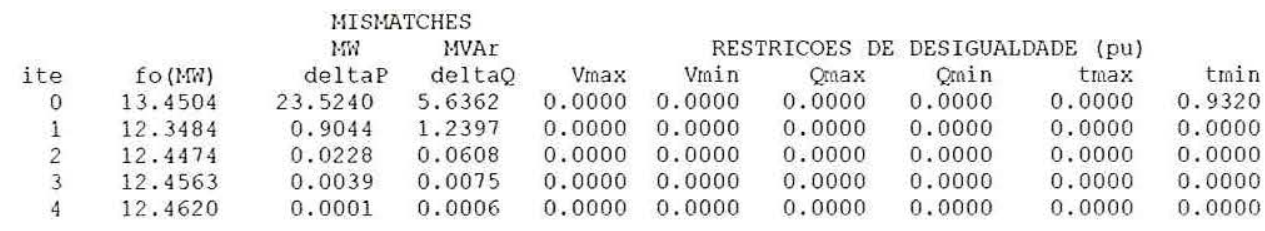

UNIVERSIDADE DE SAO PAULO - ESCOLA DE ENGENHARIA DE SAO CARLOS RELATORIO DE SAIDA - FLUXO DE CARGA OTI\%O CONVERGEICIA EM 4 ITERACOES

NUMERO DE BARRAS DO SISTEM.MA $=14$

NUMERO DE LINHAS DO SISTEMA $=20$

NUMERO DE TAPS VARIAVEIS DO SISTEMAA = 3

NUMERO DE BARRAS COM CONTROLE DE REATIVO $=4$

MATRIZ LAGRANGEANA

NUMERO DE ELEMENTOS DIFERENTES DE ZERO $=1023$

DIMENSAO DA MATRIZ $=136 \times 136$

SITUACAO DO PARAMETRO DE BARREIRA

parametro de barreira inicial (V e Q) $=0.00100000$

parametro de barreira final $(\mathrm{V}$ e $Q)=0.00096098$

parametro de beta $(\mathrm{V}$ e Q $)=1.0100$

parametro de barreira inicial (tap) $=0.01000000$

parametro de barreira final (tap) $=0.00960980$

parametro de beta $($ tap $)=1.0100$

\begin{tabular}{|c|c|c|c|c|c|c|c|}
\hline BARRA & TENSAO & FOT & SHUNT & & & & \\
\hline NUM. & TP $\quad \mathrm{KOD} /$ & $\mathrm{Mm}$ & & & & & \\
\hline NOME & ANG & MVAR & MVAR & & & & PERDAS \\
\hline & & & & BARRA & $\mathrm{Mn}$ & MVAr & $\mathrm{MW}$ \\
\hline 1 & $X--X-----X-$ & $-1-x--$ & $-\cdots-x-1$ & $-\infty-1$ & $-x-x-1$ & $-\cdots-x-\cdots$ & ל \\
\hline 1 & SL 1.0998 & 231.46 & & & & & \\
\hline barral & -0.0000 & 2.35 & 0.00 & & & & \\
\hline & & & & 2 & 156.21 & -4.48 & 3.91 \\
\hline & & & & 5 & 75.25 & 6.83 & 2.57 \\
\hline POTEN. & ATIVA GERAD & 2.35 & 5 MVAr & {$[\mathrm{MIN}]=$} & $999.00 \mathrm{MAX}=$ & $9999.00]$ & )] MVAr \\
\hline 2 & CR 1.0763 & 18.30 & & & & & \\
\hline barra 2 & -4.4903 & 12.11 & 0.00 & & & & \\
\hline & & & & 1 & -152.30 & 10.17 & 3.91 \\
\hline & & & & 3 & 73.25 & 2.84 & 2.19 \\
\hline & & & & 4 & 56.05 & -0.86 & 1.58 \\
\hline & & & & 5 & 41.29 & -0.04 & 0.84 \\
\hline FOTEN. & ATIVA GERAD & 24.81 & 1 MVAr & {$[\mathrm{MIN}]=$} & -40.00 MAX $=$ & $50.00]$ & ) MVAr \\
\hline 3 & CR 1.0429 & -94.20 & & & & & \\
\hline barra3 & -11.7835 & 6.10 & 0.00 & & & & \\
\hline & & & & 2 & -71.07 & 1.46 & 2.19 \\
\hline & & & & 4 & -23.13 & 4.64 & 0.36 \\
\hline POTEN. & ATIVA GERAD & 25.10 & 0 HVAr & {$[M I N=$} & $0.00 \mathrm{MAX}=$ & $40.00]$ & ] MVAr \\
\hline 4 & CG 1.0479 & -47.80 & & & & & \\
\hline barra 4 & -9.4791 & 3.90 & 0.00 & & & & \\
\hline & & & & 2 & -54.48 & 1.43 & 1.58 \\
\hline & & & & 3 & 23.49 & -7.52 & 0.36 \\
\hline & & & & 5 & -62.50 & 6.19 & 0.48 \\
\hline & & & & 7 & 29.08 & 1.13 & 0.00 \\
\hline & & & & 9 & 16.61 & 2.67 & 0.00 \\
\hline 5 & CG 1.0534 & -7.60 & & & & & \\
\hline barra5 & -8.0652 & -1.60 & 0.00 & & & & \\
\hline & & & & 1 & -72.68 & -1.92 & 2.57 \\
\hline & & & & 2 & -40.45 & -1.25 & 0.84 \\
\hline & & & & 4 & 62.98 & -6.08 & 0.48 \\
\hline & & & & 6 & 42.55 & 7.66 & 0.00 \\
\hline 6 & CR 1.0426 & -11.20 & & & & & \\
\hline barra6 & -13.6555 & 7.30 & 0.00 & & & & \\
\hline & & & & 5 & -42.55 & -3.43 & 0.00 \\
\hline & & & & 11 & 6.46 & 1.99 & 0.04 \\
\hline & & & & 12 & 7.63 & 2.33 & 0.07 \\
\hline
\end{tabular}




\begin{tabular}{|c|c|c|c|c|c|c|c|}
\hline POTEN. & ATIVA GERAL & $1->$ & 30 MVAr & $\begin{array}{r}13 \\
{[\mathrm{MIN}=}\end{array}$ & $\begin{array}{c}17.27 \\
-6.00 \mathrm{MAX}=\end{array}$ & $\begin{array}{l}6.42 \\
24.00]\end{array}$ & $\begin{array}{l}0.21 \\
\text { MVAr }\end{array}$ \\
\hline $\begin{array}{c}7 \\
\text { barra? }\end{array}$ & $\begin{array}{r}\text { CG } 1.0454 \\
-12.6667\end{array}$ & $\begin{array}{r}-0.00 \\
0.00\end{array}$ & 0.00 & $\begin{array}{l}4 \\
8 \\
9\end{array}$ & $\begin{array}{r}-29.08 \\
0.00 \\
29.08\end{array}$ & $\begin{array}{r}0.48 \\
-8.82 \\
8.34\end{array}$ & $\begin{array}{l}0.00 \\
0.00 \\
0.00\end{array}$ \\
\hline $\begin{array}{c}8 \\
\text { barra8 } \\
\text { POTEN. }\end{array}$ & $\begin{array}{r}\text { CR } 1.0602 \\
-12.6667 \\
\text { ATIVA GERAD }\end{array}$ & $\begin{array}{r}0.00 \\
8.95 \\
->\quad 8\end{array}$ & $\begin{array}{l}0.00 \\
95 \text { MVAr }\end{array}$ & $\begin{array}{r}7 \\
{\left[\mathrm{MIN}^{7}=\right.}\end{array}$ & $\begin{array}{c}0.00 \\
-6.00 \text { ‥ }\end{array}$ & $\begin{array}{l}8.95 \\
24.00]\end{array}$ & $\begin{array}{l}0.00 \\
\text { MVAr }\end{array}$ \\
\hline $\begin{array}{c}9 \\
\text { barra9 }\end{array}$ & $\begin{array}{r}\text { CG } 1.0370 \\
-14.3574\end{array}$ & $\begin{array}{l}-29.50 \\
-16.60\end{array}$ & 18.32 & $\begin{array}{r}4 \\
7 \\
10 \\
14\end{array}$ & $\begin{array}{r}-16.61 \\
-29.08 \\
6.11 \\
10.08\end{array}$ & $\begin{array}{r}-1.23 \\
-7.42 \\
5.76 \\
4.61\end{array}$ & $\begin{array}{l}0.00 \\
0.00 \\
0.02 \\
0.15\end{array}$ \\
\hline $\begin{array}{l}10 \\
\text { barra10 }\end{array}$ & $\begin{array}{r}\text { CG } 1.0305 \\
-14.5358\end{array}$ & $\begin{array}{l}-9.00 \\
-5.80\end{array}$ & 0.00 & $\begin{array}{r}9 \\
11\end{array}$ & $\begin{array}{l}-6.09 \\
-2.91\end{array}$ & $\begin{array}{l}-5.71 \\
-0.09\end{array}$ & $\begin{array}{l}0.02 \\
0.01\end{array}$ \\
\hline$\stackrel{11}{\text { barra11 }}$ & $\begin{array}{r}\text { CG } 1.0330 \\
-14.2385\end{array}$ & $\begin{array}{l}-3.50 \\
-1.80\end{array}$ & 0.00 & $\begin{array}{r}6 \\
10\end{array}$ & $\begin{array}{r}-6.42 \\
2.92\end{array}$ & $\begin{array}{r}-1.91 \\
0.11\end{array}$ & $\begin{array}{l}0.04 \\
0.01\end{array}$ \\
\hline $\begin{array}{l}12 \\
\text { barra12 }\end{array}$ & $\begin{array}{r}\text { CG } 1.0280 \\
-14.5458\end{array}$ & $\begin{array}{l}-6.10 \\
-1.60\end{array}$ & 0.00 & $\begin{array}{r}6 \\
13\end{array}$ & $\begin{array}{r}-7.56 \\
1.46\end{array}$ & $\begin{array}{r}-2.18 \\
0.58\end{array}$ & $\begin{array}{l}0.07 \\
0.01\end{array}$ \\
\hline $\begin{array}{l}13 \\
\text { barra13 }\end{array}$ & $\begin{array}{r}\text { CG } 1.0238 \\
-14.6349\end{array}$ & $\begin{array}{r}-13.50 \\
-5.80\end{array}$ & 0.00 & $\begin{array}{r}6 \\
12 \\
14\end{array}$ & $\begin{array}{r}-17.06 \\
-1.45 \\
5.01\end{array}$ & $\begin{array}{r}-6.01 \\
-0.57 \\
0.78\end{array}$ & $\begin{array}{l}0.21 \\
0.01 \\
0.04\end{array}$ \\
\hline $\begin{array}{l}14 \\
\text { barra14 }\end{array}$ & $\begin{array}{r}\text { CG } 1.0129 \\
-15.5242\end{array}$ & $\begin{array}{r}-14.90 \\
-5.00\end{array}$ & 0.00 & $\begin{array}{r}9 \\
13\end{array}$ & $\begin{array}{l}-9.93 \\
-4.97\end{array}$ & $\begin{array}{l}-4.30 \\
-0.70\end{array}$ & $\begin{array}{l}0.15 \\
0.04\end{array}$ \\
\hline
\end{tabular}

PERDAS ATIVAS DO SISTEMA $=12.4620 \mathrm{~mW}$

TOTAL DE INJECAO DE REATIVO DO SISTEMA = 76.0142 MVAR

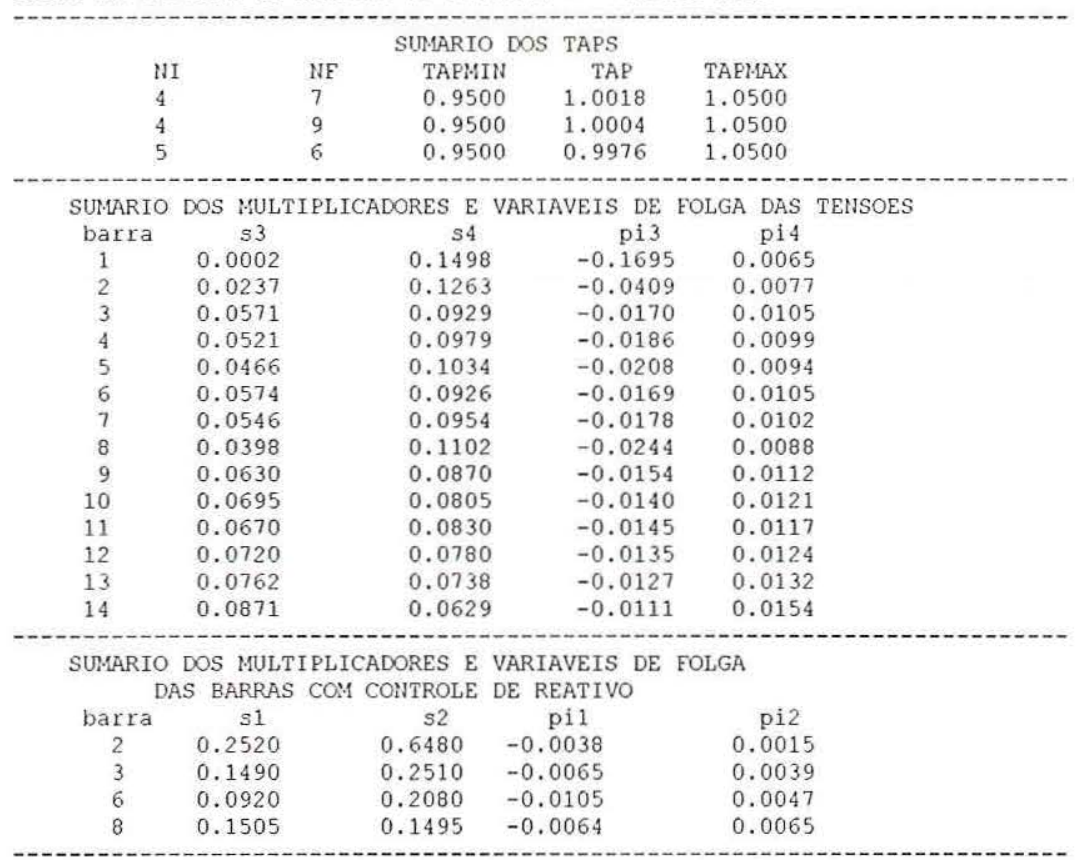




\section{BANCO DE SAIDA DE DADOS PARA O SISTEMA ELÉTRICO DE 30 BARRAS}

\begin{tabular}{rrrrrrrrrr}
\multicolumn{1}{c}{ MISMATCHES } \\
ite & fo(Mw) & deltaP & MVAr & deltaQ & Vmax & \multicolumn{2}{c}{ RESTRICOES DE DESIGUALDADE (pu) } \\
0 & 1.4427 & 93.6054 & 41.9110 & 0.0000 & 0.0000 & 0.2869 & 0.0000 & 0.0000 & 0.0000 \\
1 & 2.7017 & 70.3972 & 30.9842 & 0.0000 & 0.0000 & 0.1339 & 0.0000 & 0.0000 & 0.0000 \\
2 & 6.9231 & 36.5924 & 15.3249 & 0.0000 & 0.0000 & 0.4030 & 0.0000 & 0.0000 & 0.0000 \\
3 & 16.0991 & 1.2913 & 1.0961 & 0.0000 & 0.0000 & 0.3987 & 0.0000 & 0.0000 & 0.0000 \\
4 & 16.3508 & 0.0071 & 0.0200 & 0.0000 & 0.0000 & 0.0000 & 0.0000 & 0.0000 & 0.0000 \\
5 & 16.3564 & 0.0004 & 0.0001 & 0.0000 & 0.0000 & 0.0000 & 0.0000 & 0.0000 & 0.0000
\end{tabular}

UNIVERSIDADE DE SAO PAULO - ESCOLA DE ENGENHARIA DE SAO CARLOS RELATORIO DE SAIDA - FLUXO DE CARGA OTIYO CONVERGENCIA EM 5 ITERACOES

NUMERO DE BARRAS DO SISTEMA $=30$

NUAERO DE LINHAS DO SISTEMA $=41$

NUMERO DE TAPS VARIAVEIS DO SISTEMA $=4$

NUMERO DE BARRAS COM CONTROLE DE REATIVO $=5$

MATRIZ LAGRANGEANA

NUMERO DE ELEMENTOS DIFERENTES DE ZERO $=1909$

DIMENSAO DA MATRIZ $=272 \times 272$

SITUACAO DO PARAMETRO DE BARREIRA

parametro de barreira inicial (V e Q) $=0.00100000$

parametro de barreira final $(V \in Q)=0.00040188$

parametro de beta $(V$ e $Q)=1.2000$

parametro de barreira inicial (tap) $=1.00000000$

parametro de barreira final (tap) $=0.62092132$

parametro de beta $($ tap $)=1.1000$

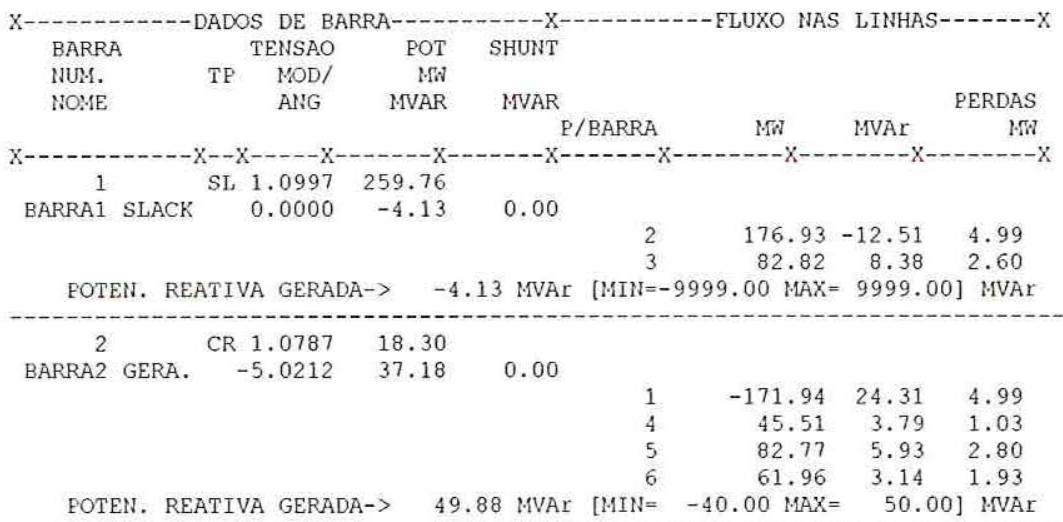

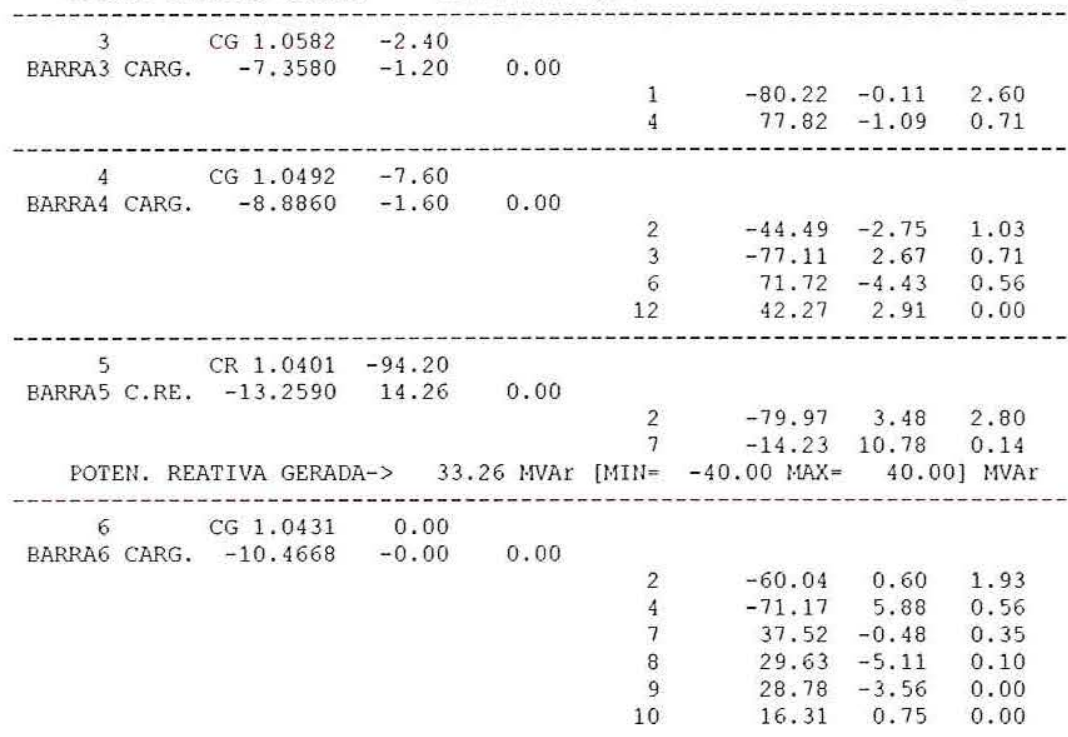




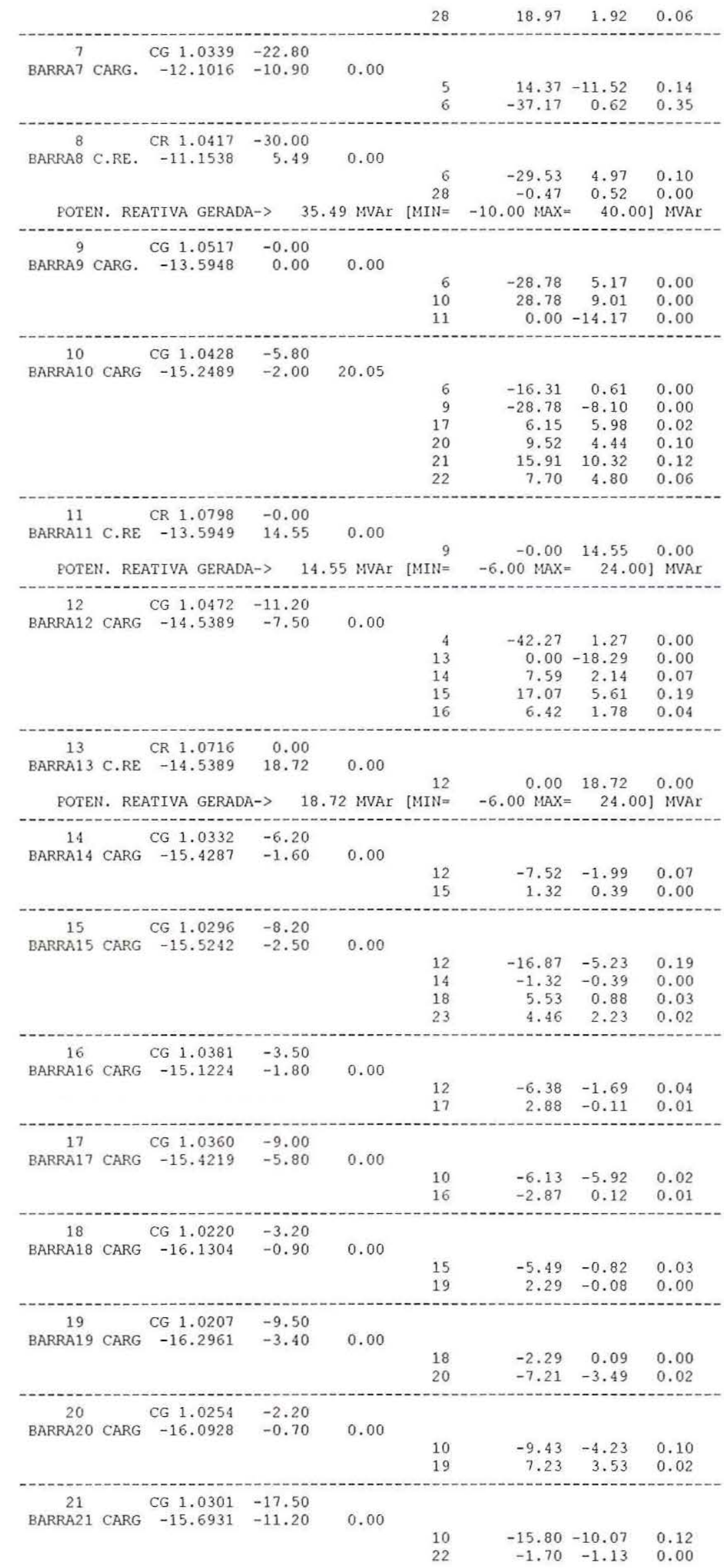




\begin{tabular}{|c|c|c|c|c|c|c|c|c|}
\hline $\begin{array}{c}22 \\
\text { BARRA22 }\end{array}$ & CARG & $\begin{array}{r}\text { CG } 1.0305 \\
-15.6784\end{array}$ & $\begin{array}{r}0.00 \\
-0.00\end{array}$ & 0.00 & $\begin{array}{l}10 \\
21 \\
24\end{array}$ & $\begin{array}{r}-7.65 \\
1.70 \\
5.94\end{array}$ & $\begin{array}{r}-4.69 \\
1.13 \\
3.56\end{array}$ & $\begin{array}{l}0.06 \\
0.00 \\
0.05\end{array}$ \\
\hline $\begin{array}{c}23 \\
\text { BARRA2 } 3\end{array}$ & CARG & $\begin{array}{r}\text { CG } 1.0209 \\
-15.8940\end{array}$ & $\begin{array}{l}-3.20 \\
-1.60\end{array}$ & 0.00 & $\begin{array}{l}15 \\
24\end{array}$ & $\begin{array}{r}-4.44 \\
1.24\end{array}$ & $\begin{array}{r}-2.19 \\
0.59\end{array}$ & $\begin{array}{l}0.02 \\
0.00\end{array}$ \\
\hline $\begin{array}{c}24 \\
\text { BARRA24 }\end{array}$ & CARG & $\begin{array}{r}\text { CG } 1.0177 \\
-16.0360\end{array}$ & $\begin{array}{l}-8.70 \\
-6.70\end{array}$ & 4.51 & $\begin{array}{l}22 \\
23 \\
25\end{array}$ & $\begin{array}{l}-5.89 \\
-1.24 \\
-1.57\end{array}$ & $\begin{array}{r}-3.48 \\
-0.58 \\
1.87\end{array}$ & $\begin{array}{l}0.05 \\
0.00 \\
0.01\end{array}$ \\
\hline $\begin{array}{c}25 \\
\text { BARRA2 } 5\end{array}$ & CARG & $\begin{array}{r}\text { CG } 1.0146 \\
-15.5537\end{array}$ & $\begin{array}{r}0.00 \\
-0.00\end{array}$ & 0.00 & $\begin{array}{l}24 \\
26 \\
27\end{array}$ & $\begin{array}{r}1.58 \\
3.54 \\
-5.13\end{array}$ & $\begin{array}{r}-1.85 \\
2.37 \\
-0.52\end{array}$ & $\begin{array}{l}0.01 \\
0.04 \\
0.03\end{array}$ \\
\hline $\begin{array}{c}26 \\
\text { BARRA2 } 6\end{array}$ & CARG & $\begin{array}{r}\text { CG } 0.9969 \\
-15.9757\end{array}$ & $\begin{array}{l}-3.50 \\
-2.30\end{array}$ & 0.00 & 25 & -3.50 & -2.30 & 0.04 \\
\hline $\begin{array}{c}27 \\
\text { BARRA2 } 7\end{array}$ & CARG & $\begin{array}{r}\text { CG } 1.0213 \\
-14.9934\end{array}$ & $\begin{array}{l}-0.00 \\
-0.00\end{array}$ & 0.00 & $\begin{array}{l}25 \\
28 \\
29 \\
30\end{array}$ & $\begin{array}{r}5.15 \\
-18.44 \\
6.19 \\
7.09\end{array}$ & $\begin{array}{r}0.57 \\
-3.91 \\
1.67 \\
1.66\end{array}$ & $\begin{array}{l}0.03 \\
0.00 \\
0.09 \\
0.16\end{array}$ \\
\hline $\begin{array}{c}28 \\
\text { BARRA2 } 8\end{array}$ & CARG & $\begin{array}{r}\text { CG } 1.0388 \\
-11.0473\end{array}$ & $\begin{array}{l}0.00 \\
0.00\end{array}$ & 0.00 & $\begin{array}{r}6 \\
8 \\
27\end{array}$ & $\begin{array}{r}-18.91 \\
0.47 \\
18.44\end{array}$ & $\begin{array}{r}-2.42 \\
-2.83 \\
5.25\end{array}$ & $\begin{array}{l}0.06 \\
0.00 \\
0.00\end{array}$ \\
\hline $\begin{array}{c}29 \\
\text { BARRA29 }\end{array}$ & CARG & $\begin{array}{r}\text { CG } 1.0014 \\
-16.2283\end{array}$ & $\begin{array}{l}-2.40 \\
-0.90\end{array}$ & 0.00 & $\begin{array}{l}27 \\
30\end{array}$ & $\begin{array}{r}-6.10 \\
3.70\end{array}$ & $\begin{array}{r}-1.51 \\
0.61\end{array}$ & $\begin{array}{l}0.09 \\
0.03\end{array}$ \\
\hline $\begin{array}{c}30 \\
\text { BARRA } 30\end{array}$ & CARG & $\begin{array}{r}\text { CG } 0.9899 \\
-17.1147\end{array}$ & $\begin{array}{r}-10.60 \\
-1.90\end{array}$ & 0.00 & $\begin{array}{l}27 \\
29\end{array}$ & $\begin{array}{l}-6.93 \\
-3.67\end{array}$ & $\begin{array}{l}-1.36 \\
-0.54\end{array}$ & $\begin{array}{l}0.16 \\
0.03\end{array}$ \\
\hline
\end{tabular}

PERDAS ATIVAS DO SISTEMA $=16.3564 \mathrm{~mm}$

TOTAL DE INJECAO DE REATIVO DO SISTEMA $=147.7773 \mathrm{MVAR}$

\begin{tabular}{|c|c|c|c|c|}
\hline & & MARIO & TAPS & \\
\hline NI & NE & TAPMIN & TAP & TAPMAX \\
\hline 6 & 9 & 0.9500 & 1.0000 & 1.0500 \\
\hline 6 & 10 & 0.9500 & 1.0000 & 1.0500 \\
\hline 4 & 12 & 0.9500 & 1.0000 & 1.0500 \\
\hline 28 & 27 & 0.9500 & 0.9999 & 1.0500 \\
\hline
\end{tabular}

$\begin{array}{ccccc}\begin{array}{c}\text { SUMARIO } \\ \text { barra }\end{array} & S 3 & S 4 & \text { p MULTI } & \\ 1 & 0.0003 & 0.1497 & -0.2833 & 0.0032 \\ 2 & 0.0213 & 0.1287 & -0.0226 & 0.0037 \\ 3 & 0.0418 & 0.1082 & -0.0115 & 0.0045 \\ 4 & 0.0508 & 0.0992 & -0.0095 & 0.0049 \\ 5 & 0.0599 & 0.0901 & -0.0080 & 0.0054 \\ 6 & 0.0569 & 0.0931 & -0.0085 & 0.0052 \\ 7 & 0.0661 & 0.0839 & -0.0073 & 0.0057 \\ 8 & 0.0583 & 0.0917 & -0.0083 & 0.0053 \\ 9 & 0.0483 & 0.1017 & -0.0100 & 0.0047 \\ 10 & 0.0572 & 0.0928 & -0.0084 & 0.0052 \\ 11 & 0.0202 & 0.1298 & -0.0238 & 0.0037 \\ 12 & 0.0528 & 0.0972 & -0.0091 & 0.0050 \\ 13 & 0.0284 & 0.1216 & -0.0170 & 0.0040 \\ 14 & 0.0668 & 0.0832 & -0.0072 & 0.0058 \\ 15 & 0.0704 & 0.0796 & -0.0068 & 0.0061 \\ 16 & 0.0619 & 0.0881 & -0.0078 & 0.0055 \\ 17 & 0.0640 & 0.0860 & -0.0075 & 0.0056 \\ 18 & 0.0780 & 0.0720 & -0.0062 & 0.0067 \\ 19 & 0.0793 & 0.0707 & -0.0061 & 0.0068 \\ 20 & 0.0746 & 0.0754 & -0.0065 & 0.0064\end{array}$




\begin{tabular}{|c|c|c|c|c|}
\hline 21 & 0.0699 & 0.0801 & -0.0069 & 0.0060 \\
\hline 22 & 0.0695 & 0.0805 & -0.0069 & 0.0060 \\
\hline 23 & 0.0791 & 0.0709 & -0.0061 & 0.0068 \\
\hline 24 & 0.0823 & 0.0677 & -0.0059 & 0.0071 \\
\hline 25 & 0.0854 & 0.0646 & -0.0056 & 0.0075 \\
\hline 26 & 0.1031 & 0.0469 & -0.0047 & 0.0103 \\
\hline 27 & 0.0787 & 0.0713 & -0.0061 & 0.0068 \\
\hline 28 & 0.0612 & 0.0888 & -0.0079 & 0.0054 \\
\hline 29 & 0.0986 & 0.0514 & -0.0049 & 0.0094 \\
\hline 30 & 0.1101 & 0.0399 & -0.0044 & 0.0121 \\
\hline \multicolumn{5}{|c|}{$\begin{array}{l}\text { SUMARIO DOS MULTIPLICADORES E VARIAVEIS DE FOLGA } \\
\text { DAS BARRAS COM CONTROLE DE REATIVO }\end{array}$} \\
\hline barra & s1 & s2 & pil & pi2 \\
\hline 2 & 0.0012 & 0.8988 & -0.0029 & 0.0005 \\
\hline 5 & 0.0674 & 0.7326 & -0.0060 & 0.0007 \\
\hline 8 & 0.0451 & 0.4549 & -0.0104 & 0.0011 \\
\hline 11 & 0.0945 & 0.2055 & -0.0051 & 0.0023 \\
\hline 13 & 0.0528 & 0.2472 & -0.0090 & 0.0019 \\
\hline
\end{tabular}

\section{BANCO DE SAIDA DE DADOS PARA O SISTEMA ELÉTRICO DE 118 BARRAS}

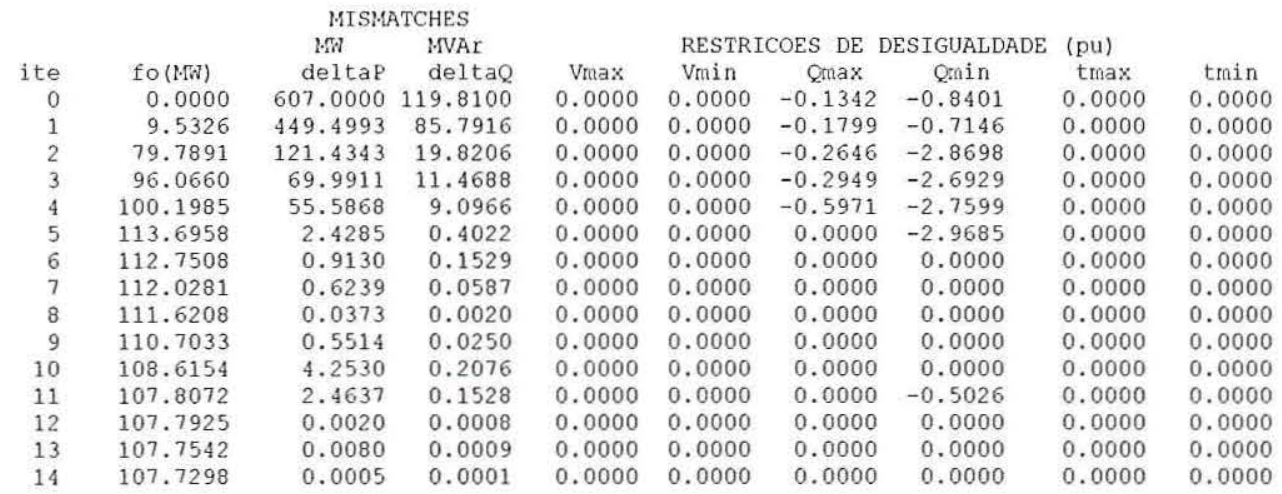

UNIVERSIDADE DE SAO PAULO - ESCOLA DE ENGENHARIA DE SAO CARLOS RELATORIO DE SAIDA - FLUXO DE CARGA OTIMO CONVERGEICIA EM 14 ITERACOES

NUMERO DE BARRAS DO SISTEMA $=118$

NUMERO DE LINHAS DO SISTEMA $=186$

NUMERO DE TAPS VARIAVEIS DO SISTEMA $=9$

NUMERO DE BARRAS CO -1 CONTROLE DE REATIVO $=51$

MATRIZ LAGRANGEANA

NUMERO DE ELEMENTOS DIFERENTES DE ZERO $=9362$

DIMENSAO DA MATRIZ $=1139 \times 1139$

SITUACAO DO PARAMETRO DE BARREIRA

parametro de barreira inicial $(V \in Q)=0.00100000$

parametro de barreira final $(V$ e $Q)=0.00000551$

parametro de beta $(\mathrm{V}$ e Q $)=1.4500$

parametro de barreira inicial (tap) $=55.00000000$

parametro de barreira final (tap) $=0.30283820$

parametro de beta $($ tap) $=1.4500$

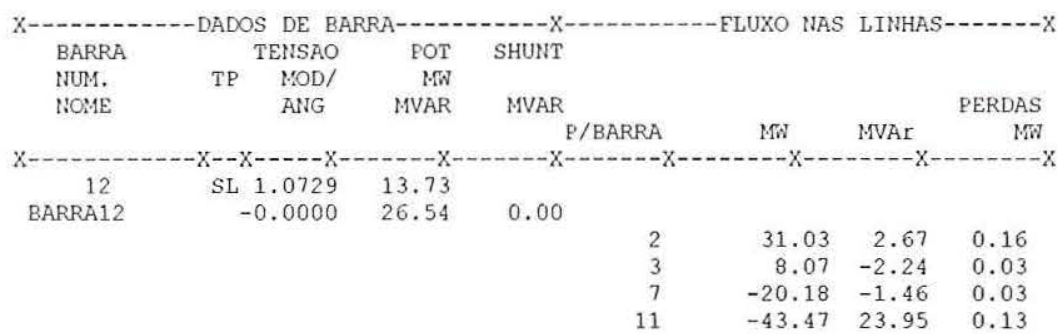




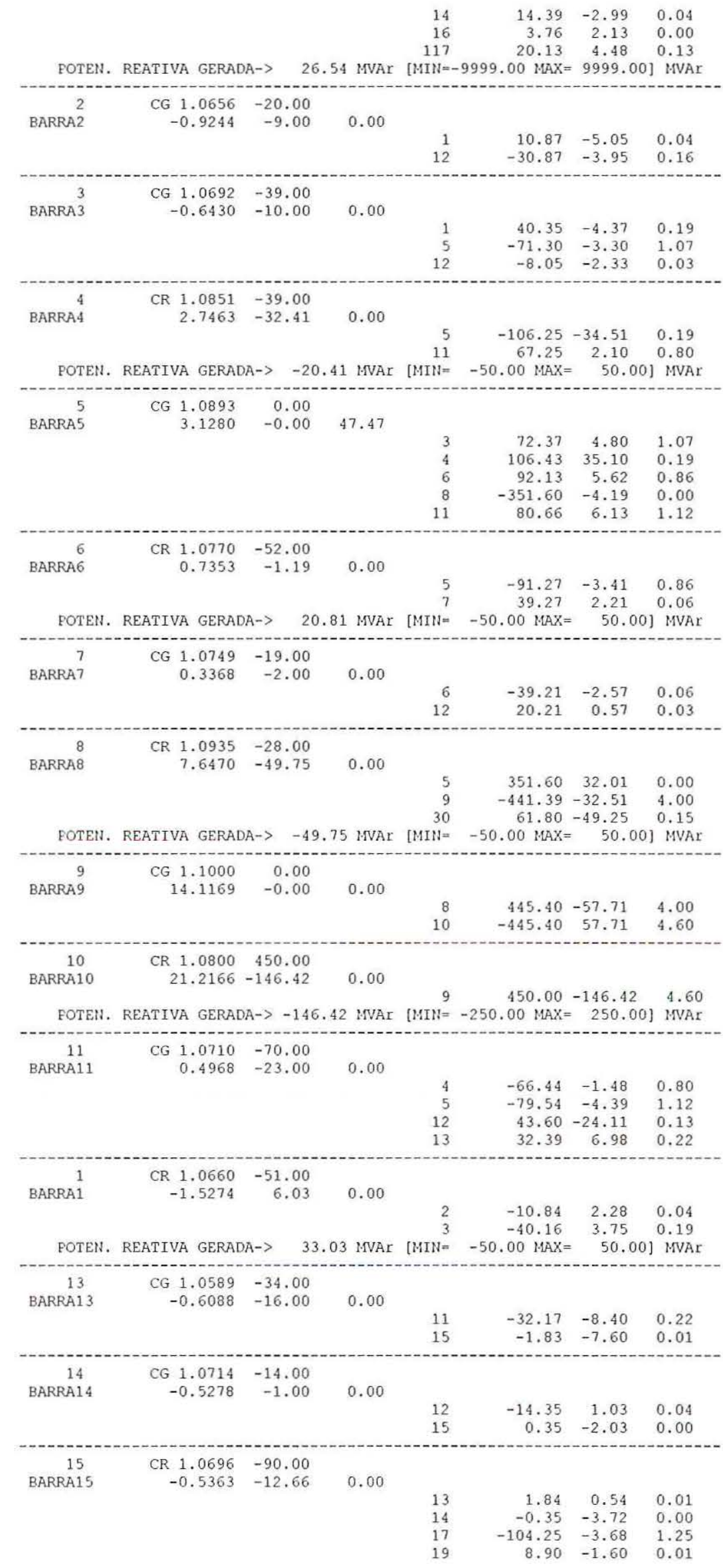




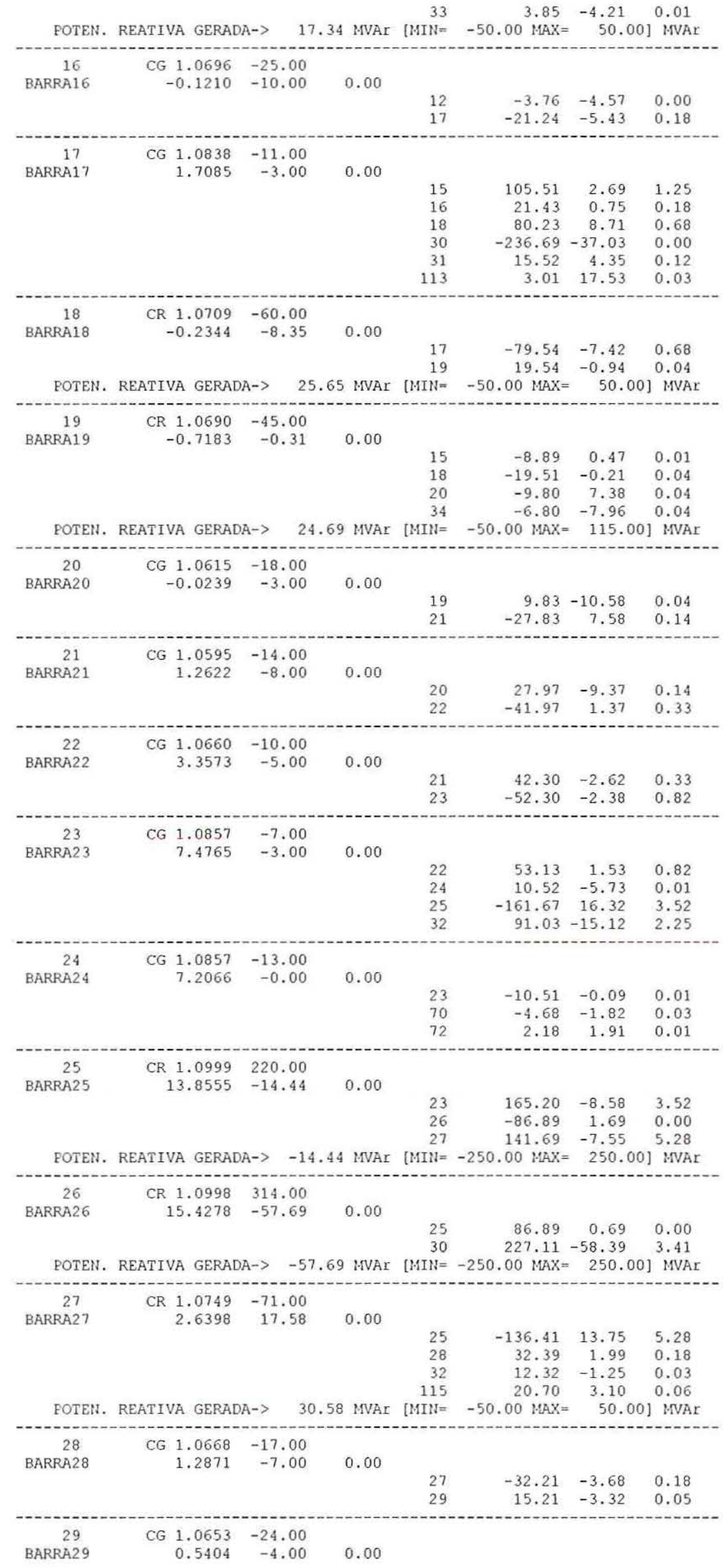




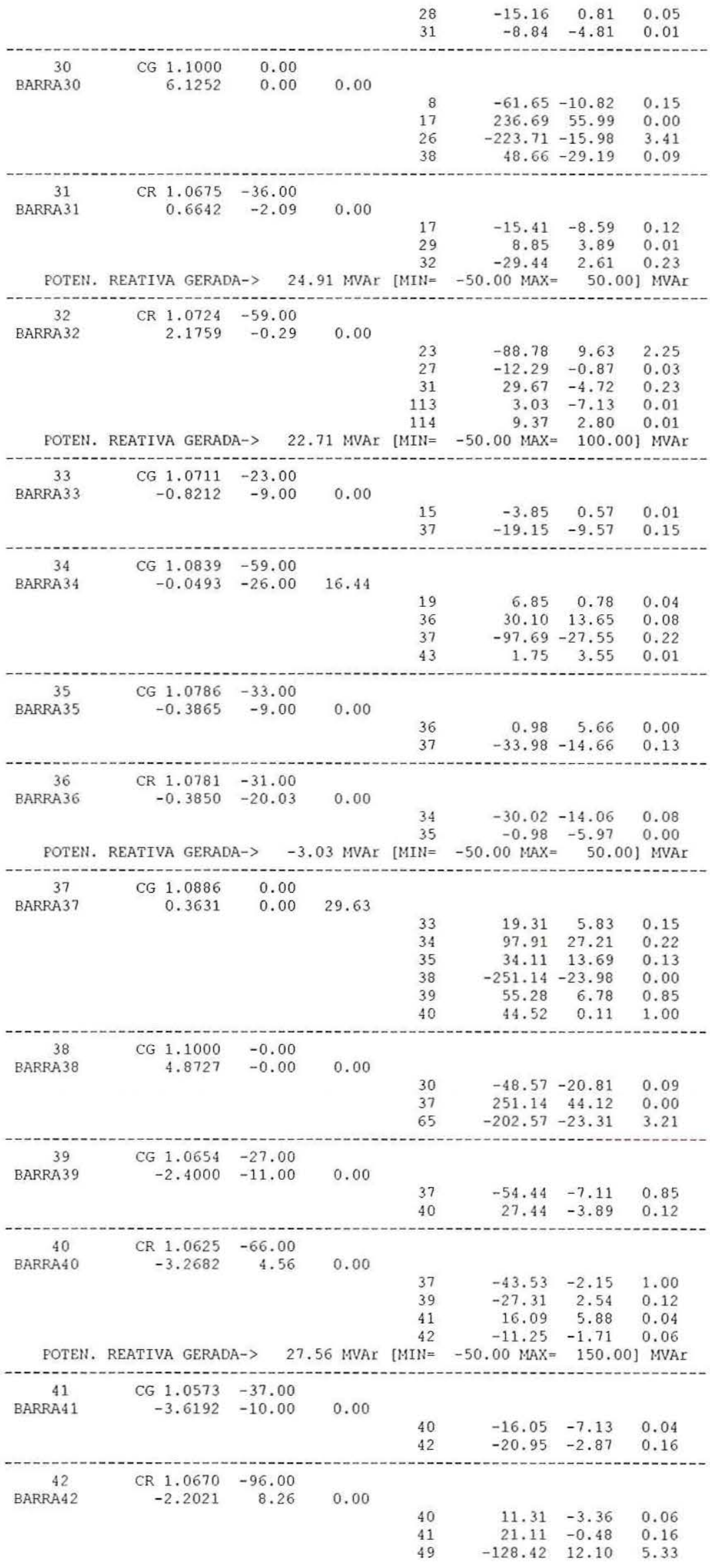




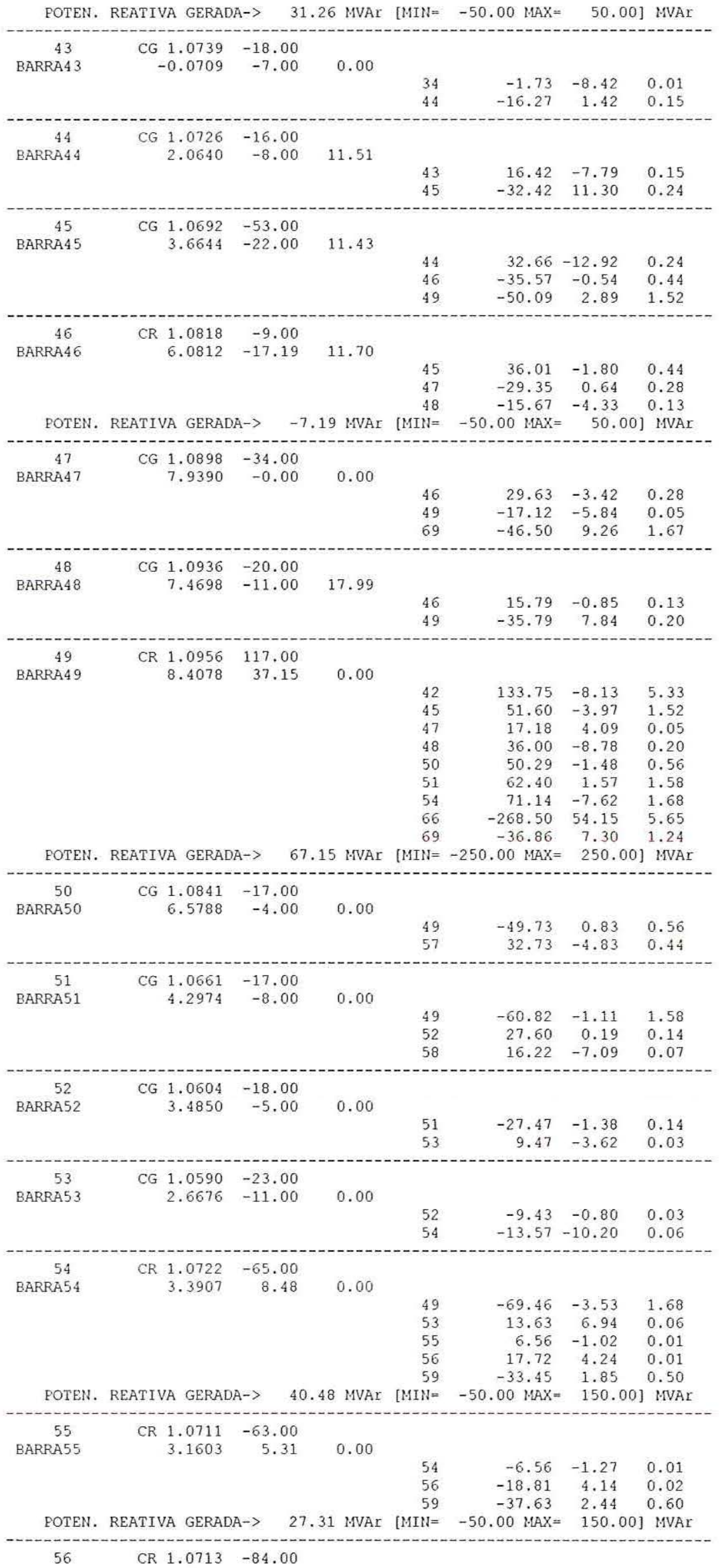




\begin{tabular}{|c|c|c|c|c|c|c|c|}
\hline 更 & REATIVA GERAD & 26.09 & 9 MVAr & $\begin{array}{r}54 \\
55 \\
57 \\
58 \\
59 \\
{[M I N=}\end{array}$ & $\begin{array}{r}-17.71 \\
18.82 \\
-20.16 \\
-4.13 \\
-60.82 \\
-50.00 \mathrm{MAX}=\end{array}$ & $\begin{aligned} &-5.06 \\
&-4.52 \\
& 2.77 \\
& 5.53 \\
&=9.36 \\
&=150.00]\end{aligned}$ & $\begin{array}{l}0.01 \\
0.02 \\
0.13 \\
0.02 \\
1.40 \\
\text { JVAr }\end{array}$ \\
\hline $\begin{array}{c}57 \\
\text { BARRA57 }\end{array}$ & $\begin{array}{r}\text { CG } 1.0742 \\
4.3536\end{array}$ & $\begin{array}{r}-12.00 \\
-3.00\end{array}$ & 0.00 & $\begin{array}{l}50 \\
56\end{array}$ & $\begin{array}{r}-32.29 \\
20.29\end{array}$ & $\begin{array}{r}2.20 \\
-5.20\end{array}$ & $\begin{array}{l}0.44 \\
0.13\end{array}$ \\
\hline $\begin{array}{c}58 \\
\text { BARRA58 }\end{array}$ & $\begin{array}{r}\text { CG } \begin{array}{r}1.0664 \\
3.6318\end{array}\end{array}$ & $\begin{array}{r}-12.00 \\
-3.00\end{array}$ & 0.00 & $\begin{array}{l}51 \\
56\end{array}$ & $\begin{array}{r}-16.15 \\
4.15\end{array}$ & $\begin{array}{r}5.24 \\
-8.24\end{array}$ & $\begin{array}{l}0.07 \\
0.02\end{array}$ \\
\hline $\begin{array}{c}59 \\
\text { BARRA59 } \\
\\
\\
\text { EOTEN. }\end{array}$ & $\begin{array}{r}\text { CR } 1.0791 \\
7.3234\end{array}$ & DA-> 49.88 & 0.00 & $\begin{array}{r}54 \\
55 \\
56 \\
60 \\
61 \\
63 \\
\text { [MIN }=\end{array}$ & $\begin{array}{r}33.95 \\
38.23 \\
62.22 \\
-45.66 \\
-54.35 \\
-156.39 \\
-50.00 \mathrm{MAX}=\end{array}$ & $\begin{aligned} &-6.48 \\
&-6.24 \\
&-17.93 \\
&-3.68 \\
&-3.44 \\
&-25.35 \\
&=\quad 50.00]\end{aligned}$ & $\begin{array}{l}0.50 \\
0.60 \\
1.40 \\
0.57 \\
0.83 \\
0.00 \\
\text { ] MVAr }\end{array}$ \\
\hline $\begin{array}{c}60 \\
\text { BARRA } 60\end{array}$ & $\begin{array}{r}\text { CG } 1.0962 \\
10.5086\end{array}$ & $\begin{array}{r}-78.00 \\
-3.00\end{array}$ & 0.00 & $\begin{array}{l}59 \\
61 \\
62\end{array}$ & $\begin{array}{r}46.22 \\
-115.77 \\
-8.45\end{array}$ & $\begin{array}{r}1.83 \\
-6.92 \\
2.09\end{array}$ & $\begin{array}{l}0.57 \\
0.30 \\
0.01\end{array}$ \\
\hline $\begin{array}{l}61 \\
\text { BARRA61 } \\
\text { POTEN. }\end{array}$ & $\begin{array}{r}\text { CR } 1.0998 \\
11.2438\end{array}$ & $\begin{array}{r}160.00 \\
38.33\end{array}$ & $\begin{array}{l}0.00 \\
\text { MVAr }\end{array}$ & $\begin{array}{r}59 \\
60 \\
62 \\
64 \\
\text { [MIN= }\end{array}$ & $\begin{array}{r}55.19 \\
116.07 \\
28.82 \\
-40.07 \\
-250.00 \text { MAX }=\end{array}$ & $\begin{aligned} & 2.64 \\
& 6.68 \\
& 5.46 \\
& 23.56 \\
&=\quad 250.001\end{aligned}$ & $\begin{array}{l}0.83 \\
0.30 \\
0.06 \\
0.00 \\
\text { ] HVAr }\end{array}$ \\
\hline $\begin{array}{l}62 \\
\text { BARRA } 62 \\
\\
\text { POTEN. }\end{array}$ & $\begin{array}{r}\text { CR } 1.0957 \\
10.7523\end{array}$ & $\begin{array}{r}-77.00 \\
-0.67\end{array}$ & $\begin{array}{l}0.00 \\
\text { MVAr }\end{array}$ & $\begin{array}{r}60 \\
61 \\
66 \\
67 \\
\text { [MIN = }\end{array}$ & $\begin{array}{r}8.46 \\
-28.76 \\
-34.93 \\
-21.77 \\
-50.00 \mathrm{MAX}=\end{array}$ & $\begin{aligned} &-3.82 \\
&-6.37 \\
& 3.29 \\
& \\
&=\quad 6.22 \\
& 50.001\end{aligned}$ & $\begin{array}{l}0.01 \\
0.06 \\
0.51 \\
0.12 \\
\text { ] MVAr }\end{array}$ \\
\hline $\begin{array}{c}63 \\
\text { BARRA63 }\end{array}$ & $\begin{array}{r}\text { CG } 1.0875 \\
10.2663\end{array}$ & $\begin{array}{l}-0.00 \\
-0.00\end{array}$ & 0.00 & $\begin{array}{l}59 \\
64\end{array}$ & $\begin{array}{r}156.39 \\
-156.39\end{array}$ & $\begin{array}{r}33.67 \\
-33.67\end{array}$ & $\begin{array}{l}0.00 \\
0.36\end{array}$ \\
\hline $\begin{array}{c}64 \\
\text { BARRA64 }\end{array}$ & $\begin{array}{r}\text { CG } 1.0942 \\
11.7552\end{array}$ & $\begin{array}{r}-0.00 \\
0.00\end{array}$ & 0.00 & $\begin{array}{l}61 \\
63 \\
65\end{array}$ & $\begin{array}{r}40.07 \\
156.76 \\
-196.83\end{array}$ & $\begin{array}{r}-23.08 \\
12.18 \\
10.90\end{array}$ & $\begin{array}{l}0.00 \\
0.36 \\
0.90\end{array}$ \\
\hline $\begin{array}{c}65 \\
\text { BARRA65 } \\
\\
\text { FOTEN. }\end{array}$ & $\begin{array}{r}\text { CR } 1.0912 \\
14.6523 \\
\text { REATIVA GERAD }\end{array}$ & $D A->-244.06$ & $\begin{array}{l}0.00 \\
\text { MVAr }\end{array}$ & $\begin{array}{r}38 \\
64 \\
66 \\
68 \\
{[\text { MIN }=}\end{array}$ & $\begin{array}{r}205.78 \\
197.73 \\
6.94 \\
-19.45 \\
-250.00 \mathrm{MAX}=\end{array}$ & $\begin{array}{l}-79.08 \\
-46.22 \\
-25.83 \\
-92.94 \\
=\quad 250.00]\end{array}$ & $\begin{array}{l}3.21 \\
0.90 \\
0.00 \\
0.04 \\
\text { ] MVAr }\end{array}$ \\
\hline $\begin{array}{c}66 \\
\text { BARRA } 66\end{array}$ & $\begin{array}{r}\text { CR } 1.1000 \\
14.5297\end{array}$ & $\begin{array}{l}353.00 \\
-16.70\end{array}$ & MVAr & $\begin{array}{r}49 \\
62 \\
65 \\
67 \\
\text { [MIM] }\end{array}$ & $\begin{array}{r}274.15 \\
35.43 \\
-6.94 \\
50.36 \\
-250.00 \mathrm{MAX}=\end{array}$ & $\begin{array}{l}-31.28 \\
-7.96 \\
26.05 \\
-3.50 \\
=250.00]\end{array}$ & $\begin{array}{l}5.65 \\
0.51 \\
0.00 \\
0.47 \\
\text { ] HVAr }\end{array}$ \\
\hline $\begin{array}{c}67 \\
\text { BARRA } 67\end{array}$ & $\begin{array}{r}\text { CG } 1.0924 \\
12.0717\end{array}$ & $\begin{array}{r}-28.00 \\
-7.00\end{array}$ & 0.00 & $\begin{array}{l}62 \\
66\end{array}$ & $\begin{array}{r}21.89 \\
-49.89\end{array}$ & $\begin{array}{r}-9.41 \\
2.41\end{array}$ & $\begin{array}{l}0.12 \\
0.47\end{array}$ \\
\hline $\begin{array}{c}68 \\
\text { BARRA68 }\end{array}$ & $\begin{array}{r}\text { CG } 1.0995 \\
14.7647\end{array}$ & $\begin{array}{r}0.00 \\
-0.00\end{array}$ & 0.00 & $\begin{array}{l}65 \\
69 \\
81\end{array}$ & $\begin{array}{r}19.49 \\
4.89 \\
-24.39\end{array}$ & $\begin{array}{r}16.85 \\
2.49 \\
-49.40\end{array}$ & $\begin{array}{l}0.04 \\
0.00 \\
0.01\end{array}$ \\
\hline
\end{tabular}




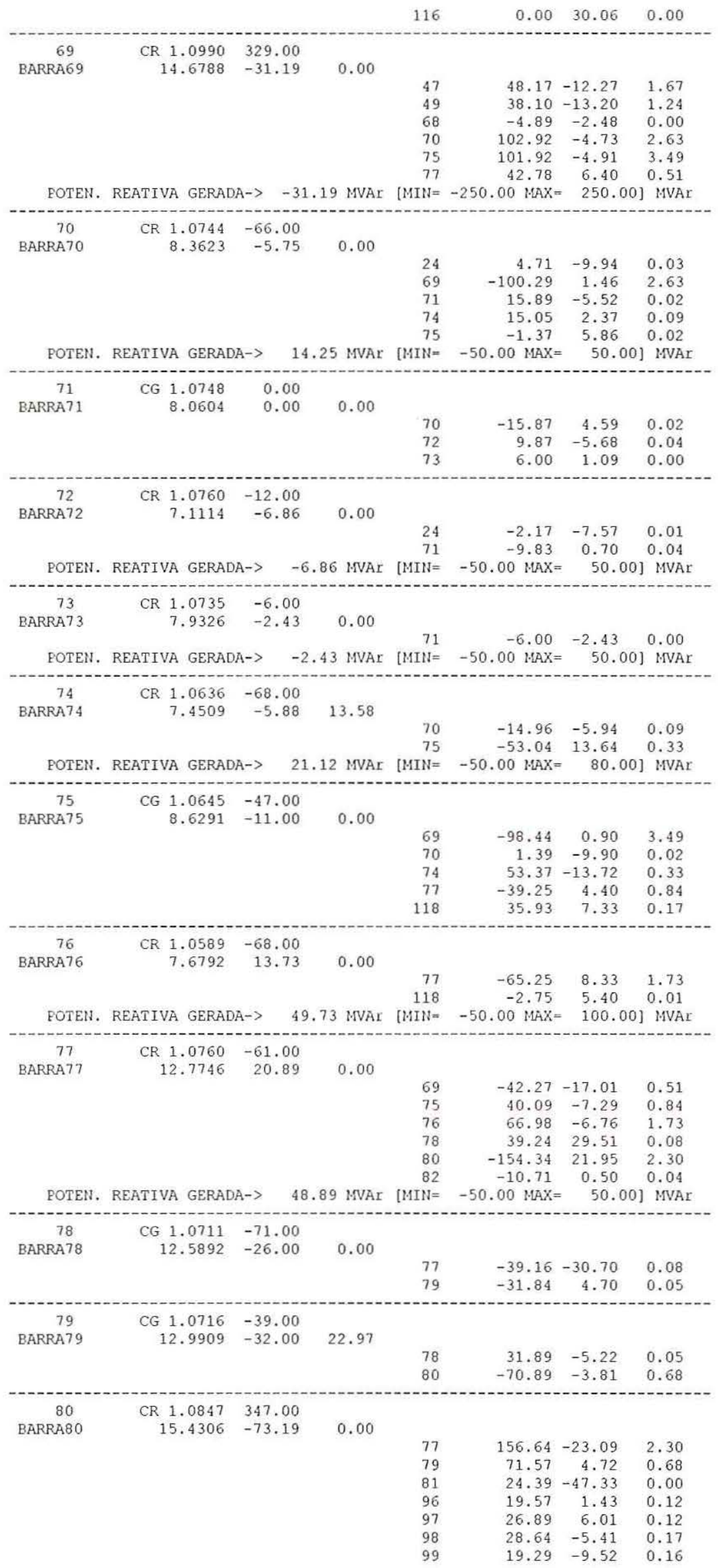


POTEN. REATIVA GERADA $\rightarrow-47.19$ MVAr [MIN $=-250.00$ MAX $=250.00]$ MVAr

\begin{tabular}{|c|c|c|c|c|c|c|c|}
\hline \multirow{4}{*}{$\begin{array}{c}81 \\
\text { BARRA81 }\end{array}$} & CG 1.1000 & \multirow{4}{*}{$\begin{array}{l}-0.00 \\
-0.00\end{array}$} & \multirow{4}{*}{0.00} & \multirow{4}{*}{$\begin{array}{l}68 \\
80\end{array}$} & \multirow{4}{*}{$\begin{array}{r}24.39 \\
-24.39\end{array}$} & \multirow{4}{*}{$\begin{array}{r}-48.22 \\
48.22\end{array}$} & \multirow{4}{*}{$\begin{array}{l}0.01 \\
0.00\end{array}$} \\
\hline & 14.9976 & & & & & & \\
\hline & & & & & & & \\
\hline & & & & & & & \\
\hline 82 & CG 1.0748 & -54.00 & & & & & \\
\hline \multirow[t]{4}{*}{ BARRA82 } & 13.3043 & -27.00 & 23.10 & & & & \\
\hline & & & & 77 & 10.74 & -9.85 & 0.04 \\
\hline & & & & 83 & -51.20 & -1.91 & 0.25 \\
\hline & & & & 96 & -13.54 & 7.86 & 0.04 \\
\hline 83 & CG 1.0802 & -20.00 & & & & & \\
\hline \multirow[t]{4}{*}{ BARRA 83} & 14.2320 & -10.00 & 11.67 & & & & \\
\hline & & & & 82 & 51.45 & -1.67 & 0.25 \\
\hline & & & & 84 & -27.32 & 5.07 & 0.42 \\
\hline & & & & 85 & -44.14 & -1.74 & 0.72 \\
\hline 84 & CG 1.0886 & -11.00 & & & & & \\
\hline \multirow[t]{3}{*}{ BARRA 84} & 16.1895 & -7.00 & 0.00 & & & & \\
\hline & & & & 83 & 27.74 & -7.21 & 0.42 \\
\hline & & & & 85 & -38.74 & 0.21 & 0.38 \\
\hline 85 & CR 1.0990 & -24.00 & & & & & \\
\hline \multirow{6}{*}{ BARRA8 5} & 17.3924 & 29.59 & 0.00 & & & & \\
\hline & & & & 83 & 44.86 & 0.08 & 0.72 \\
\hline & & & & 84 & 39.12 & -0.88 & 0.38 \\
\hline & & & & 86 & 17.09 & 1.78 & 0.09 \\
\hline & & & & 88 & -51.73 & 17.85 & 0.51 \\
\hline & & & & 89 & -73.34 & 10.75 & 1.10 \\
\hline & & & 59 MVAr & IN $=$ & $.00 \mathrm{MAX}=$ & $=80$. & IVA \\
\hline
\end{tabular}

POTEN. REATIVA GERADA-> 44.59 MVAr [MIN $=-50.00 \mathrm{MAX}=80.00]$ MVAr

\begin{tabular}{|c|c|c|c|c|c|c|c|}
\hline $\begin{array}{c}86 \\
\text { BARRA86 }\end{array}$ & $\begin{array}{r}\text { CG } 1.0899 \\
16.4445\end{array}$ & $\begin{array}{l}-21.00 \\
-10.00\end{array}$ & 0.00 & $\begin{array}{l}85 \\
87\end{array}$ & $\begin{array}{r}-17.01 \\
-3.99\end{array}$ & $\begin{array}{l}-4.78 \\
-5.22\end{array}$ & $\begin{array}{l}0.09 \\
0.01\end{array}$ \\
\hline $\begin{array}{c}87 \\
\text { BARRA87 }\end{array}$ & $\begin{array}{r}\text { CR } 1.0958 \\
16.8069\end{array}$ & $\begin{array}{r}4.00 \\
-0.05\end{array}$ & 0.00 & 86 & 4.00 & -0.05 & 0.01 \\
\hline
\end{tabular}

POTEN. REATIVA GERADA $\rightarrow \quad-0.05$ MVAr [MIN $=-250.00 \mathrm{MAX}=250.00$ ]

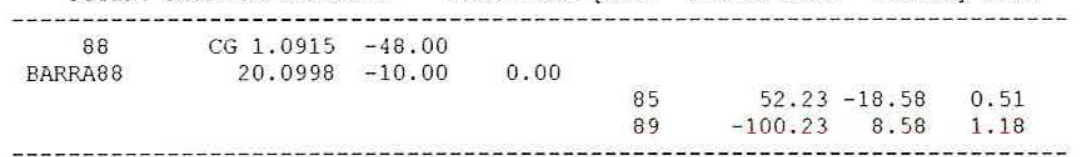

\begin{tabular}{|c|c|c|c|c|c|c|c|}
\hline 89 & CR 1.1000 & 607.00 & & & & & \\
\hline \multirow[t]{5}{*}{ BARRA 69} & 23.5721 & -74.29 & 0.00 & & & & \\
\hline & & & & 85 & 74.45 & -8.46 & 1.10 \\
\hline & & & & 88 & 101.42 & -4.84 & 1.18 \\
\hline & & & & 90 & 167.41 & -16.08 & 3.80 \\
\hline & & & & 92 & 263.73 & -44.90 & 4.69 \\
\hline
\end{tabular}

POTEN. REATIVA GERADA-> -74.29 MVAr [MIN $=-250.00 \mathrm{MAX}=250.00$ ] HVAr

\begin{tabular}{|c|c|c|c|c|c|c|c|}
\hline \multirow{4}{*}{$\begin{array}{c}90 \\
\text { BARRA90 }\end{array}$} & \multirow{4}{*}{$\begin{array}{r}\text { CR } 1.0835 \\
18.2688\end{array}$} & \multicolumn{2}{|l|}{-163.00} & \multirow[b]{3}{*}{89} & \multirow[b]{3}{*}{-163.61} & \multirow[b]{3}{*}{12.27} & \multirow[b]{3}{*}{3.80} \\
\hline & & 4.98 & 0.00 & & & & \\
\hline & & & & & & & \\
\hline & & & & 91 & 0.61 & -7.29 & 0.01 \\
\hline FOTEN. & REATIVA GERAD & 46.98 & 3 MVAr & {$[\mathrm{MIN}=$} & $-50.00 \mathrm{MAX}=$ & $100.00]$ & JVAr \\
\hline \multirow{4}{*}{$\begin{array}{c}91 \\
\text { BARRA91 }\end{array}$} & CR 1.0880 & -10.00 & & & & & \\
\hline & 18.1694 & -3.22 & 0.00 & & & & \\
\hline & & & & 90 & -0.61 & 4.79 & 0.01 \\
\hline & & & & 92 & -9.39 & -8.01 & 0.04 \\
\hline POTEN. & REATIVA GERAD & -3.22 & 2 MVAY & {$[\mathrm{MIN}=$} & $-50.00 \mathrm{MAX}=$ & $50.00]$ & ] MVAr \\
\hline 92 & CR 1.0985 & -65.00 & & & & & \\
\hline \multirow[t]{7}{*}{ BARRA92 } & 18.6295 & 48.59 & 0.00 & & & & \\
\hline & & & & 89 & -259.03 & 55.77 & 4.69 \\
\hline & & & & 91 & 9.44 & 4.24 & 0.04 \\
\hline & & & & 93 & 57.95 & 1.09 & 0.72 \\
\hline & & & & 94 & 52.41 & -2.44 & 1.09 \\
\hline & & & & 100 & 30.42 & -9.87 & 0.51 \\
\hline & & & & 102 & 43.82 & -0.20 & 0.20 \\
\hline POTEN. & REATIVA GERAD & 58.59 & 9 HVAr & {$[\mathrm{MIN}=$} & $-50.00 \mathrm{MAX}=$ & $100.00]$ & ] MVAr \\
\hline 93 & CG 1.0840 & -12.00 & & & & & \\
\hline \multirow{3}{*}{ BARRA93 } & 16.2941 & -7.00 & 0.00 & & & & \\
\hline & & & & 92 & -57.23 & -1.32 & 0.72 \\
\hline & & & & 94 & 45.23 & -5.68 & 0.39 \\
\hline \multirow{4}{*}{$\begin{array}{c}94 \\
\text { BARRA } 94\end{array}$} & CG 1.0782 & -30.00 & & & & & \\
\hline & 14.6207 & -16.00 & 0.00 & & & & \\
\hline & & & & 92 & -51.31 & 1.23 & 1.09 \\
\hline & & & & 93 & -44.84 & 4.77 & 0.39 \\
\hline
\end{tabular}




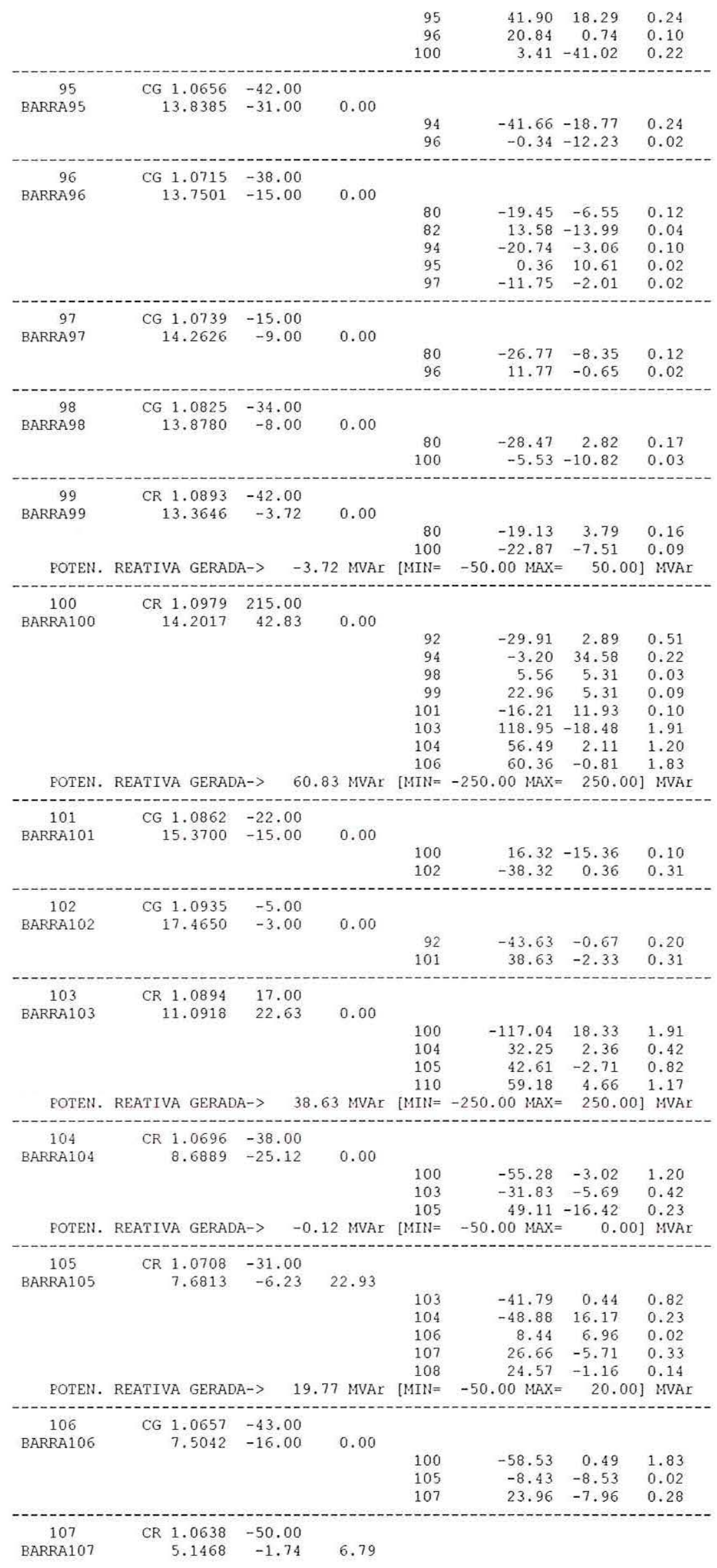




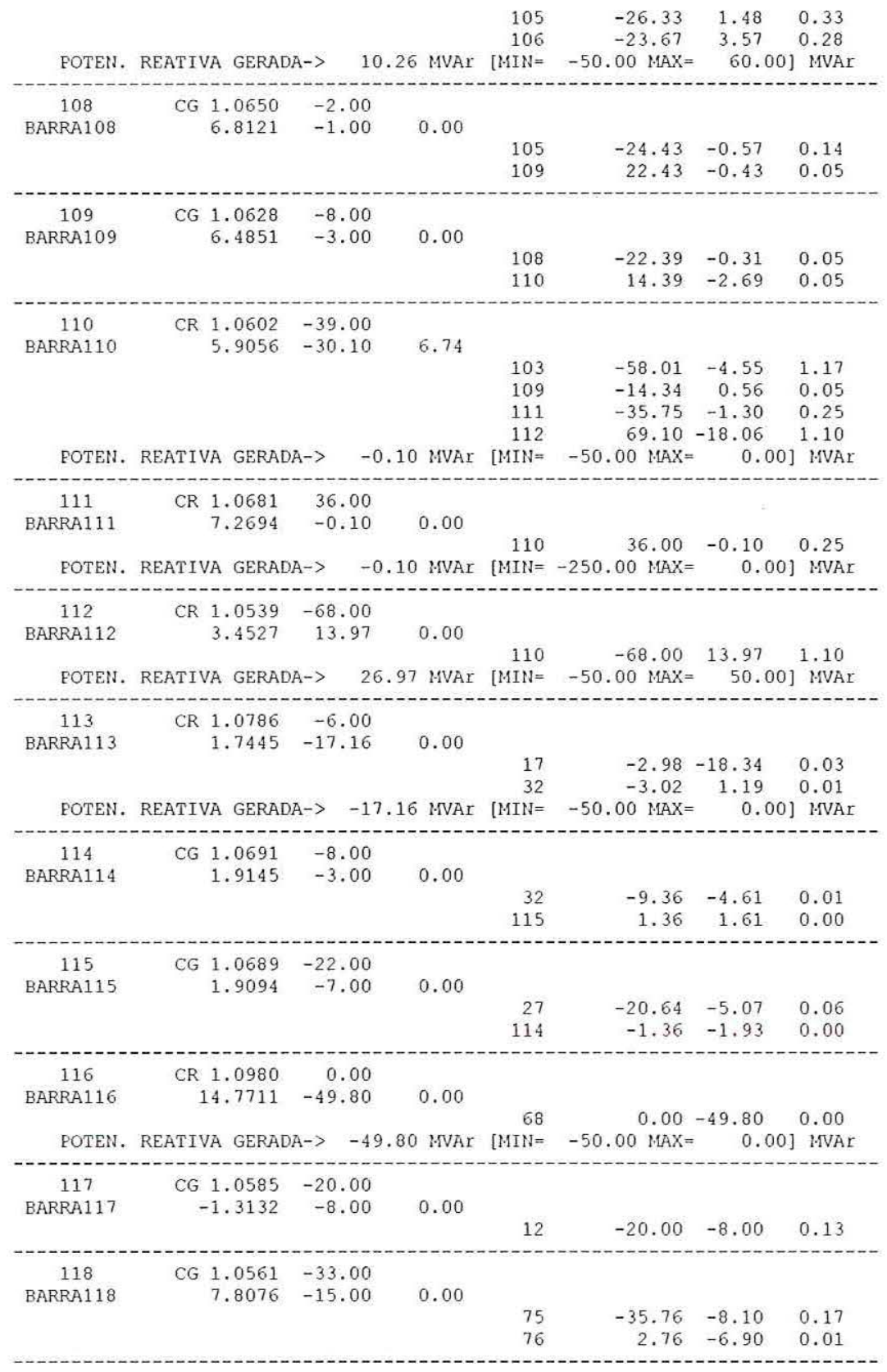

PERDAS ATIVAS DO SISTEMA $=107.7298 \mathrm{~m}$

TOTAL DE INJECAO DE REATIVO DO SISTEMA $=210.3282 \mathrm{MVAR}$

$\begin{array}{ccccc}\text { NI } & \text { NF } & \text { SUMARIO DOS TAPS } \\ 8 & 5 & 0.9500 & 0.9997 & 1.0500 \\ 26 & 25 & 0.9500 & 1.0000 & 1.0500 \\ 30 & 17 & 0.9500 & 0.9997 & 1.0500 \\ 38 & 37 & 0.9500 & 0.9997 & 1.0500 \\ 63 & 59 & 0.9500 & 0.9981 & 1.0500 \\ 64 & 61 & 0.9500 & 1.0001 & 1.0500 \\ 65 & 66 & 0.9500 & 1.0000 & 1.0500 \\ 68 & 69 & 0.9500 & 0.9997 & 1.0500 \\ 81 & 80 & 0.9500 & 0.9992 & 1.0500\end{array}$

SUMARIO DOS MULTIPLICADORES E VARIAVEIS DE FOLGA DAS TENSOES

$\begin{array}{ccccc}\text { barra } & s 3 & s 4 & \text { pi } & \text { pi4 } \\ 1 & 0.0271 & 0.1229 & -0.0003 & 0.0001 \\ 2 & 0.0344 & 0.1156 & -0.0002 & 0.0001 \\ 3 & 0.0308 & 0.1192 & -0.0003 & 0.0001 \\ 4 & 0.0149 & 0.1351 & -0.0005 & 0.0001 \\ 5 & 0.0107 & 0.1393 & -0.0007 & 0.0001 \\ 6 & 0.0230 & 0.1270 & -0.0003 & 0.0001\end{array}$




\begin{tabular}{|c|c|c|c|}
\hline 0.0251 & 0.1249 & -0.0003 & 0.0001 \\
\hline 0.0065 & 0.1435 & -0.0012 & 0.0001 \\
\hline 0.0000 & 0.1500 & -0.1913 & 0.0001 \\
\hline 0.0200 & 0.1300 & -0.0004 & 0.0001 \\
\hline 0.0290 & 0.1210 & -0.0003 & 0.0001 \\
\hline 0.0340 & 0.1160 & -0.0002 & 0.0001 \\
\hline 0.0411 & 0.1089 & -0.0002 & 0.0001 \\
\hline 0.0286 & 0.1214 & -0.0003 & 0.0001 \\
\hline 0.0304 & 0.1196 & -0.0003 & 0.0001 \\
\hline 0.0304 & 0.1196 & -0.0003 & 0.0001 \\
\hline 0.0162 & 0.1338 & -0.0005 & 0.0001 \\
\hline 0.0291 & 0.1209 & -0.0003 & 0.0001 \\
\hline 0.0310 & 0.1190 & -0.0003 & 0.0001 \\
\hline 0.0385 & 0.1115 & -0.0002 & 0.0001 \\
\hline 0.0405 & 0.1095 & -0.0002 & 0.0001 \\
\hline 0.0340 & 0.1160 & -0.0002 & 0.0001 \\
\hline 0.0143 & 0.1357 & -0.0006 & 0.0001 \\
\hline 0.0143 & 0.1357 & -0.0006 & 0.0001 \\
\hline 0.0001 & 0.1499 & -0.1194 & 0.0001 \\
\hline 0.0002 & 0.1498 & -0.0321 & 0.0001 \\
\hline 0.0251 & 0.1249 & -0.0003 & 0.0001 \\
\hline 0.0332 & 0.1168 & -0.0002 & 0.0001 \\
\hline 0.0347 & 0.1153 & -0.0002 & 0.0001 \\
\hline 0.0000 & 0.1500 & -0.1885 & 0.0001 \\
\hline 0.0325 & 0.1175 & -0.0002 & 0.0001 \\
\hline 0.0276 & 0.1224 & -0.0003 & 0.0001 \\
\hline 0.0289 & 0.1211 & -0.0003 & 0.0001 \\
\hline 0.0161 & 0.1339 & -0.0005 & 0.0001 \\
\hline 0.0214 & 0.1286 & -0.0004 & 0.0001 \\
\hline 0.0219 & 0.1281 & -0.0004 & 0.0001 \\
\hline 0.0114 & 0.1386 & -0.0007 & 0.0001 \\
\hline 0.0000 & 0.1500 & -0.1303 & 0.0001 \\
\hline 0.0346 & 0.1154 & -0.0002 & 0.0001 \\
\hline 0.0375 & 0.1125 & -0.0002 & 0.0001 \\
\hline 0.0427 & 0.1073 & -0.0002 & 0.0001 \\
\hline 0.0330 & 0.1170 & -0.0002 & 0.0001 \\
\hline 0.0261 & 0.1239 & -0.0003 & 0.0001 \\
\hline 0.0274 & 0.1226 & -0.0003 & 0.0001 \\
\hline 0.0308 & 0.1192 & -0.0003 & 0.0001 \\
\hline 0.0182 & 0.1318 & -0.0004 & 0.0001 \\
\hline 0.0102 & 0.1398 & -0.0008 & 0.0001 \\
\hline 0.0064 & 0.1436 & -0.0012 & 0.0001 \\
\hline 0.0044 & 0.1456 & -0.0018 & 0.0001 \\
\hline 0.0159 & 0.1341 & -0.0005 & 0.0001 \\
\hline 0.0339 & 0.1161 & -0.0002 & 0.0001 \\
\hline 0.0396 & 0.1104 & -0.0002 & 0.0001 \\
\hline 0.0410 & 0.1090 & -0.0002 & 0.0001 \\
\hline 0.0278 & 0.1222 & -0.0003 & 0.0001 \\
\hline 0.0289 & 0.1211 & -0.0003 & 0.0001 \\
\hline 0.0287 & 0.1213 & -0.0003 & 0.0001 \\
\hline 0.0258 & 0.1242 & -0.0003 & 0.0001 \\
\hline 0.0336 & 0.1164 & -0.0002 & 0.0001 \\
\hline 0.0209 & 0.1291 & -0.0004 & 0.0001 \\
\hline 0.0038 & 0.1462 & -0.0021 & 0.0001 \\
\hline 0.0002 & 0.1498 & -0.0326 & 0.0001 \\
\hline 0.0043 & 0.1457 & -0.0018 & 0.0001 \\
\hline 0.0125 & 0.1375 & -0.0006 & 0.0001 \\
\hline 0.0058 & 0.1442 & -0.0014 & 0.0001 \\
\hline 0.0088 & 0.1412 & -0.0009 & 0.0001 \\
\hline 0.0000 & 0.1500 & -0.1927 & 0.0001 \\
\hline 0.0076 & 0.1424 & -0.0011 & 0.0001 \\
\hline 0.0005 & 0.1495 & -0.0155 & 0.0001 \\
\hline 0.0010 & 0.1490 & -0.0073 & 0.0001 \\
\hline 0.0256 & 0.1244 & -0.0003 & 0.0001 \\
\hline 0.0252 & 0.1248 & -0.0003 & 0.0001 \\
\hline 0.0240 & 0.1260 & -0.0003 & 0.0001 \\
\hline 0.0265 & 0.1235 & -0.0003 & 0.0001 \\
\hline 0.0364 & 0.1136 & -0.0002 & 0.0001 \\
\hline 0.0355 & 0.1145 & -0.0002 & 0.0001 \\
\hline 0.0411 & 0.1089 & -0.0002 & 0.0001 \\
\hline 0.0240 & 0.1260 & -0.0003 & 0.0001 \\
\hline 0.0289 & 0.1211 & -0.0003 & 0.0001 \\
\hline 0.0284 & 0.1216 & -0.0003 & 0.0001 \\
\hline 0.0153 & 0.1347 & -0.0005 & 0.0001 \\
\hline 0.0000 & 0.1500 & -0.5149 & 0.0001 \\
\hline 0.0252 & 0.1248 & -0.0003 & 0.0001 \\
\hline 0.0198 & 0.1302 & -0.0004 & 0.0001 \\
\hline 0.0114 & 0.1386 & -0.0007 & 0.0001 \\
\hline 0.0010 & 0.1490 & -0.0073 & 0.0001 \\
\hline 0.0101 & 0.1399 & -0.0008 & 0.0001 \\
\hline 0.0042 & 0.1458 & -0.0018 & 0.0001 \\
\hline 0.0085 & 0.1415 & -0.0009 & 0.0001 \\
\hline 0.0000 & 0.1500 & -0.3850 & 0.0001 \\
\hline 0.0165 & 0.1335 & -0.0005 & 0.0001 \\
\hline 0.0120 & 0.1380 & -0.0007 & 0.0001 \\
\hline 0.0015 & 0.1485 & -0.0052 & 0.0001 \\
\hline 0.0160 & 0.1340 & -0.0005 & 0.0001 \\
\hline
\end{tabular}




$\begin{array}{rllll}94 & 0.0218 & 0.1282 & -0.0004 & 0.0001 \\ 95 & 0.0344 & 0.1156 & -0.0002 & 0.0001 \\ 96 & 0.0285 & 0.1215 & -0.0003 & 0.0001 \\ 97 & 0.0261 & 0.1239 & -0.0003 & 0.0001 \\ 98 & 0.0175 & 0.1325 & -0.0005 & 0.0001 \\ 99 & 0.0107 & 0.1393 & -0.0007 & 0.0001 \\ 100 & 0.0021 & 0.1479 & -0.0035 & 0.0001 \\ 101 & 0.0138 & 0.1362 & -0.0006 & 0.0001 \\ 102 & 0.0065 & 0.1435 & -0.0012 & 0.0001 \\ 103 & 0.0106 & 0.1394 & -0.0008 & 0.0001 \\ 104 & 0.0304 & 0.1196 & -0.0003 & 0.0001 \\ 105 & 0.0292 & 0.1208 & -0.0003 & 0.0001 \\ 106 & 0.0343 & 0.1157 & -0.0002 & 0.0001 \\ 107 & 0.0362 & 0.1138 & -0.0002 & 0.0001 \\ 108 & 0.0350 & 0.1150 & -0.0002 & 0.0001 \\ 109 & 0.0372 & 0.1128 & -0.0002 & 0.0001 \\ 110 & 0.0398 & 0.1102 & -0.0002 & 0.0001 \\ 111 & 0.0319 & 0.1181 & -0.0003 & 0.0001 \\ 112 & 0.0461 & 0.1039 & -0.0002 & 0.0001 \\ 113 & 0.0214 & 0.1286 & -0.0004 & 0.0001 \\ 114 & 0.0309 & 0.1191 & -0.0003 & 0.0001 \\ 115 & 0.0311 & 0.1189 & -0.0003 & 0.0001 \\ 116 & 0.0020 & 0.1480 & -0.0040 & 0.0001 \\ 117 & 0.0415 & 0.1085 & -0.0002 & 0.0001 \\ 118 & 0.0439 & 0.1061 & -0.0002 & 0.0001\end{array}$

SUMARIO DOS MULTIPLICADORES E VARIAVEIS DE FOLGA DAS BARRAS COM CONTROLE DE REATIVO

\begin{tabular}{|c|c|c|c|c|}
\hline barra & s1 & s2 & pil & pi2 \\
\hline 4 & 0.7041 & 0.2959 & -0.0000 & 0.0000 \\
\hline 6 & 0.2919 & 0.7081 & -0.0000 & 0.0000 \\
\hline 8 & 0.9975 & 0.0025 & -0.0000 & 0.0032 \\
\hline 10 & 3.9642 & 1.0358 & -0.0000 & 0.0000 \\
\hline 12 & 0.1697 & 0.8303 & -0.0000 & 0.0000 \\
\hline 15 & 0.3266 & 0.6734 & -0.0000 & 0.0000 \\
\hline 18 & 0.2435 & 0.7565 & -0.0000 & 0.0000 \\
\hline 19 & 0.9031 & 0.7469 & -0.0000 & 0.0000 \\
\hline 25 & 2.6444 & 2.3556 & -0.0000 & 0.0000 \\
\hline 26 & 3.0769 & 1.9231 & -0.0000 & 0.0000 \\
\hline 27 & 0.1942 & 0.8058 & -0.0000 & 0.0000 \\
\hline 31 & 0.2509 & 0.7491 & -0.0000 & 0.0000 \\
\hline 32 & 0.7729 & 0.7271 & -0.0000 & 0.0000 \\
\hline 36 & 0.5303 & 0.4697 & -0.0000 & 0.0000 \\
\hline 40 & 1.2244 & 0.7756 & -0.0000 & 0.0000 \\
\hline 42 & 0.1874 & 0.8126 & -0.0000 & 0.0000 \\
\hline 46 & 0.5719 & 0.4281 & -0.0000 & 0.0000 \\
\hline 49 & 1.8285 & 3.1715 & -0.0000 & 0.0000 \\
\hline 54 & 1.0952 & 0.9048 & -0.0000 & 0.0000 \\
\hline 55 & 1.2269 & 0.7731 & -0.0000 & 0.0000 \\
\hline 56 & 1.2391 & 0.7609 & -0.0000 & 0.0000 \\
\hline 59 & 0.0012 & 0.9988 & -0.0054 & 0.0000 \\
\hline 61 & 2.1167 & 2.8833 & -0.0000 & 0.0000 \\
\hline 62 & 0.3667 & 0.6333 & -0.0000 & 0.0000 \\
\hline 65 & 4.9406 & 0.0594 & -0.0000 & 0.0001 \\
\hline 66 & 2.4870 & 2.5130 & -0.0000 & 0.0000 \\
\hline 69 & 2.8119 & 2.1881 & -0.0000 & 0.0000 \\
\hline 70 & 0.3575 & 0.6425 & -0.0000 & 0.0000 \\
\hline 72 & 0.5686 & 0.4314 & -0.0000 & 0.0000 \\
\hline 73 & 0.5243 & 0.4757 & -0.0000 & 0.0000 \\
\hline 74 & 0.5888 & 0.7112 & -0.0000 & 0.0000 \\
\hline 76 & 0.5027 & 0.9973 & -0.0000 & 0.0000 \\
\hline 77 & 0.0111 & 0.9889 & -0.0007 & 0.0000 \\
\hline 80 & 2.9719 & 2.0281 & -0.0000 & 0.0000 \\
\hline 85 & 0.3541 & 0.9459 & -0.0000 & 0.0000 \\
\hline 87 & 2.5005 & 2.4995 & -0.0000 & 0.0000 \\
\hline 89 & 3.2429 & 1.7571 & -0.0000 & 0.0000 \\
\hline 90 & 0.5302 & 0.9698 & -0.0000 & 0.0000 \\
\hline 91 & 0.5322 & 0.4678 & -0.0000 & 0.0000 \\
\hline 92 & 0.4141 & 1.0859 & -0.0000 & 0.0000 \\
\hline 99 & 0.5372 & 0.4628 & -0.0000 & 0.0000 \\
\hline 100 & 1.8917 & 3.1083 & -0.0000 & 0.0000 \\
\hline 103 & 2.1137 & 2.8863 & -0.0000 & 0.0000 \\
\hline 104 & 0.0012 & 0.4988 & -0.0054 & 0.0000 \\
\hline 105 & 0.0023 & 0.6977 & -0.0030 & 0.0000 \\
\hline 107 & 0.4974 & 0.6026 & -0.0000 & 0.0000 \\
\hline 110 & 0.0010 & 0.4990 & -0.0067 & 0.0000 \\
\hline 111 & 0.0010 & 2.4990 & -0.0066 & 0.0000 \\
\hline 112 & 0.2303 & 0.7697 & -0.0000 & 0.0000 \\
\hline 113 & 0.1716 & 0.3284 & -0.0001 & 0.0000 \\
\hline 116 & 0.4980 & 0.0020 & -0.0000 & 0.0035 \\
\hline
\end{tabular}

